\title{
GREAT MICROWAVE BURSTS AND HARD X RAYS FROM SOLAR FLARES
}

Herbert J. Wiehl, David A. Batchelor, Carol Jo Crannell, Brian R. Dennis and Phillip N. Price

JUNE 1983

National Aeronautics and Space Adminıstration

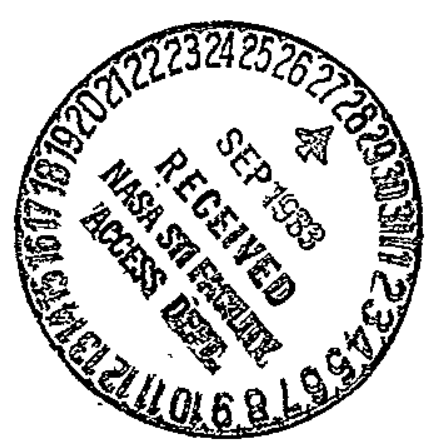

Goddard Space Flight Center Greenbelt, Maryland 2077.1 
Great Microwave Bursts and Hard X Rays from Solar Flares

\author{
Herbert J. Wieh1*, David A. Batchelor ${ }^{\dagger}$, Carol Jo Crannell, Brian R. Dennis \\ and Phillip N. Price
}

NASA Goddard Space Flight Center Laboratory for Astronomy and Solar Physics

*Swiss National Science Foundation Fellow from the University of Berne. †Also Energy/Environmental Research Group, Incorporated, Tucson, Arizona, and Department of Physics \& Astronomy, University of North Carolina, Chapel Hill. 
The microwave and hard X-ray characteristics of 13 solar flares that produced. microwave fluxes greater than 500 Solar Flux Units have been analyzed. These Great Microwave Bursts were observed in the frequency range from 3 to $35 \mathrm{GHz}$ at Berne, and simultaneous hard X-ray observations were made in the energy range from 30 to $500 \mathrm{keV}$ with the Hard X-Ray Burst Spectrometer on the Solar Maximum Mission spacecraft. The principal aim of this analysis is to determine whether or not the same distribution of energetic electrons can explain both emissions. The temporal behaviors of the microwaves as a function of frequency and the $X$ rays as a function of energy were tested for correlations, with results suggesting that high-frequency ( $>5 \mathrm{GHz}$ ) microwaves usually come. from the same electrons that produce the X-rays, while low-frequency microwaves do not. Other properties of the two emissions were found to be correlated, such as the microwave peak frequency and the $\mathrm{X}$-ray spectral index. A singletemperature and a multi-temperature model from the literature were tested for consistency with the coincident X-ray and microwave spectra at microwave burst maximum. A source area derived on the basis of the single-temperature model agrees to within the uncertainties with the observed area of the one burst for which spatially resolved X-ray images are available. Four events are inconsistent with both of the models tested, and neither of the models attempts to explain the high-frequency part of the microwave spectrum. We propose a modeI in which the emissions above and below the peak frequency originate in two different parts of a diverging magnetic loop. With this model we explain the entire microwave spectrum of all but 1 of the events.

Subject headings: Plasmas - Sun: Bursts - Sun: Microwaves - Sun: X-Rays 


\section{INTRODUCTION}

The association of impulsive microwave and hard X-ray bursts from solar flares has been known since the first observation of an impulsive hard X-ray burst (Peterson and Winkler 1959). Close time correlation of these emissions was established (Kundu 1961) and later improvements in the time resolution of the observations (revealed common time structures on a scale as short as $1 \mathrm{~s}$ (Frost 1969; Kane and Anderson 1970; McKenzie 1972; Kane 1972; Cranne11 et al. 1978; Wiehl and Matzler 1980). The microwave emission is believed to be gyrosynchrotron radiation from energetic electrons gyrating along the magnetic . field lines that thread solar plasmas; the hard $X$ rays are electron-ion collisional bremsstrahlung produced by electrons of comparable energy. The close time correlation and similar electron energy ranges suggest that both emissions come from the same population of electrons. Because the X-ray yield and microwave emissivity are sensitive to physical conditions in the flare plasma, comparison of associated hard X-ray and microwave emissions, event-by-event, provides a critical test of competing flare models (cf. Takakura and Kai 1966; Holt and Ramaty 1969; Takakura 1972; Matzler et al. 1978; Kane et al. 1980; Kundu and Vlahoṣ 1982).

Two crucial issues remain to be settled concerning the physics of these emissions. The first is whether both are produced by the same distribution of electrons. The second is whether thermal or nonthermal electrons produce the emissions.

Regarding the first question, only analysis of coincident hard X-ray and microwave images can give a conclusive answer. Only one flare with 2 dimensional mapping in both microwaves and hard $X$ rays has been described in the literature (Hoyng et al. 1983). They concluded that a single power-law dist- 
ribution of electrons could have produced both emissions, with the $\mathrm{X}$ rays originating near the footpoints and the microwaves coming from the top of a single loop. Statistical uncertainties in the data were large, however, and more complex models could not be excluded. While some other flare X-ray images place the sources at the footpoints of loops (Hoyng et al. 1981a, b; Duijveman, Hoyng and Machado 1982), there are observations of impulsive X-ray sources at. the tops of flare loops or filling one leg (Ohki et al. 1982; Tsuneta et al. 1982; Takakura et al. 1982; Kawabata et al. 1982). In some cases, 1-dimensional interferometric microwave observations were also made, suggesting that the microwaves and $\mathrm{X}$-rays usually come from the same location (Takakura et al. 1982; Kawabata et al. 1982). Because the question of source locations remains unsettled, and particularly because time resolution and spectral coverage are sacrificed in present imaging observations, it is worthwhile to exploit to the fullest the available data obtained without spatial resolution. Observations with fine time resolution and broad spectral coverage but without spatial resolution have recently been made, and some important inferences about the structure of flares can be drawn from their analysis. Spectral analysis of these observations also allows deductions of the source electron distributions to be made, which may settle the second question.

In this paper we present the results of a study of 13 flares observed with the Berne Radio Observatory and with the Hard X-Ray Burst Spectrometer (HXRBS) on the Solar Maximum Mission spacecraft (SMM). The dynamic spectra of both emissions were compared, on an event-by-event basis, and properties of the spectra at the time of maximum microwave flux were compared from event to event. The results were analyzed in an effort to address the issues stated above. Concerning the first issue, we find new evidence for a close connect- 
ion between the source electrons for the hard $X$ rays and the microwaves. Interpretation of the microwave spectra is a complex process, however, because gradients in the magnetic field strength and in the electron distribution within the source both contribute to the observed microwave spectral properties (Schochlin and Magun 1979). This complexity may explain the results of Marsh et al. (1981), who concluded that no single-temperature or single power-1aw electron spectrum could be the sole source of both the hard X-ray and microwave spectra of a flare observed with HXRBS and the Very Large Array (VLA). Our results also contain evidence for important thermal contributions to impulsive microwave and hard X-ray emissions. A thermal origin is not widely attributed to impulsive microwave bursts. However, the hypothesis that a common, single-temperature electron distribution dominated the hard $\mathrm{X}$-ray and microwave emissions allowed us to deduce an $\mathrm{X}$-ray source area of one of the flares in agreement with the area measured by the Solar hard X-ray Telescope (SXT) aboard the Hinotori spacecraft (Ohki et al. 1982). This is the only flare for which such a comparison could be made.

The selection criteria for the present study were designed to take optimum advantage of the broad spectral coverage at microwave frequencies available for the first time for a large sample of solar flares. The events analyzed in this work were selected because of their large microwave peak fluxes. Their peak fluxes were $>500 \mathrm{SFU}$, thus qualifying them as Great Microwave Bursts (GMBs) according to the standard criteria of solar radio astronomy (Solar Geophysical Data 1982). Attention was restricted to events which exhibited a relatively simple microwave time structure at low frequency, and to events observed with the HXRBS.

of the many flare models in the literature, we chose to test the single- 
temperature interpretation of Crannell et al. (1978) and a multi-thermal model developed by Dulk and Dennis (1982). Our interest in thermal models is spurred by several recent observations and theoretical developments. First, one of the primary predictions of the popular nonthermal beam model (Brown 1971, 1975) is a strong brightening of the observed hard $X$ rays with energy greater than $300 \mathrm{keV}$ from flares near the solar 1imb; this limb brightening 'has been searched for in observations with the Gamma Ray Spectrometer on SMM and found to be absent (Zolcinski et al. 1982). This result is consistent with a source electron distribution which is isotropic, such as in thermal models. Anisotropic emission has also been sought in single flares by comparing observations made from widely separated points in space (Kane 1980; WiehI and Desai 1983), with negative results to date.

Secondly, the results of theoretical and experimental studies of proposed impulsive-phase flare energy release mechanisms sugges $\tau$ that heating of the flare plasma to temperatures of order $10^{8} \mathrm{~K}$ occurs, rather than acceleration of large numbers of particles. It is generally agreed that release of energy stored in magnetic fields is the only process capable of supplying the energy released in flares. Known mechanisms of release of the stored energy via magnetic field reconnection are only capable of depositing $10 \%$ or less of the energy released into kinetic energy of accelerated particle beams, with the remaining $90 \%$ or more appearing as heat (cf. Smith 1980; Spicer and Brown 1981). The thermalization of electrons is much faster than would be expected from Coulomb self-collision time considerations because the heating is due predominantly to plasma turbulence. Therefore, strong thermal contributions to the emissions are to be expected. Thermal models have been developed by Brown (1974); Matzler et al. (1978); Matzler (1978); Smith and Lilliequist 
(1979); Brown, Melrose and Spicer (1979); Brown, Craig and Karpen (1980) and others.

It should be noted that the common observation of power-law X-ray spectra is not adequate to establish the nonthermal distribution of flare electrons. Multi-thermal electron populations can give rise to power-law X-ray spectra (Chubb et al. 1966; Brown 1974; Colgate 1978). Exponential spectra which can be. fitted by a single-temperature thermal bremsstrahlung function are also common (Crannell et al. 1978; Elcan 1978). Such exponential spectra may originate in a single-temperature plasma, but nonthermal processes are capable of producing them as well (cf. Kaplan et al. 1974). We have tested the $X$-ray spectra of the events of the present study for acceptable fits to both the thermal and power-law forms, but the significance of the spectral parameters so derived is model-dependent.

The multi-thermal model of Dulk and Dennis (1982) attempts to explain both the power-law X-ray spectrum and the microwave spectrum at frequencies below the peak frequency by means of a plausible distribution of temperatures and magnetic fields. We will show that its predictions are inconsistent with the properties of most of the events in this study. This may be because the particular geometry chosen for the source region is not appropriate. We show how the geometry can be changed to correspond with that of the leg of a flaring loop. Some VLA observations are suggestive of such a source region (e.g. Kundu, Schmahl and Velusamy 1982). The modified geometry can account for all but one of the microwave spectra.

In'§2, details of the event selection, microwave and hard X-ray instrumentation and calibration, and hard $\mathrm{X}$-ray spectral deconvolution are set forth. Techniques of temporal and spectral analysis which were applied to 
the microwave data are described in $\$ 3$. The hard $\mathrm{X}$-ray analysis is described in $\$ 4$. In $\$ 5$, the derivation of source parameters is presented based on the assumption that both the $\mathrm{X}$-ray and microwave emissions originate in a common thermal source with temperatures of the order of $30 \mathrm{keV}$. The predictions of the hemispherical thermal model of Dulk and Dennis involving plausible nonuniformities in the temperature and magnetic field within the source are also compared with the observations, possible absorption processes are discussed, and a new nonuniform model employing diverging magnetic fields is developed. In $\$ 6$, the temporal behaviors as functions of microwave frequency and $\mathrm{X}$-ray photon energy of the-respective emissions are parameterized for each event and compared from event to event. Spectral parameters and other derived parameters are also correlated. The correlations found in $\$ 6$ are interpreted in $\$ 7$, yielding numerous mode1-independent and mode1-dependent results. In $\S 8$, overall conclusions of the study are summarized. 


\section{DATA}

\subsection{Event Selection}

Solar cycle 21, which began in 1976 June, peaked in late 1979 at a smoothed sunspot number of 165 . This is well above the mean peak number of 105 for cycles 8 through 20 (Coffey 1982). From the many hundreds of microwave bursts recorded at Berne during the period of peak sunspot number, we selected all events having a flux in excess of 500 SFU (I SFU $=10^{-22} \mathrm{~W}$ $\mathrm{m}^{-2} \mathrm{~Hz}^{-1}$ ) in at least one of the frequency bands in our operating range of 3.2 to $92.5 \mathrm{GHz}$. This flux threshold corresponds to the definition of a GMB (SGD 1982). During the interval from 1980 January until 1981 December, the Berne Radio Observatory recorded observations of 61 GMB in digita1 form. We found that 26 of these 61 GMBs were also observed with the HXRBS on SMM.

These 26 events were examined to determine whether or not their microwave time histories were dominated by a single rise and fall in emission during each event, and each event was labelled "simple" or "complex", accordingly. In the present work we chose to concentrate on the "simple" flares in the hope that they would be easier to understand. Of the above 26 events, 13 had time histories which were dominated in the frequency range from 3 to $8 \mathrm{GHz}$ by a single peak with possible superposed fluctuations of $<10 \%$ of the peak flux. In some of these events, there was evidence for complexity at high frequencies, but even at those frequencies a single peak dominated the emission at the time of maximum flux. These 13 events were labeled "simple", and time histories of them at representative frequencies are shown in Figures 1 through 13, together with time histories of the hard X-ray flux and effective electron temperature computed from the $X$-ray spectra (see $\$ 4.2$ ). 
TABLE 1: GMBS WITH SIMPLE TIME PROFILES

\begin{tabular}{|c|c|c|c|c|c|c|c|}
\hline $\begin{array}{r}\text { EVENT } \\
\text { NUMBER }\end{array}$ & \multirow[t]{2}{*}{ DATE } & \multirow{2}{*}{$\begin{array}{l}\text { TIME OF MAX. } \\
\text { EMISSION } \\
\text { (UT) }\end{array}$} & \multirow[t]{2}{*}{$\begin{array}{c}\text { H } \alpha \text { FLARE (a) } \\
\text { POSITION }\end{array}$} & \multirow{2}{*}{$\begin{array}{l}\text { RADIO (b) } \\
\text { SPECTRAL } \\
\text { TYPE }\end{array}$} & \multicolumn{2}{|c|}{$\begin{array}{c}\text { PARTICLES (c) } \\
\text { EVENTS }\end{array}$} & \multirow{2}{*}{$\begin{array}{c}\text { GRS (d) } \\
\text { EVENTS } \\
>0.3 \mathrm{MeV} \\
\end{array}$} \\
\hline & & & & & e- & p+ & \\
\hline 1 & 1980 Mar 27 & $13: 00$ & $\mathrm{~N} 29 \mathrm{E} 70\left(^{\mathrm{e}}\right)$ & NONE & $\mathrm{N}$ & $\mathrm{N}$ & $\mathrm{N}$ \\
\hline 2 & 1980 Mar 29 & $09: 18$ & N27E38 & IIIGG & $\mathrm{N}$ & $\mathrm{N}$ & $\mathrm{Y}$ \\
\hline 3 & 1980 Mar 29 & $09: 55$ & $\mathrm{~N} 12 W 13$ & IIIGG & $\mathrm{N}$ & $N$ & $\mathrm{Y}$ \\
\hline 4 & 1980 Jun 4 & $06: 55$ & S13E59 & NONE & $\mathrm{N}$ & $P$ & $\mathrm{Y}$ \\
\hline 5 & 1980 Jun 29 & $10: 42$ & s27w90 & IIIGG & $\mathrm{P}$ & $\mathrm{P}$ & $\mathrm{Y}$ \\
\hline 6 & $1980 \mathrm{JuI} 1$ & $16: 27$ & S12W38 & II & $\mathrm{N}$ & $\mathrm{N}$ & $\mathrm{Y}$ \\
\hline 7 & 1980 Nov 6 & $06: 51$ & N09E08 & NONE & $\mathrm{N}$ & $P$ & $\mathrm{~N}$ \\
\hline 8 & 1981 Apr 15 & $06: 44$ & N20W65 & IIIG & $\mathrm{N}$ & $\mathrm{N}$ & $\mathrm{Y}$ \\
\hline 9 & 1981 Apr 18 & $10: 51$ & $\begin{array}{l}\text { NO FLARE } \\
\text { OBSERVED }\end{array}$ & IIIG & $\mathrm{N}$ & $N$ & $\mathrm{Y}$ \\
\hline 10 & 1981 Apr 26 & $11: 16$ & N12W74 & IIIG & - & $P$ & N \\
\hline 11 & 1981 Apr 26 & $11: 54$ & N16W75 & $I I I G, I V$ & - & $\mathrm{P}$ & $Y$ \\
\hline 12 & 1981 Aug 10 & $06: 59$ & S13W15 & IIIG & $\mathrm{N}$ & $\mathrm{N}$ & $\mathrm{Y}$ \\
\hline 13 & 1981 Dec 7 & $14: 51$ & S06E90(f) & NONE & $\mathrm{N}$ & $\mathbf{N}$ & $\mathrm{Y}$ \\
\hline
\end{tabular}

(a) Flare positions: SGD reports; Helen E. Coffey, NOAA, private communication.

(b) Radio spectral type: SGD prompt Reports, NOAA GG indicates Large group of $>10$ bursts, G indicates small group of $<10$ bursts.

(c) Particle events: Solar Maximum Year Flare List, Rust et al. (1982) $\mathrm{N}$ indicates No, $\mathrm{P}$ indicates Possible, $\mathrm{Y}$ indicates Yes, and "-" implies no observations available.

(d) SMM/Gamma Ray Spectrometer (GRS) Data, courtesy D.J. Forrest

(e) indicates three events in progress at the same time

(f) SGD Preliminary Report, 1981 December 15 
The analysis and interpretation of these 13 "simple" bursts are the subject of the present work. The associated $H \alpha$, radio, particle and $\gamma$-ray observations are summarized in Table 1. The 13 GMBs are, in most cases, associated with Type III radio bursts and with $\gamma$ rays above $0.3 \mathrm{MeV}$, but were not associated with large particle events. Of the 13 events studied, 4 events had associated $H \alpha$ flares in the eastern hemisphere of the Sun, making it unlikely that prompt particle events could be observed. Seven events had flare sites in the western hemisphere, making it likely that the Earth was well connected magnetically, and, therefore, probable that any prompt particle events could have been observed. of those 7 events, 4 flares were definitely not associated with particle events. For the other 3 flares, particle events could not be ruled out entirely, but any that were produced must have been relatively weak. Consequently, we believe that these bursts were produced in confined sources in which most of the particles remain trapped.

On the basis of the microwave time profiles and the adopted selection criteria we regarded events No, 10 and 11 as two separate bursts. The X-ray observations, however, indicate flare emission starting with event No. 10 and continuing through event No. 11, without returning to the background level. On this basis, therefore, event No. 10 might be considered as the impulsive phase and event No. 11 as a second stage of a single event.

\subsection{Microwave Instrumentation and Calibration}

The facilities and instrumentation of the Berne Radio Observatory are described by Magun et al. (1981). The following is a summary of those facts pertinent to an understanding of the analysis presented in this paper.

The temporal resolution changes from $1 \mathrm{~s}$ before a flare to $0.1 \mathrm{~s}$ during 
the flare. Absolute timing with an accuracy of $100 \mu \mathrm{s}$ is derived from a standard frequency reference transmitted from Prangins, Switzerland.

During the observations of the first three simple events, microwave intensities were measured at $8.4,10.4,11.8,19.6$ and $35.0 \mathrm{GHz}$. After the 3rd event, the $10.4 \mathrm{GHz}$ radiometer was replaced by the $5.2 \mathrm{GHz}$ polarimeter. In addition to the intensities measured at these different frequencies, circular polarization was also recorded with all receivers except those at $10.4 \mathrm{GHz}$ and $11.8 \mathrm{GHz}$. Starting in early 1981, an additional polarimeter at $3.2 \mathrm{GHz}$ was put into operation. In order to supplement our observations at lower microwave frequencies, for events prior to the implementation of our 3.2 and $5.2 \mathrm{GHz}$ polarimeters, we used the time profiles at $2.8 \mathrm{GHz}$ published in the ottawa monthly reports.

The 92.5-GHz polarimeter was in operation during the entire interval, but did not indicate any flux above quiet-Sun levels for any of the simple events. No spatial information was obtained for the present work since the beam width at all frequencies except $92.5 \mathrm{GHz}$ was greater than the diameter of the solar disk.

The absolute fluxes were determined in the following manner: the microwave flux. was recorded as a percentage of the quiet Sun flux at each frequency with three automatic calibrations daily. For the days of burst activity, absolute quiet-Sun fluxes were obtained at $2.8 \mathrm{GHz}$ from Ottawa; at $1.0,2.0$, 3.75 and $9.4 \mathrm{GHz}$ from Toyokawa; at 1.47 and $9.5 \mathrm{GHz}$ from Berlin; and at 17 GHz from Nobeyama. These values were then used to construct a spectrum of the quiet-Sun flux in the range from 1 to $17 \mathrm{GHz}$ for each day of interest. -The interpolated quiet Sun values at our frequencies were used to determine the absolute flux levels from the original relative Berne values. The quiet- 
Sun flux at $35.0 \mathrm{GHz}$ was assumed to be constant at $2400 \mathrm{SFU}$.

\subsection{Hard X-Ray Instrumentation and Spectral Analysis}

The hard X-ray data were obtained with the HXRBS on the SMM spacecraft. This instrument is described in detail by Orwig, Frost, and Dennis (1980). Count rates were recorded for fifteen channels distributed over the instrument's energy range, which has varied slowly and monotonically since launch. In 1980 March the energy range was 26 to $456 \mathrm{keV}$, and in 1981 December it was 30 to $531 \mathrm{keV}$. A description of this behavior and a listing of events observed with HXRBS are available in Dennis et al. (1983).

$\mathrm{X}$-ray data used in this study were collected with a time resolution of $0.128 \mathrm{~s}$. We used count rates averaged over $1 \mathrm{~s}$ intervals or longer in the spectral analysis of this study in order to reduce uncertainties in the fitting parameters due to photon counting statistics to less than - $10 \%$.

Representations of the incident hard X-ray spectra were obtained from the measured count rates by the use of a software model of the HXRBS response function. Physical properties of the detector and characteristics of the pulse-height analyzer were employed to compute the instrument response to various assumed incident spectra. The response of the instrument is complex because of the numerous types of interactions which are possible in the CsI(Na) crystal, the aluminum window, and the dead layer on the crystal. The most probable type of photon interaction is photoelectric over most of the applicable energy range; but $\mathrm{K}$-escape, Compton scattering, and rejection of counts by the anticoincidence shield can change the detector's sensitivity significantly. The energy resolution of the detector causes spreading of counts into a given channel from its neighbors and vice versa. Another complicating factor at high count rates is pulse pile-up, which occurs when 
several counts arrive within the shurtest time $(2 \mu s)$ resolvable with the pulse-height analyzer. The pulse pile-up effects are artifacts of the pulse counting system of the instrument and were modeled using the statistical techniques of Datlowe $(1975,1977)$. The spectra we present are corrected for alI of these effects.

The following two types of incident spectra were assumed: the power-law form

$$
I(E)=K_{\gamma}(E / 50)^{-\gamma}
$$

and the thermal bremsstrahlung function

$$
I(E)=K_{T} E^{-1} T^{-0.5} G_{E} \exp [-(E-50) / T],
$$

where $K_{\gamma}, \gamma, K_{T}$ and $T$ are fitting parameters, $E$ is the photon energy in $\mathrm{keV}, \mathrm{T}$ is the temperature in $\mathrm{keV}$, and $\mathrm{I}(\mathrm{E})$ is the $\mathrm{X}$-ray flux in photons $\mathrm{cm}^{-2}$ $\mathrm{s}^{-1} \mathrm{keV}^{-1}$.

The thermal emission measure, EM, can be calculated from $\mathrm{T}$ and $\mathrm{K}_{\mathrm{T}}$ as follows:

$$
\text { . } \mathrm{EM}=9.310^{41} \mathrm{~K}_{\mathrm{T}} \exp (50 / \mathrm{T}) \mathrm{cm}^{-3} .
$$

The total effective Gaunt factor, $G_{E}\left(=G \overline{Z^{2}}\right)$, was determined for temperatures less than $5 \times 10^{8} \mathrm{~K}$ from Groenschild and Mewe (1978) and Mewe (private communication) assuming solar abundances giving the average value of $\mathrm{Z}^{2}=1.355$. For temperalures greater than $5 \times 10^{8} \mathrm{~K}$, the Gaunt factor was calculated using the expressions given by Matteson (1971). For the power-law and the thermal forms, tables of convolved spectra were constructed for various $K, \gamma$ and $T$ values using the software model mentioned above. The values were chosen so that interpolation could be used to obtain spectra for intermediate $\grave{\mathrm{K}}_{\gamma}, \gamma$ or $\mathrm{K}_{\mathrm{T}}, \mathrm{T}$ pairs, with $\approx 10 \%$ accuracy. To find a least-squares representation of the incident spectrum, the following iterative method was 
used: initial values of $\left(\mathrm{K}_{\gamma}, \gamma\right)$ or $\left(\mathrm{K}_{\mathrm{T}}, \mathrm{T}\right)$ were chosen and the corresponding convolved spectrum was computed from the table. For each of the 15 channels, a conversion factor was found to convert observed counts $s^{-1}$ into incident photons $\mathrm{cm}^{-2} \mathrm{~s}^{-1} \mathrm{keV}^{-1}$. Each observed count rate (minus the pre-flare background rate) was divided by this ratio to estimate the true incident flux. Finally, the result was fitted to a power-law or thermal . function to obtain new $\left(K_{\gamma}, \gamma\right)$ or $\left(K_{T}, T\right)$ pairs such that $\chi^{2}$ was minimized. These new parameters were then used to generate the convolved spectrum in the next iteration. This procedure was found to converge rapidly, usually in two or three iterations.

It should be noted that we have excluded data in Channel 1 from all spectral fits on the grounds that its calibration is poorly known. The resulting fitted parameters are believed to be accurate to within 10\%. Deconvolved HXRBS flare spectra have been compared with spectra obtained from simultaneous observations with an X-ray detector on ISEE-3 resulting in agreement to better than $20 \%$ (Kane, private communication). 


\section{MICROWAVE OBSERVATIONS}

In this section we present values of parameters that characterize the microwave temporal behavior and the microwave spectra at the time of maximum microwave flux. These parameters are used in the correlation. analysis of $\$ 6$.

\subsection{Temporal Structures}

Three time histories of each event at different microwave frequencies are shown in Figures 1 through 13, together with the corresponding hard X-ray time histories. Three times were computed to characterize each time history: the rise time, fall time and characteristic time. For both microwave and hard X-ray events, the rise and fall times are the times taken for the flụx to change from $25 \%$ to $100 \%$ of the peak flux. In Tables 2 and 3 the microwave rise $\left(\tau_{\mu r}\right)$ and fall times $\left(\tau_{\mu f}\right)$ measured in this way are given for all 13 events.

Because of the spikiness of some events, particularly at high frequencies, the above definition of rise and fall times can sometimes give misleading results. To avoid this problem we also computed a characteristic time, $\tau_{\mu c}$, for each event defined by the following expression:

$$
\tau_{\mu C}=S_{\max }^{-1} \sum S(t) \Delta t
$$

where $S_{\max }$ is the maximum flux, $S(t)$ is the measured flux as a function of time, $t$, and the sum is taken over all time intervals, $\Delta t$, for which there was significant flux above pre-flare levels. This is approximately equivalent to the full width at half maximum of the event. Table 4 gives $\tau_{\mu \mathrm{c}}$ for each event at different frequencies.

Examination of Tables 2 through 4, shows that the rise and fall times, as well as the characteristic times, generally decrease with increasing fre- 


\section{ORIGINAL PAEE TS OF POOR QUALITY}

TABLE 2: MICROWAVE RISE TIMES, $\tau_{\mu r}$, IN SECONDS

\begin{tabular}{|c|c|c|c|c|c|c|c|c|}
\hline \multirow{2}{*}{$\begin{array}{r}\text { EVENT } \\
\text { NUMBER }\end{array}$} & \multicolumn{8}{|c|}{ FREQUENCY (GHz) } \\
\hline & 2.8 & 3.2 & 5.2 & 8.4 & 10.4 & 11.8 & 19.6 & 35.0 \\
\hline 1 & 459 & - & - & 136 & 136 & 112 & - & - \\
\hline 2 & - & - & - & 8 & 7 & 8 & 5 & 4 \\
\hline 3 & - & - & - & 10 & 10 & 11 & 9 & 10 \\
\hline 4 & - & - & 29 & 36 & - & 35 & 35 & 33 \\
\hline 5 & - & - & 36 & 29 & - & 34 & 24 & - \\
\hline 6 & 43 & - & 42 & 29 & - & 32 & 29 & 29 \\
\hline 7 & - . & - & 50 & 18 & - & 15 & 13 & $\approx 14$ \\
\hline 8 & - & 25 & 26 & 25 & - & 20 & 15 & - \\
\hline 9 & - & 93 & 84 & 70 & - & 60 & 59 & 58 \\
\hline 10 & 77 & 62 & 93 & 92 & - & 98 & 98 & - \\
\hline 11 & 843 & 512 & 470 & - & - & 463 & 482 & 584 \\
\hline 12 & - & 16 & 14 & 14 & - & 12 & 9 & $\approx 10$ \\
\hline 13 & - & - & - & 10 & - & 13 & 10 & 9 \\
\hline
\end{tabular}


TABLE 3: MICROWAVE FALL TIMES, $\tau_{\mu f}$, IN SECONDS

\begin{tabular}{|c|c|c|c|c|c|c|c|c|}
\hline \multirow{2}{*}{$\begin{array}{l}\text { EVENT } \\
\text { NUMBER }\end{array}$} & \multicolumn{8}{|c|}{ FREQUENCY (GHz) } \\
\hline & 2.8 & 3.2 & 5.2 & 8.4 & 10.4 & 11.8 & 19.6 & 35.0 \\
\hline 1 & 951 & - & - & 843 & 898 & 930 & - & - \\
\hline 2 & - & - & - & 13 & 9 & 8 & 6 & 6 \\
\hline 3 & - & - & - & 31 & 28 & 25 & 19 & 23 \\
\hline .4 & - & - & 81 & 56 & - & 28 & 23 & 12 \\
\hline 5 & - & - & 28 & 23 & - & 18 & 22 & - \\
\hline $6^{\circ}$ & 121 & $\rightarrow$ & 130 & 122 & - & 109 & 80 & 35 \\
\hline 7 & - & - & 102 & 30 & - & 28 & 31 & $\approx 33$ \\
\hline 8 & - & 81 & 77 & 55 & - & 19 & 25 & - \\
\hline 9 & - & 128 & 112 & 104 & - & 76 & 74 & 83 \\
\hline 10 & 181 & 176 & 171 & 135 & - & 128 & 81 & - \\
\hline 11 & 991 & 997 & 1021 & - & - & 1085 & 1061 & 908 \\
\hline 12 & - & 158 & 69 & 56 & - & 43 & 31 & $\approx 33$ \\
\hline 13 & - & - & - & 35 & - & 37 & 35 & 31 \\
\hline
\end{tabular}


TABLE 4: MICROWAVE CHARACTERISTIC TIMES, $\tau_{\mu \mathrm{C}}$, IN SECONDS

\begin{tabular}{|c|c|c|c|c|c|c|c|}
\hline \multirow{2}{*}{$\begin{array}{r}\text { EVENT } \\
\text { NUMBER }\end{array}$} & \multicolumn{7}{|c|}{ FREQUENCY ( $\mathrm{GHz})$} \\
\hline & 3.2 & 5.2 & 8.4 & 10.4 & $11 . \overline{8}$ & 19.6 & 35.0 \\
\hline 1 & - & - & 426 & 435 & 524 & $\approx 300$ & - \\
\hline 2 & - & - & .24 & 20 & 16 & 10 & - \\
\hline 3 & - & - & 31 & 29 & 27 & 21 & 21 \\
\hline 4 & - & 77 & 66 & - & 56 & 48 & - \\
\hline 5 & - & 50 & 42 & - & 44 & - & - \\
\hline 6 & - & 102 & 96 &,- & 94 & 67 & 41 \\
\hline 7 & - & 136 & 74 & - & 83 & 40 & - \\
\hline 8 & 85 & 83 & 68 & - & 26 & $\approx 30$ & - \\
\hline 9 & 281 & 270 & 195 & - & 154 & 175 & 238 \\
\hline 10 & 217 & 229 & 211 & - & 203 & 165 & - \\
\hline 11 & 1203 & 1187 & - & - & 1203 & 1177 & 1323 \\
\hline $\mathrm{I} 2$ & 123 & 83 & 66 & - & 51 & $\approx 59$ & - \\
\hline 13 & - & - & 29 & - & 36 & 22 & 12 \\
\hline
\end{tabular}


quency. This is shown graphically in Figure 14. This result is a quantitative statement of something that has long been known only qualitatively (Takakura 1975).

\subsection{Spectral Parameters}

The microwave spectra taken at the peak of each of the 13 simple events are shown in Figures 15 to 27 . They were constructed from the calibrated Berne data and from additional microwave observations given in Solar Geophysical Data Comprehensive Reports (Coffey 1980-1981; Coffey 1981-1982). These spectra are classified as C-type (Guidice and Castelli 1975). Each spectrum has a frequency of maximum emission, $f_{\text {peak, }}$ and can be described

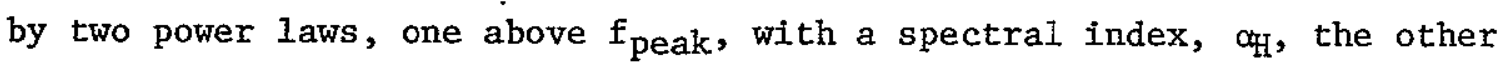
below $f_{\text {peak }}$ with an index, $\alpha_{L}$. The good spectral coverage of our data from 3 to $35 \mathrm{GHz}$ allows us to measure $\alpha_{\mathrm{L}}$ for all 13 events, and $f_{\text {peak }}$ and $\alpha_{\mathrm{H}}$ for all but two of the events. The transition frequency $f_{t}$, below which the flux rises again in the decimeter domain, was estimated from other radio data. It was found to be as $10 \mathrm{w}$ as $0.4 \mathrm{GHz}$ in one event. This compares to the lowest value previously reported of $1.0 \mathrm{GHz}$ (Batchelor, Benz and Wiehl 1983). At the high frequency end of the spectrum we can estimate the frequency, $f_{h}$, at which the flux has decreased to $10 \%$ of the flux at $f_{\text {peak }}$. These spectral parameters are graphically defined in Figure 15. The values of the spectral parameters obtained for each event are listed in Table 5.

\subsection{Polarization}

The Stokes parameters $V$ and $I$ were measured at the following frequencies: $3.2,5.2,8.4,19.6$ and $35.0 \mathrm{GHz}$. ( $\mathrm{V}$ is the excess of left circular polarized flux over right and $I$ is the sum of left and right.) For each event, at the time of maximum flux, we plot in Figure 28 the circular polarization, $\rho_{C}$, 
TABLE 5: MICROWAVE SPECTRAL PARAMETERS

\begin{tabular}{|c|c|c|c|c|c|c|}
\hline $\begin{array}{r}\text { EVENT } \\
\text { NUMBER }\end{array}$ & $\alpha_{L}$ & $\alpha_{H}$ & $\begin{array}{c}f_{t} \\
(G H z)\end{array}$ & $\begin{array}{l}f_{\text {peak }} \\
(\mathrm{GHz})\end{array}$ & $\frac{\mathrm{f}_{\mathrm{h}}}{(\mathrm{GHz})^{\prime}}$ & $\begin{array}{c}S\left(f_{\text {peak }}\right) \\
\text { SFU }\end{array}$ \\
\hline 1 & 1.4 & -4.5 & 1.0 & 8.4 & 18 & 900 \\
\hline 2 & 2.0 & -1.7 & 1.7 & 11.8 & $>50$ & 1100 \\
\hline 3 & 1.4 & $<-0.6$ & 3.5 & 19.6 & $>50$ & 580 \\
\hline 4 & $1.7 / 3.1$ & -0.5 & $\approx 1.0$ & 11.8 & $>50$ & 1200 \\
\hline 5 & 0.9 & -1.4 & 0.8 & 8.4 & 45 & 780 \\
\hline 6 & $0.5 / 1.1$ & - & $\leqslant 0.9$ & $\geqslant 35.0$ & $>50$ & $\geqslant 3100$ \\
\hline 7 & 2.7 & -2.0 & $\approx 2.0$ & 11.8 & 40 & 1400 \\
\hline 8 & 1.7 & -3.7 & 0.8 & 10.7 & 33 & 820 \\
\hline 9 & 2.1 & -0.5 & 1.5 & 11.8 & $>50$ & 690 \\
\hline 10 & 0.4 & $-1 \cdot 0$ & $\leqslant 0.4$ & 2.8 & 36 & 510 \\
\hline 11 & 2.1 & -0.9 & $\approx 0.5$ & 5.2 & $>50$ & 12000 \\
\hline 12 & 0.8 & -1.9 & 0.6 & 11.8 & 50 & 780 \\
\hline 13 & 1.2 & - & $\leqslant 8.4$ & $\geqslant 35.0$ & $>50$ & $\geqslant .770$ \\
\hline
\end{tabular}


as a function of frequency, where $\rho_{c}$ is defined by the relation

$$
\rho_{c}=\left(V-V_{0}\right) /\left(I-I_{0}\right) \text {, }
$$

and $V_{0}$ and $I_{0}$ are the values of the Stokes parameters prior to the burst. At low frequencies the circular polarization is small, as expected for optically-thick sources. At higher frequencies $\rho_{c}$ is sometimes higher, 10 to $20 \%$, as expected for optically-thin sources (Dulk and Marsh 1982). In 5 cases the sense of circular polarization is right handed only, in 4 cases it is left handed only, and in 3 cases it changes sense from one frequency to another during the flare. 


\section{HARD X-RAY OBSERVATIONS}

In this section we present values of parameters that characterize the $\mathrm{X}$-ray temporal behavior and the $\mathrm{X}$-ray spectra at the time of maximum microwave flux. Because the relationship between the $X$-ray and the microwave maxima is of interest in constructing flare models, we also characterize the X-ray spectrum at both the time when it is most intense and at the time when it is hardest. In all cases, the spectrum was hardest near the time of maximum intensity so that each event exhibited a soft-hard-soft spectral evolution.

\subsection{Temporal Parameters}

Two hard X-ray time profiles (Channels 3 and 7) of each event are shown in Figures 1 through 13, together with three microwave time profiles. The $X-r a y$ fluxes used in the time profiles are the estimated photon fluxes obtained from the best-fit power-law spectra. The rise and fall times were measured from these time profiles in the same manner as that used for the microwave parameters, i.e. from a quarter peak to peak value. The hard $X$-ray rise times, $\tau_{x r}$, for channels 1 through 7 are given in Table 6 , and the fall times, $\tau_{x f}$, are given in Table 7. We also computed the hard $\mathrm{x}$-ray characteristic times, $\tau_{x c}$, according to Equation ( 4 ), substituting the measured X-ray flux I for S. These are given in Table 8 and are plotted as a function of energy in Figure 29 for each of the 13 events.

\subsection{Spectral Parameters}

For analytic simplicity, two different functional forms were assumed for the incident X-ray spectra. First the spectrum was assumed to be that expected for thermal bremsstrahlung and second it was assumed to be a single power law (Section 2.3). As noted in $\$ 1$, the results should not necessarily 
ORIGINAL PAQE IS

OF POOR QUALITY

Table 6: HARD X-RAY RISE TIME, $\tau_{x r}$, IN SECONDS

\begin{tabular}{|c|c|c|c|c|c|c|c|}
\hline \multirow[b]{2}{*}{$\begin{array}{r}\text { EVENT } \\
\text { NUMBER } \\
\end{array}$} & \multicolumn{7}{|c|}{ CHANNEL NUMBER } \\
\hline & 1 & 2 & 3 & 4 & 5 & 6 & 7 \\
\hline 1 & 466 & 440 & 405 & 69 & 69 & 103 & 69 \\
\hline 2 & 7 & 7 & 6 & 5 & 5 & - & 4 \\
\hline 3 & 8 & 8 & 7 & 9 & 10 & 8 & 8 \\
\hline 4 & 13 & 15 & 13 & 10 & 11 & 9 & 10 \\
\hline 5 & 15 & 15 & 15 & 11 & 9 & 22 & 35 \\
\hline 6 & 34 & 37 & 33 & 30 & 30 & 30 & 29 \\
\hline 7 & -15 & -15 & -15 & -15 & - & - & - \\
\hline 8 & 2.3 & 17 & 15 & 15 & 13 & 9 & -11 \\
\hline 9 & 112 & 112 & 123 & 53 & 53 & 45 & 45 \\
\hline 10 & 118 & 97 & 46 & 46 & 39 & 39 & - \\
\hline 11 & 247 & 258 & 220 & 220 & 240 & 240 & 240 \\
\hline 12 & 10 & 11 & 10 & 7 & 5 & 5 & 5 \\
\hline 13 & 11 & 8 & 8 & 8 & 8 & 8 & 5 \\
\hline
\end{tabular}


Table 7: HARD $\dot{x}-$ RAY FALL TIME, $\tau_{x f}$, IN SECONDS

\begin{tabular}{|c|c|c|c|c|c|c|c|}
\hline \multirow[b]{2}{*}{$\begin{array}{r}\text { EVENT } \\
\text { NUMBER } \\
\end{array}$} & \multicolumn{7}{|c|}{ CHANNEL NUMBERS } \\
\hline & 1 & 2 & 3 & 4 & 5 & 6 & 7 \\
\hline 1 & -371 & -328 & -345 & -336 & -310 & -284 & -200 \\
\hline 2 & 5 & 5 & 5 & 4 & 4 & - & 4 \\
\hline 3 & 10 & 10 & 10 & 8 & 5 & 5 & 5 \\
\hline 4 & 38 & 34 & 31 & 33 & 32 & 31 & 31 \\
\hline 5 & 51 & 47 & 39 & 37 & 39 & 24 & 11 \\
\hline 6 & 136 & 123 & 65 & 63 & 62 & 62 & 20 \\
\hline 7 & $\sim 24$ & -24 & -24 & -24 & - & - & - \\
\hline 8 & 7 & 7 & 7 & 7 & 6 & 6 & 7 \\
\hline 9 & $>54$ & $>54$ & 33 & 22 & 25 & 27 & 20 \\
\hline 10 & $>72$ & $>72$ & 72 & 72 & 51 & 51 & - \\
\hline 11 & $>360$ & $>345$ & $>345$ & $>330$ & $>300$ & $>300$ & $>300$ \\
\hline 12 & 33 & 28 & 20 & 14 & 13 & 29 & 28 \\
\hline 13 & $\cdot 18$ & 12 & 7 & 6 & 5 & 8 & 4 \\
\hline
\end{tabular}


ORIGINAL PASTS

OF POOR QUALIT'

TABLE 8: HARD X-RAY CHARACTERISTIC TIME, $\tau_{\mathrm{XC}}$, IN SECONDS

\begin{tabular}{|c|c|c|c|c|c|c|c|c|c|}
\hline \multirow[b]{2}{*}{$\begin{array}{r}\text { EVENT } \\
\text { NUMBER } \\
\end{array}$} & \multicolumn{9}{|c|}{ CHANNEL NUMBER } \\
\hline & 1 & 2 & 3 & 4 & 5 & 6 & 7 & 8 & 9 \\
\hline 1 & 505 & 401 & 304 & 262 & 252 & 270 & 172 & 184 & 151 \\
\hline 2 & 14 & 14 & 11 & 11 & 10 & - & 9 & 8 & 8 \\
\hline 3 & 13 & 14 & 12 & 12 & 11 & 11 & $\cdot 11$ & 9 & 10 \\
\hline 4 & 40 & 37 & 28 & 26 & 24 & 22 & 21 & 20 & 20 \\
\hline 5 & 51 & 45 & 37 & 35 & 34 & 33 & 31 & 29 & 25 \\
\hline 6 & 99 & 88 & 56 & 47 & 44 & 43 & 41 & 36 & 35 \\
\hline 7 & 47 & 37 & 26 & 24 & 26 & 24 & 26 & 18 & - \\
\hline 8 & 19 & 18 & 13 & 12 & 11 & 9 & 7 & 11 & 7 \\
\hline 9 & 92 & 86 & 65 & 56 & 48 & 45 & 42 & 38 & 25 \\
\hline 10 & 124 & 107 & 85 & 78 & 86 & 70 & 72 & 80 & 53 \\
\hline 11 & 406 & 397 & 376 & 381 & 368 & 365 & 378 & 362 & 353 \\
\hline 12 & 33 & 29 & 21 & 18 & 18 & 21 & 19 & 16 & 19 \\
\hline 13 & 18 & 18 & 12 & 12 & 10 & 12 & 10 & 12 & - \\
\hline
\end{tabular}


be taken to imply that the electron distributions are thermal or nonthermal, because multi-thermal electron populations can give rise to power-1aw X-ray spectra (Chubb et al. 1966; Brown 1974) and nonthermal processes can give rise to exponential X-ray spectra (cf. Kaplan et al. 1974). Fitted temperatures and power-law indices for the two assumed spectral forms are given for each event in Table 9. Plots of the spectra determined at the times of maximum microwave emission for the two assumed functional forms are shown in Figures 30 through 42 for each of the 13 events. Again, it should be noted that the data in channel 1 has been excluded from the fitting routine.

One can decide whether one or both of the two assumed spectral types give acceptable fits to the observations, or whether neither is acceptable, on the basis of the value of $\chi^{2}$ obtained from the least squares fitting procedure. The relevant paramater is $\mathrm{P}\left(>\chi^{2}\right)$, defined as the probability that random fluctuations in the count rates plus uncertainties in the instrument response function would result in a value of $\chi^{2}$ greater than or equal to the value obtained, if the true incident spectrum is of the assumed functional form and if the estimated uncertainties accurately represent the actuál deviations. Taking into account uncertainties of $\pm 10 \%$ in the conversion factors from count rate to photon flux, we adopted the criterion for an acceptable fit of $\mathrm{P}\left(>\chi^{2}\right)>0.1$. With this criterion, it can be seen from Table 9 that, at the time of maximum microwave emission, $t_{\text {pmax }}$, there are 3 events that give acceptable fits to a thermal bremsstrahlung function (Equation (2)) but not to a power-law function (Equation (1)), and there are 4 that give acceptable fits to a power law but not to a thermal function. There are 3 cases in which both functions are acceptable representations of the spectra, and 3 in which neither function is. 
T̃ABLẺ 9: TEMPÉRATUTUES AND POWER LAW_SPECTRAL INDICES

\begin{tabular}{|c|c|c|c|c|c|c|c|c|c|c|c|c|c|c|}
\hline & EVENT NUMBER & 1 & 2 & 3 & 4 & 5 & 6 & 7 & 8 & 9 & 10 & 11 & 12 & 13 \\
\hline $\begin{array}{l}\text { T1me of } \\
\text { Maximum } \\
\text { X-ray } \\
\text { Intens1ty } \\
t_{\text {xmax }}\end{array}$ & $\begin{array}{l}T(k e V) \\
P\left(>x^{2}\right) \\
Y \\
P\left(>x^{2}\right)\end{array}$ & $\begin{array}{c}31 \\
0.08 \\
4.0 \\
0.00\end{array}$ & $\begin{array}{l}44 \\
\frac{0.73}{3.5} \\
0.00\end{array}$ & $\begin{array}{l}61 \\
\frac{0.10}{3.1} \\
0.00\end{array}$ & $\begin{array}{l}39 \\
0.00 \\
3.8 \\
0.04\end{array}$ & $\begin{array}{l}44 \\
0.02 \\
3.5 \\
0.0\end{array}$ & $\begin{array}{l}61 \\
0.00 \\
3.2 \\
0.04\end{array}$ & $\begin{array}{l}24 \\
0.00 \\
4.5 \\
0.42 \\
\end{array}$ & $\begin{array}{l}40 \\
\frac{0.31}{3.7} \\
0.00\end{array}$ & $\begin{array}{l}29 \\
0.00 \\
4.3 \\
0.33 \\
\end{array}$ & $\begin{array}{l}20 \\
0.12 \\
4.8 \\
0.47 \\
\end{array}$ & $\begin{array}{l}54 \\
0.00 \\
3.6 \\
0.00\end{array}$ & $\begin{array}{l}27 \\
\frac{0.39}{4.3} \\
0.17 \\
\end{array}$ & $\begin{array}{ll} & 50 \\
9 & 0.49 \\
3.3 \\
\underline{7} & 0.04\end{array}$ \\
\hline $\begin{array}{l}\text { Time of } \\
\text { Hárdest } \\
\text { X-Ray } \\
\text { Spectrum } \\
t_{x h}\end{array}$ & $\begin{array}{c}T(k e V) \\
P\left(>x^{2}\right) \\
Y_{m \perp n} \\
P\left(>x^{2}\right)\end{array}$ & $\begin{array}{c}46 \\
0.02 \\
3.3 \\
0.81 \\
\end{array}$ & $\begin{array}{l}45 \\
\frac{0.19}{3.5} \\
0.00\end{array}$ & $\begin{array}{l}68 \\
0.99 \\
3.0 \\
0.00\end{array}$ & $\begin{array}{l}48 \\
0.00 \\
3.5 \\
0.39 \\
\end{array}$ & $\begin{array}{l}51 \\
0.01 \\
3.4 \\
0.30 \\
\end{array}$ & $\begin{array}{l}61 \\
0.00 \\
3.2 . \\
0.04\end{array}$ & $\begin{array}{l}26 \\
0.00 \\
.4 .5 \\
0.03\end{array}$ & $\begin{array}{l}40 \\
\frac{0.31}{3.7} \\
0.00\end{array}$ & $\begin{array}{l}38 \\
0.00 \\
3.9 \\
0.00\end{array}$ & $\begin{array}{l}21 \\
0.02 \\
4.7 \\
0.37 \\
\end{array}$ & $\begin{array}{c}75 \\
0.00 \\
3.1 \\
0.63 \\
\end{array}$ & $\begin{array}{c}27 \\
0.39 \\
4.3 \\
0.17\end{array}$ & $\begin{array}{l}64 \\
0.09 \\
2.9 \\
0.75 \\
\end{array}$ \\
\hline $\begin{array}{l}\text { Time of } \\
\text { Maximum } \\
\text { Microwave } \\
\text { Intensity } \\
\mathbf{t}_{\text {umax }}\end{array}$ & $\begin{array}{l}\mathrm{T}_{x}(\mathrm{keV}) \\
\mathrm{P}\left(>\mathrm{x}^{2}\right) \\
\gamma \\
\mathrm{P}\left(>x^{2}\right)\end{array}$ & $\begin{array}{l}.40 \\
0.14 \\
3.5 \\
0.10 \\
\end{array}$ & $\begin{array}{l}45 \\
\frac{0.19}{3.5} \\
0.00\end{array}$ & $\begin{array}{l}61 \\
0.10 \\
3.1 \\
0.00\end{array}$ & $\begin{array}{l}46 \\
0.00 \\
3.5 \\
0.43 \\
\end{array}$ & $\begin{array}{l}44 \\
0.02 \\
3.5\end{array}$ & $\begin{array}{l}61 \\
0.00 \\
3.2\end{array}$ & $\begin{array}{l}24 \\
0.00 \\
4.5 \\
0.42 \\
\end{array}$ & $\begin{array}{l}40 \\
\frac{0.31}{3.9}\end{array}$ & $\begin{array}{ll} & 38 \\
1 & 0.00 \\
& 3.9 \\
2 & 0.00\end{array}$ & $\begin{array}{l}21 \\
0.02 \\
4.7 \\
0.0 .37 \\
\end{array}$ & $\begin{array}{l}74 \\
0.00 \\
3.1 \\
0.82 \\
\end{array}$ & $\begin{array}{l}27 \\
\frac{0.11}{4.3} \\
0.17\end{array}$ & $\begin{array}{l}56 \\
-0.78 \\
3.1 \\
0.26 \\
\end{array}$ \\
\hline $\begin{array}{r}t_{x \max }- \\
\text { Unce }\end{array}$ & $\begin{array}{c}t_{\text {paax }}(s) \\
\text { rtainty (s) }\end{array}$ & $\begin{array}{l}-19.5 \\
\pm 15 \pm\end{array}$ & $\begin{array}{l}-0.5 \\
\pm 0.5 \pm\end{array}$ & $\begin{array}{c}0 \\
+0.5 \pm\end{array}$ & $\begin{array}{r}-6 \\
\pm 1\end{array}$ & $\begin{array}{l}0.6 \\
\pm 1^{\prime}\end{array}$ & $\begin{array}{r}0 \\
\pm 1\end{array}$ & $\begin{array}{r}-5 \\
\pm 5\end{array}$ & $\begin{array}{l}-1.3 \\
\pm 1 \pm\end{array}$ & $\begin{array}{r}-4 \\
\pm 2.5=\end{array}$ & $\begin{array}{r}-44 \\
\pm 6.5\end{array}$ & $\begin{array}{l}-330 \\
\pm 10\end{array}$ & $\begin{array}{l}-3 \\
\pm 1\end{array}$ & $\begin{array}{r}-1.5 \\
\pm 0.5\end{array}$ \\
\hline $\begin{array}{l}c_{x h}-t \\
\text { Unces }\end{array}$ & $\begin{array}{l}E_{\mu \max }(\mathrm{s}) \\
\text { rtainty (s) }\end{array}$ & $\begin{array}{r}44 \\
\pm \quad 15 \pm\end{array}$ & $\begin{array}{l}0.3 \\
\pm 0.5 \pm\end{array}$ & $\begin{array}{l}0.7 \\
+0.5\end{array}$ & $\begin{array}{l}-1.5 \\
\pm 1\end{array}$ & $\begin{array}{r}2.6 \\
\pm 1\end{array}$ & $\begin{array}{c}0 \\
\pm 1\end{array}$ & $\begin{array}{l}5 \\
\pm 5\end{array}$ & $\begin{array}{r}-1.3 \\
\pm 1\end{array}$ & $\begin{array}{c}1 \\
\pm 2.5\end{array}$ & $\begin{array}{l}-3.8 \\
\pm 6.5\end{array}$ & $\begin{array}{l}-54 \\
\pm 10\end{array}$ & $\begin{array}{l}-0.5 \\
\pm 1 \pm\end{array}$ & $\begin{array}{r}2.5 \\
\pm 0.5\end{array}$ \\
\hline $\begin{array}{l}t_{x h}-t \\
\text { Uncer }\end{array}$ & $\begin{array}{l}t_{x \max } \\
r \text { tainty }(s)\end{array}$ & $\begin{array}{r}64 \\
\pm 15 \pm\end{array}$ & $\begin{array}{l}0.8 \\
+0.5 \pm\end{array}$ & $\begin{array}{l}0.7 \\
\pm 0.5\end{array}$ & $\begin{array}{r}4.5 \\
\pm 1\end{array}$ & $\begin{array}{r}2.0 \\
\pm 1\end{array}$ & $\begin{array}{c}0 \\
\pm 1\end{array}$ & $\begin{array}{l}10 \\
\pm 5\end{array}$ & $\begin{array}{c}0 \\
\pm 1\end{array}$ & $\begin{aligned} & 5 \\
\pm & 2.5\end{aligned}$ & $\begin{array}{l}40 \\
\pm 6.5\end{array}$ & $\begin{array}{r}276 \\
\pm 10\end{array}$ & $\begin{array}{l}2.5 \\
\pm 1\end{array}$ & $\begin{array}{r}4.0 \\
\pm 0.5\end{array}$ \\
\hline
\end{tabular}

N.B. Underscored values of $P\left(>x^{2}\right)$ correspond to acceptable fits. 
Table 9 also shows that there is a significant delay in most events between the time of peak $x$-ray flux, $t_{x m a x}$, and the time of peak microwave flux, $t_{\mu \max }$. A time difference is also common between $t_{\mathrm{xmax}}$ and $t_{\mathrm{xh}}$, the time of hardest $x$-ray spectrum. There is a tendency for $t_{x h}$ and $t_{\text {max }}$ to be closer than $t_{x \max }$ and $t_{\mu \max }$, but such is not always the case; the significance of this is discussed in $\$ 7$.

As is shown in Table 9, during the course of a given event the X-ray spectrum is sometimes better represented by a thermal function, sometimes by a power law, sometimes represented acceptably by both forms, and sometimes by neither. The thermal function tends to fit mure spectra at $t_{x m a x}$, and the power law to fit more spectra at $t_{x h}$. For comparison, we note that similar flare observations made with 0 SO-7 indicated that 28 of 38 events were better characterized by a thermal function at $t_{x \max }$ (Elcan 1978). In $\$ 5.1$, we make use of the fit parameters of the $X$-ray spectrum and the microwave' spectrum at $t_{\mu \max }$ in deriving source parameters. 


\section{DERIVED PARAMETERS}

In this section we discuss the parameters that can be derived from the microwave and hard $\mathrm{X}$-ray measurements under the assumption that the source of both types of radiation is plasma in thermal equilibrium. In other words, we assume that the microwaves are gyrosynchrotron radiation and the $\mathrm{X}$ rays are bremsstrahlung from a common thermal source. In $\$ 5.1$ we derive the area of emission at the peak frequency and the corresponding magnetic field for the 6 events in which a single-temperature interpretation of the X-ray spectrum is possible. In $\$ 5.2$ we discuss a hemispherical model developed by Dulk and Dennis (1982) involving nonuniformities in the temperature and magnetic field to explain the low-frequency part of the microwave spectrum. In $\$ 5.3$ we discuss possible absorption processes affecting the low-frequency spectrum. In $\$ 5.4$ we propose a diverging loop model for the microwave source to explain the complete microwave spectrum, both the high- and the low-frequency parts.

For the sake of simplicity, we use the following nomenclature and units in this discussion: $T$ for temperature in units of $\mathrm{keV}$, $f$ for frequency in $\mathrm{GHz}$, $\mathrm{n}$ for density in $10^{8} \mathrm{~cm}^{-3}$, $\mathrm{L}$ or $\mathrm{r}$ for length in $10^{8} \mathrm{~cm}$, A for area in $10^{18}$ $\mathrm{cm}^{2}$, S for microwave flux in solar flux units ( $1 \mathrm{SFU}=10^{-22} \mathrm{~W} \mathrm{~m}^{-2} \mathrm{~Hz}^{-1}$ ), I for hard X-ray flux in photons $\mathrm{cm}^{-2} \mathrm{~s}^{-1} \mathrm{keV}^{-1}, \mathrm{~B}^{-}$for magnetic field strength in gauss, ED for energy density in erg $\mathrm{cm}^{-3}, \mathrm{U}$ for thermal energy in $10^{27}$ ergs, and $\mathrm{EM}$ for emission measure in $10^{45} \mathrm{~cm}^{-3}$.

\subsection{Single--Temperature Source Parameters.}

At $t_{\mu \max }$, the area of the microwave source and the magnetic field strength in the source can be estimated from the microwave and X-ray spectral 
data under the assumption that both emissions originate in a common singletemperature region. 'In order to determine the area, we first consider the microwave spectrum at frequencies $<f_{\text {peak }}$. The microwave source is generally believed to be optically-thick in this range, and the spectrum for a uniform, single-temperature source is given by the relation

$$
S=0.16 \quad f^{2} \text { A T }
$$

(Cranne11 et al. 1978). Thus, the source area at a given time and frequency can be determined from measurements of $\mathrm{S}$ and $\mathrm{T}$. Table 5 shows that the microwave spectrum usually doesn't have this dependence on $f\left(\right.$ i.e. $\alpha_{L} \neq 2$ ). A possible explanation for this is discussed below, but for the present we assume that the flux $S_{\text {peak }}$ at $f_{\text {peak }}$ is dominated by such a single-temperature source. The assumption that the $\mathrm{X}$ rays originate in the same source allows us to substitute the temperature derived from the $x$-ray spectra at $t_{\text {max }}$ ( $T_{X}$ in Table 9) for $T$ in Equation (6). This substitution is valid only when the thermal function is an acceptable representation of the X-ray spectrum at $t_{\text {pmax }}$ i.e. for events $1,2,3,8,12$ and 13 . The area can then be calculated from the following relation derived from Equation (6):

$$
A_{\text {peak }}=6.26 \mathrm{~S}_{\text {peak }} \mathrm{T}_{\mathrm{x}}^{-1} \mathrm{f}_{\text {peak }}^{-2}=10^{-2} \pi \mathrm{r}_{\text {peak }}^{2} \text {. }
$$

Dulk and Marsh (1982) have provided simplified expressions characterizing the microwave emission which are applicable to those events under consideration. The magnetic field strength was determined using the following expression for $f_{\text {peak }}$ obtained from Dulk and Marsh with the numerical constant adjusted for the units used here:

$$
f_{\text {peak }}=4.910^{-3}(\mathrm{n} \mathrm{L})^{0.1}(\sin \theta)^{0.6} \mathrm{~T}_{\mathrm{X}}^{0.7} \mathrm{~B}^{0.9} \text {, }
$$

where $\theta$ is the angle between the magnetic field and the line of sight, and $L$ is the characteristic length of the source. Because of the small power of 
$\mathrm{nL}$, the peak frequency is not very sensitive to the values of $\mathrm{n}$ and $\mathrm{L}$. For typical values of density of $5 \times 10^{8} \mathrm{~cm}^{-3}$ and length of $\approx 10^{9} \mathrm{~cm}$, we obtain (nL) $0.1 \approx 1.5$. We will use this value in what follows, without incurring significant errors. Because the emissivity is proportional to the 6th power of $\sin \theta$, only regions with large $\theta$ contribute significantly to the observed radiation. For example, when $\theta \approx 70^{\circ}$, the magnetic field can $b$ r determined from the following relation:

$$
\mathrm{B}=244 \quad \begin{array}{lll}
\mathrm{f}_{\text {peak }} & 1.11 & -0.78 \\
\mathrm{~T}_{\mathrm{X}}
\end{array}
$$

We will assume $\theta \approx 70^{\circ}$ in what follows.

For those events for which the single-temperature model is applicable, the electron densities can be calculated from the X-ray thermal emission measure and the area of microwave emission, using the following relationship:

$$
\mathrm{n}^{2}=100 \mathrm{EM} \quad \mathrm{A}_{\text {peak }}^{-3 / 2}
$$

(cf. Cranne11 et al 1978). These and other parameters of the source can be determined under the assumption of a common thermal source for microwaves and hard $X$ rays, and these are given in Table 10 . The other derived parameters are as follows:

(a) The energy density at $\mathfrak{r}_{\mu \max }$ calculated from the following expression under the assumption that the ions and the electrons have the same temperature and number density:

$$
\mathrm{ED}=0.48 \mathrm{n} \mathrm{T}
$$

(b) The total thermal energy of the flare plasma at $t_{\text {pmax }}$ calculated from the following expression under the same assumption:

$$
\mathrm{U} \simeq 210^{-3} \mathrm{r}_{\text {peak }}^{3} \mathrm{n} \mathrm{T}
$$

The volume of the source was assumed to be $4 / 3 \pi r_{\text {peak }}^{3}$.

(c) The value of $\beta$, the ratio of gas to magnetic pressure, obtained 
TABLE 10: SINGLE-TEMPERATURE OBSERVED AND DERIVED PARAMETERS

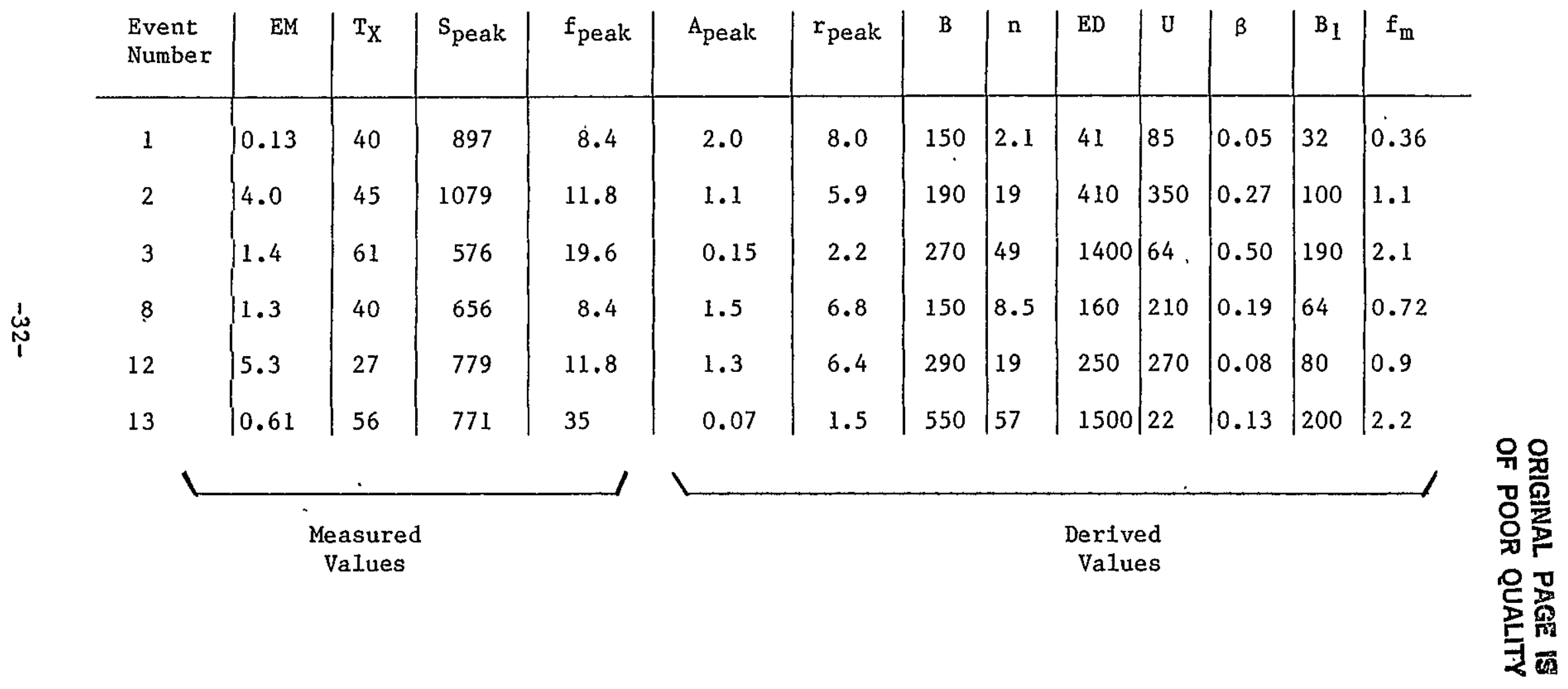


from the previously determined temperature and field strength using the relation

$$
\beta=12 \mathrm{n} T \mathrm{~B}^{-2} \text {. }
$$

The values of $\beta$ are in the range from 0.05 to 0.50 , implying that the magnetic field is capable of confining the plasma.

(d) The minimum magnetic field strength, $B_{1}$, required to confine the source, i.e. for the plasma $\beta$ to be unity:

$$
\mathrm{B}_{1}=3.5(\mathrm{n} \mathrm{T})^{1 / 2} \text {. }
$$

(e) The minimum frequency, $f_{m}$, at which the radiation is expected to be gyrosynchrotron:

$$
f_{\mathrm{m}} \simeq 1.110^{-2} \mathrm{~B}_{1} \text {. }
$$

This expression was derived assuming that the 4 th harmonic of the gyrofrequency is the lower limit of the spectrum. Below the 4th harmonic, gyroresonance absorption is expected to attenuate the emission (Ramaty and Petrosian 1972).

The derived parameters for events with thermal X-ray spectra are of the same order of magnitude as those found for simple impulsive events by Crannell et al. (1978), and are all reasonable for the corona in an active region.

\subsection{A Multi-Temperature Source Model}

From Equation (6) we see that for a single-temperature source of constant area, $S \propto f^{2}$ or $\alpha_{\mathrm{L}}=2$. Examination of Table 5 shows that the observed values of $\alpha_{\mathrm{L}}$ deviate significantly both above and below this predicted value of 2. Of the 13 events studied, 9 have $\alpha_{L}<2,2$ have $\alpha_{L}>2$, and 3 have $\alpha_{L} \simeq 2$. Event number 4 is a special case in that $\alpha_{L}>2$ for $1<f<3 \mathrm{GHz}$ but $\alpha_{\mathrm{L}}<2$ for $3<f<10 \mathrm{GHz}$. The average value of $\alpha_{\mathrm{L}}$ 
for the 13 events is $1.4 \pm 0.4$, a value similar to that found from studies of other events (Schochlin and Magun 1979; Dulk and Dennis 1982, hereinafter DD).

In thermal models, a value of $\alpha_{\mathrm{L}}<2$ can arise only from nonuniformities in the source. A value of $\alpha$ L $>2$ may result from free-free and gyroresonance absorption in regions outside the source, as is discussed in $\S 5.3$.

In this section we describe the model developed by DD to parameterize possible source nonuniformities in the temperature and magnetic field that can give rise to values of $\alpha_{L}<2$. DD developed a model involving a hemisphere in which the temperature and magnetic field decrease with distance, $r$, from the center according to the following power-law relationships:

$$
\mathrm{T}=\mathrm{T}_{\max }\left(\mathrm{r} / \mathrm{r}_{\min }\right)^{-\alpha} \mathrm{T} ; \mathrm{B}=\mathrm{B}_{\max }\left(\mathrm{r} / \mathrm{r}_{\min }\right)^{-\alpha} \mathrm{B} ; \mathrm{r}>\mathrm{r}_{\min },
$$

where $r_{\min }$ is the radius of the hottest core. $T_{\max }$ was assumed to be $86 \mathrm{keV}$ $\left(10^{9} \mathrm{~K}\right)$ because the X-ray spectrum suggests the presence of components with the temperature this high. This structure was intended to model a nest of loops in an active region. Having adopted this geometry, DD showed that a formula due to Brown (1974) could be used to derive the differential emission measure, a power-1aw in $\mathrm{T}$, which would be required to produce a power-1aw $X$-ray spectrum with a given $\gamma$. The two parameters, $\alpha_{T}$ and $\alpha_{B}$, were then derivable from the hard $\mathrm{X}$-ray power-1aw spectral index, $\gamma:$.

$$
\begin{aligned}
& \alpha_{\mathrm{T}}=6 /(2 \gamma-3), \text { and } \\
& \alpha_{\mathrm{B}}=\left(7 \alpha_{\mathrm{T}} \alpha_{\mathrm{L}}-24 \alpha_{\mathrm{T}}-\alpha_{\mathrm{L}}+22\right) /\left(18-9 \alpha_{\mathrm{L}}\right),
\end{aligned}
$$

where it should be noted that $\alpha_{\mathrm{L}}$ was called $m$ in the notation of DD.

This analysis, was only applicable for events in which a power. law was an acceptable fit to the $X$-ray spectrum, and for $\alpha \mathrm{T}<2$, which corresponds to $\gamma>3$. A source with $\alpha_{T}>2$ is one in which the temperature falls off very 
rapidly with distance outside the core producing relatively little emission there. Hence, the source can be considered to be uniform at a single temperature, $T_{\max }$. The model of $\mathrm{DD}$, then, is only realistic for $0.1<\alpha \mathrm{T} \leq 2$, and $0.1<\alpha_{B}$.

In order to predict the frequency dependence of the microwave flux, the variation in the area of the source with frequency was found. Because the optical depth, $\mathrm{d}_{f}$, depends strongly on the temperature and the magnetic field, radiation at any given frequency comes primarily from a very thin layer near the level at which $d_{f}=1$. The radius, $r_{1}$, of this layer varies with frequency according to the following relation derived by $\mathrm{DD}$ :

$$
\left.r_{1} \propto f^{-10 /\left(7 \alpha_{T}\right.}+9 \alpha_{B}-1\right) \text {. }
$$

The area of emission in Equation (6) was assumed to be circular, so that we can use Equation (19) to obtain the following frequency dependence of the source area, A:

$$
A=\pi r_{1}^{2}=A_{0}\left(f_{o} / f\right)^{\alpha_{A}}
$$

where

$$
\alpha_{A}=20 /\left(7 \alpha_{T}+9 \alpha_{B}-1\right)
$$

Support for this model comes from recent observations with the VLA indicating that the area of emission commonly decreases with increasing frequency, sufficiently strongly that spectra with $\alpha_{\mathrm{L}}$ much smaller than 2 (even $\alpha_{L}<0$ ) can be explained (Marsh and Hurford 1982). A magnetic structure in which the high-frequency microwave radiation originates in smaller, Lower and probably denser areas of increased magnetic field strength is consistent with this situation.

We can now express the frequency dependence of the microwave flux by taking into account the frequency dependence of the area of emission through 
Equation (20) and of the temperature through Equations (16) and (19).

The resulting expression is as follows:

$S \propto A_{0} T_{\max } f^{\alpha} \mathrm{L}$,

where.

$$
\alpha_{L}=\left(24 \alpha_{\mathrm{T}}+18 \alpha_{B}-22\right) /\left(7 \alpha_{\mathrm{T}}+9 \alpha_{\mathrm{B}}-1\right) \text {. }
$$

Again, note that this analysis is realistic only for $\alpha_{T}$, $\alpha_{B}$ in the range from approximately 0.1 to 2 .

Following the procedure described in part III of $\mathrm{DD}$, we can calculate oT (Equation 17), $\alpha_{B}$ (Equation 18), $B_{\max }$ (Equation 9 with $\mathrm{T}_{\mathrm{x}}$ replaced by $\mathrm{T}_{\max }$ ), and $r_{\min }$ (Equations 7 with $r_{\text {peak }}$ replaced by $\cdot r_{\min }$ ). $T_{\max }$ is assumed to be 86 keV. The derived electron density of each source was calculated using the following expression given by DD and adjusted for the present system of units:

$$
\mathrm{n}^{2}=6.8 \alpha_{\mathrm{T}} \mathrm{K}_{\gamma} 50^{\gamma} \mathrm{T}_{\max }^{-3 / \alpha^{\circ}} \mathrm{r}_{\min }^{-3}\{\mathrm{r}(\gamma-1)\}^{-1}
$$

where $\Gamma$ is the gamma function.

Examination of Table $9(\$ 4.2)$ reveals that the hemispherical model developed by DD is applicable to only 7 of the 13 events at the time of maximum microwave flux. These are events $1,4,7,10,11,12$, and 13 , the events for which a power law is an acceptable fit to the X-ray spectrum. In Table 11 we present the observed parameters of these 7 GMBs and the source parameters derived from the model of DD. We also have included in Table 11 the maximum plasma $\beta$ derived using Equation (13). Events 7 and 11 have values of $\alpha_{\tilde{I}}$ which are incompatible with the model $(>2)$; absorption effects are required in this model to explain the spectrum (see \$5.3). Events 1 and 13 cannot be explained because of negative values of $\alpha_{B}$, inconsistent with the assumed behavior of $B$. Only events 10 and 12 can be explained fully with this approach. In Event 4 , the part of the spectrum with $\alpha_{\mathrm{L}}=3.1$ 
must be attributed to absorption, but the part for which $\alpha_{\mathrm{L}}=1.7$ can be explained. Events 10 and 12 are unusual because of their large values of $\alpha_{A}, 2.9$ and 2.6 , respectively. For example, event 12 was observed to produce a constant $\alpha_{\mathrm{L}}$ from

TABLE 11: DULK \& DENNIS MODEL TEST

\begin{tabular}{c|c|c|c|c|c|c|c|c|c|c} 
EVENT & $\gamma$ & $\alpha_{\mathrm{I}}$ & $\alpha_{\mathrm{T}}$ & $\alpha_{\mathrm{B}}$ & $\alpha_{\mathrm{A}}$ & $\mathrm{K}_{\gamma}$ & $\mathrm{r}_{\min }$ & $\mathrm{n}$ & $\mathrm{B}_{\max }$ & $\beta_{\max }$ \\
\hline \begin{tabular}{c|c|c|c|c|c|c|c|c|c} 
NUMBER \\
1
\end{tabular} & 3.5 & 1.4 & 1.5 & $-0.13 \dagger$ & - & - & - & - & - & - \\
7 & 4.5 & 1.7 & 1.5 & 0.8 & 1.2 & 4.2 & 4.6 & 6.3 & 120 & 0.45 \\
10 & 4.5 & $2.7 \dagger$ & - & - & - & - & - & - & - & - \\
11 & 3.1 & 0.4 & 0.94 & 0.12 & 3.0 & 0.45 & 12 & 0.16 & 24 & 0.29 \\
12 & 4.3 & 0.8 & 1.1 & 0.09 & 2.7 & 3.2 & 3.6 & 4.5 & 120 & 0.012 \\
13 & 3.1 & 1.2 & 1.9 & $-1.2 \dagger$ & - & - & - & - & - & - \\
\hline
\end{tabular}

$\dagger$ Indicates a value inconsistent with this parametric approach.

* $\alpha_{\mathrm{L}}$ applies to $3<\mathrm{f}<10 \mathrm{GHz}$, only.

1.5 to $10 \mathrm{GHz}$. If this model applies, the microwave source at $1.4 \mathrm{GHz}$ was - 140 times as large in area as at $10 \mathrm{GHz}$. There is no report of such a drastic change in source size with frequency, but no observations of source size are available at such widely-separated frequencies for a single event. This type of analysis could be compared with microwave images at we1l-separated frequencies to decide whether the model is compatible with observations.

of the events that can be explained by the model of $\mathrm{DD}$, only event 12 is also explained by the single-temperature model (Table 10). In the DD model, the derived density $n$ is a factor of 4 smaller, the value of $B_{\max }$ is 2.4 times smaller and $B$ is -7 times smaller. These differences are due to the 
choice of $\mathrm{T}_{\max }=86>\mathrm{T}_{\mathrm{X}}=27$.

It may be that an inappropriate geometry is assumed in the multi-thermal model of $\mathrm{DD}$. Optically-thick radiation is assumed to be emitted from a continuum of single-temperature sources with peak frequency and peak flux which decreases as the distance from the central core increases. Thus, such a model is inherently limited to adjusting only one spectral slope, either the low side or the high side of the microwave spectrum, but not both simultaneously. Gradients in T and B could be used, however, to explain the spectrum both above and below the peak frequency. In $\$ 5.4$, therefore, we examine a two-component, diverging loop model in which separate parts of the loop explain the low- and high-frequency portions of the microwave spectrum. In this geometry, the peak flux decreases with distance from the region of maximum temperature, but the peak frequency increases below and decreases above the altitude of temperature maximum.

\subsection{Flares for which $\alpha_{L}>2$}

Two of the events (No. 4 and No.7) exhibit a low-frequency spectral index, $\alpha_{\mathrm{L}}$, significantly larger than 2 (cf. Table 5).' For an opticallythick thermal source, this must be due to an absorption process operating outside the source (Wiehl, 1980). There are three possible absorption processes that could be operating: the Razin effect, gyroresonance absorption, and free-free absorption.

To decide which absorption process might be involved, we calculate the frequency below which each process is operative. The Razin effect (also known as medium suppression) attenuates the flux below

$$
\mathrm{f}_{\mathrm{R}}=2 \mathrm{n} / \mathrm{B}_{\perp}
$$

(Ginzburg \& Syrovatskii 1965) while gyroresonance absorption is important 
below $f_{G}=4 f_{B}$, where $f_{B}=2.8 \times 10^{-3} B$ is the gyrofrequency (Ramaty and Petrosian 1972). Free-free absorption is important below $f_{F F}$ given by the following expression: .

$$
f_{F F}=810^{-3} \mathrm{~nL}^{0.5} \mathrm{~T}^{-0.75}
$$

where the numerical constant is uncertain by $\pm 25 \%$ (Ramaty and Petrosian 1972). Values of these limiting parameters are given in Table 12 for the - two events with $\alpha_{\mathrm{L}}>2$. The frequency, $\mathrm{f}_{\mathrm{A}}$, denotes the highest frequency for which $\alpha_{L}>2$. In order to calculate $f_{G}, f_{R}$ and $f_{F F}$ we have derived $A_{\text {peak }}, B$ and $n$ as in $\$ 5.1$, making use of the fitted $T_{x}$. We have then set $L \approx r_{\text {peak }}$. For free-free absorption, we give in Table 12 both the value of $\mathrm{f}_{\mathrm{FF}}$ assuming coronal temperatures $\left(2 \times 10^{6} \mathrm{~K}\right.$ or $\left.0.17 \mathrm{keV}\right)$ and, also the value of the temperature, $\mathrm{T}_{\mathrm{FF}}$, assuming $\mathrm{f}_{\mathrm{FF}} \doteq \mathrm{f}_{\mathrm{A}}$.

TABLE 12. ABSORPTION PROCESSES

\begin{tabular}{|c|c|c|c|c|c|c|}
\hline \multirow[t]{2}{*}{$\begin{array}{r}\text { EVENT } \\
\text { NUMBER }\end{array}$} & \multirow[t]{2}{*}{$\alpha_{L}$} & $f_{A}$ & $f_{R}$ & $f_{G}$ & $\begin{array}{l}\left(\mathrm{T}=2 \times 10^{6} \mathrm{~K}\right) \\
\mathrm{f}_{\mathrm{FF}}\end{array}$ & \multirow{2}{*}{$\begin{array}{l}\mathrm{T}_{\mathrm{FF}} \\
\left(10^{6} \mathrm{~K}\right)\end{array}$} \\
\hline & & \multicolumn{4}{|c|}{$(\mathrm{GHz})$} & \\
\hline 4 & 3.1 & 4 & 0.1 & 2.4 & 0.7 & 0.2 \\
\hline 7 & 2.7 & 3 & 0.1 & 3.3 & 1.6 & 1.0 \\
\hline
\end{tabular}

Based on this analysis of the possible absorption processes that could explain values of $\alpha_{L}>2$, we conclude that

1) The Razin effect is not important in either of the two events since $f_{\mathrm{R}} \ll \mathrm{f}_{\mathrm{A}}$ in both cases.

2) Gyroresonance absorption is the most probable process responsible for values of $\alpha_{L}>2$ in these two events since $f_{G}-f_{A}$ in both cases. 
3) Free-free absorption can be ruled out for event number 4 since $T_{F F}$ calculated with $f_{F F}=f_{A}$ is significantly lower than typical coronal temperatures. Free-free absorption cannot be ruled out, however, for event number 7 since $\mathrm{T}_{\mathrm{FF}}$ is of the same order as coronal temperatures.

\subsection{A Diverging Loop Mode1 for the Microwave Source}

In this section we study a diverging loop model which might explain the observed microwave spectrum, both the low frequency and the high-frequency part. We assume that the emission at any particular frequency is dominated by the optically-thick radiation at that frequency (Matzler 1978). The crosssection of the loop increases rapidly with height above the chromosphere up to the region of maximum temperature, and then increases more gradually with altitude above that level. This behavior of the cross-section is believed to be characteristic of the leg of the loop near the footpoint (Spruit 1981). We further assume that magnetic flux is conserved along the loop. The strongest field is found near the footpoint of the loop where it approaches sunspot values $\approx 1000 \mathrm{G}$, and the lowest field near the top. Because microwave radiation at high frequencies originates from regions of high magnetic field, there exists an inverse relationship between the surface area of the emission region and the frequency. Such a frequency-area relationship is commonly observed (Marsh and Hurford 1982). To make the calculations simple, we assume the same power-1aw form for the area as in Equation (20), but now we have the following two values of $\alpha_{A}$ : $\alpha_{A 1}$ corresponding to the region above the temperature maximum and $\alpha_{\mathrm{A} 2}$ to the region below the temperature maximum.

Because the radiation at a frequency, $f$, originates mainly from the region in which the optical thickness $d_{f}=1$, the temperature of that region is 
derived from the expression for the peak frequency (Dulk and Marsh 1982), solved for $\mathrm{T}$ and adjusted for the present units:

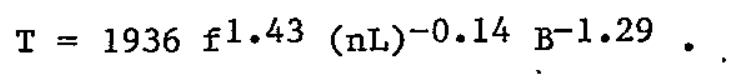

Assuming conservation of magnetic flux for a diverging flux tube and a frequency dependence of the source area as in Equation (20), we find

$$
T \propto f^{\left(1.43-1.29 \alpha_{A}\right)}
$$

The radiation below the peak frequency originates, from areas above the temperature maximum. In order to have a localized temperature maximum, a positive correlation between temperature and frequency is required in this part of the 1oop. According to Equation (28) this constrains the fanning parameter $\alpha_{A 1}<1.11$. Observations of spatially reșolved microwave sources at two or more frequencies in the range 5 to $15 \mathrm{GHz}$ indicate $\alpha_{\mathrm{A}} \approx 1$ (Marsh and Hurford 1982). This value is consistent with the above calculated range for $\alpha_{A}$ on the low-frequency. side of the spectrum.

At altitudes below the temperature maximum, we require an anticorrelation between $T$ and $f$, and this then restricts $\alpha_{A 2}>1.11$ in this region.

With the aid of Equations (16), (20) and (28) we find

$$
\mathrm{S} \propto \mathrm{f}^{\alpha} \quad \text { where } \alpha=3.43-2.29 \alpha_{\mathrm{A}}
$$

The fanning parameters, $\alpha_{A 1}$ and $\alpha_{A 2}$, are each obtained from Equation (29)

with $\alpha$ set to $\alpha_{\mathrm{L}}$ and $\alpha_{\mathrm{H}}$ respectively. Thus

$$
\begin{aligned}
& \alpha_{\mathrm{Al}}=\left(3.43-\alpha_{\mathrm{L}}\right) / 2.29 \text { and } \\
& \alpha_{\mathrm{A} 2}=\left(3.43-\alpha_{\mathrm{H}}\right) / 2.29 .
\end{aligned}
$$

Table 13 gives the values of $\alpha_{A 1}$ and $\alpha_{A 2}$ obtained for a11 13 events. (Again, events 4 and 7 cannot be explained without absorption effects - cf. §5.3). Only for event number 10 is $\alpha_{\mathrm{Al}}>1.11$ which would result in 
$T \propto \mathrm{f}^{-0.13}$. This is only a small violation of the required positive corre1ation. For a11 other events we find $\alpha_{\mathrm{A} 1}<1.11$ and $\alpha_{\mathrm{A} 2}>1.11$, insuring a decrease of the temperature with increasing distance from the region of maximum : temperature.

For $\alpha_{A}=1.5$ we obtain $\alpha_{L}=0$, corresponding to a flat spectrum, sometimes observed during microwave bursts (Hachenberg and Wallis 1961). $\because \div$

TABLE 13

\begin{tabular}{l|rrrrrrrrrrr}
$\begin{array}{l}\text { EVENT } \\
\text { NUMBER }\end{array}$ & 1 & 2 & 3 & 5 & 6 & 8 & 9 & 10 & 11 & 12 & 13 \\
\hline$\alpha_{\mathrm{A} 1}$ & 0.89 & 0.62 & 0.89 & 1.10 & 1.02 & 0.76 & 0.58 & 1.32 & 0.58 & 1.15 & 0.97 \\
$\alpha_{\mathrm{A} 2}$ & 3.46 & 2.24 & 1.76 & 2.11 & - & 3.11 & 1.72 & 1.93 & 1.89 & 2.33 & - \\
\hline
\end{tabular}

In the lower part of the loop the temperature must decrease rapidly with increasing frequency and, therefore, must increase with altitude above the photosphere. 'For $\mathrm{T} \propto \mathrm{f}^{-2}, \mathrm{~T}_{\max } \approx 70 \mathrm{keV}$. At the lower extremity of the source, there must be a high-frequency break in the spectrum; above this frequency the source is optically-thin, and the spectrum is steep ( $\alpha^{-8}$ ). Assuming that the peak frequency is $15 \mathrm{GHz}$ and the high frequency break is at $35 \mathrm{GHz}$, we find the temperature of the $35 \mathrm{GHz}$ level to be approximately 13 $\mathrm{keV}$, and, by using Equation (9), we obtain $B \approx 1700 \mathrm{G}$. This value is consistent with observations of photospheric fields obtained for the 13 events which varied between 1100 and $2500 \mathrm{G}$ (Coffey, 1980, 1981).

By assuming a simple diverging loop model in which the lower part fans out more rapidly than the upper part, we are able to explain the observed microwave spectra in terms of two fanning parameters, $\alpha_{\mathrm{A} 1}$ and $\alpha_{\mathrm{A} 2}$. 
The values of $\alpha_{\mathrm{Al}}$ are in accord with commonly observed source properties. The values of $\alpha_{\mathrm{A} 2}$ are, however, higher than the observed rate of change of area with frequency reported from direct imaging measurements. These values of $\alpha_{A 2}$ are consistent with the observations of Kundu et al. (1982) in that they attribute significant emission to regions of relatively low altitudes as well as the loop legs above the temperature maximum. Observations of dynamic microwave spectra obtained in coincidence with microwave images over the same range of frequencies would provide a more critical test of this diverging Ioop model. 


\section{CORRELATIONS}

In searching for a possible relationship between any pair of parameters $(x, y)$, we first obtained a new pair $(\log x, \log y)$ and then tested for evidence of a linear correlation between the two logarithms. Correlation coefficients, $r$, between the various pairs of observed and derived parametérs have been calculated together with the probability, $P_{c}(r, N)$, that any random sample of uncorrelated data points would yield a linear correlation coefficient greater than or equal to the value obtained for $r$ (Equation 7.8 of Bevington 1969). All correlation coefficients. (r) refer to this linear dependence in $\log -\log$ space of $\mathrm{N}$ data points. Because the number of data points, $N$, is not the same for each of the parameter pairs, the probability, $P_{c}(r, N)$, rather than the correlation coefficient, $r$, is the figure of merit for the significance of the correlations. All those pairs for which $\mathrm{P}_{\mathrm{C}}(\mathrm{r}, \mathrm{N}) \geqslant 40 \%$ are considered as "not correlated". Pairs for which $5 \%<\mathrm{P}_{\mathrm{c}}(\mathrm{r}, \mathrm{N})<40 \%$ are considered "correlated" and all pairs for which $\mathrm{P}_{\mathrm{c}}(r, N)<5 \%$ are "we11 correlated".

Considered as a member of this set, event No. 11 is anomolous. Not only is it less impulsive and of longer duration than all other flares in the group of 13 studied, but the ratio of its microwave to hard X-ray peak fluxes is greatest and its microwave emission is delayed the longest. Taking all the available data into account, we have chosen to regard this event as the second stage of event No. 10 and have omitted event No. 11 from the correlation analysis.

\subsection{Mode1-Independent Parameters}

In order to determine how the characteristic times, $\tau_{\mu \mathrm{c}}$, of the micro- 
TABLE 14: FREQUENCY DEPENDENCE OF THE MICROWAVE CHARACTERISTIC TIME $\tau_{\mu \mathrm{C}}$

\begin{tabular}{|c|c|c|c|c|c|}
\hline $\begin{array}{l}\text { EVENT } \\
\text { NUMBER }\end{array}$ & $\mathrm{D}_{\mu}$ & $g_{\mu}$ & $r$ & $N$ & $P_{c}(r, N)$ \\
\hline 1 & & & -0.71 & 4 & 0.28 \\
\hline 2 & 188 & -0.97 & -0.99 & 4 & 0.005 \\
\hline 3 & 59 & -0.31 & -0.94 & 5 & 0.014 \\
\hline 4 & 139 & -0.36 & -0.99 & 4 & 0.003 \\
\hline 5 & & & -0.85 & 3 & 0.35 \\
\hline 6. & 268 & -0.50 & -0.93 & 5 & 0.021 \\
\hline 7 & & & -0.94 & 4 & 0.06 \\
\hline 8 & & & -0.86 & 5 & 0.059 \\
\hline 9 & & & -0.47 & 6 & 0.33 \\
\hline 10 & & & -0.83 & 5 & 0.081 \\
\hline 11 & & & -0.60 & 5 & 0.28 \\
\hline 12 & 178 & -0.43 & -0.90 & 5 & 0.037 \\
\hline 13 & & & -0.91 & 4 & 0.088 \\
\hline
\end{tabular}

$$
\mathrm{r}=\text { correlation coefficient }
$$$$
\mathrm{N}=\text { number of points }
$$ 
TABLE 15: ENERGY DEPENDENCE OF THE HARD X-RAY CHARACTERISTIC TIME $\tau_{\mathrm{Xc}}$

\begin{tabular}{|c|c|c|c|c|c|}
\hline $\begin{array}{l}\text { EVENT } \\
\text { NUMBER }\end{array}$ & $\mathrm{D}_{\mathrm{x}}$ & $g_{x}$ & $r$ & $\mathrm{~N}$ & $\mathrm{P}_{\mathrm{c}}(r, \mathrm{~N})$ \\
\hline 1 & 3160 & -0.56 & -0.96 & 9 & $<0.001$ \\
\hline 2 & 40 & -0.31 & -0.98 & 8 & $<0.001$ \\
\hline 3 & 29 & -0.21 & -0.93 & 10 & $<0.001$ \\
\hline 4 & 130 & -0.35 & -0.98 & 10 & $<0.001$ \\
\hline 5 & 142 & -0.31 & -0.98 & 10 & $<0.001$ \\
\hline 6 & 566 & -0.53 & -0.98 & 10 & $<0.001$ \\
\hline 7 & 169 & -0.40 & -0.89 & 8 & 0.0028 \\
\hline 8 & 74 & -0.40 & -0.88 & 10 & $<0.001$ \\
\hline 9 & 719 & -0.57 & -0.97 & 10 & $<0.001$ \\
\hline 10 & 361 & -0.32 & -0.89 & 9 & 0.0012 \\
\hline 11 & 581 & -0.10 & -0.82 & 12 & 0.0012 \\
\hline 12 & 113 & -0.37 & -0.86 & 10 & 0.0014 \\
\hline 13 & 46 & -0.28 & -0.87 & 8 & 0.0051 \\
\hline
\end{tabular}

$r=$ correlation coefficient, $N=$ number of points 
wave bursts depend on the frequency, we performed such a correlation analysis for each GMB. Table 14 shows that a power-law relation of the form $\tau_{\mu c}=$ $D_{\mu} f^{g}$ for the frequency dependence of $\tau_{\mu c}$ is a valid description in 5 of the 13 cases (i.e. $P_{c}(r, N) \leqslant 5 \%$ ). In Figure 14 we plot the values of $\tau_{\mu \mathrm{c}}$ as a function of frequency for all 13 events. Straight lines indicate cases in which a power law approximation is valid. Even in those cases for which this approximation is not acceptable at the $5 \%$ confidence level, there is a general decrease of $\tau_{\mu c}$ with increasing frequencies. Similar relationships exist for the rise and fall times of the microwave evetns, $\tau_{\mu \mathrm{r}}$ and $\tau_{\mu \mathrm{f}}$.

The characteristic time, $\tau_{x c}$, for the hard $X$ rays also exhibits a power-law energy dependence of the form

$$
\tau_{\mathrm{Xc}}=\mathrm{D}_{\mathrm{x}} \mathrm{E}^{\mathrm{g}_{\mathrm{x}}} \text {. }
$$

We found a correlation of $\tau_{x c}$ with the mean photon energy at a confidence level of $P_{c}(r, N)$ better than $0.5 \%$ in all 13 events (Table 15). The time ${ }^{T_{X C}}$, is a measure of the burst duration. The values of $g_{X}$ are in the range from -0.56 to -0.10 , the average being -0.35 . In Figure 29 the measured values of $\tau_{x c}$ are shown as a function of photon energy together with the correlation curves. Similar relationships hold for the rise and fall times, as well. For the rise time $\tau_{x r}$, the average exponent was -0.5 with only 7 events correlated at the $1 \%$ confidence level. For the fall time $\tau_{\mathrm{Xf}}$, the average exponent was -0.4 with al1 but one event correlated at the $5 \%$ confidence level.

In Table 16 we summarize the results of the correlation analysis of the time parameters of the hard $X$-ray and microwave bursts. The hard $X$-ray bursts exhibit à good correlation between rise and fall times at all energies. 
TABLE 16

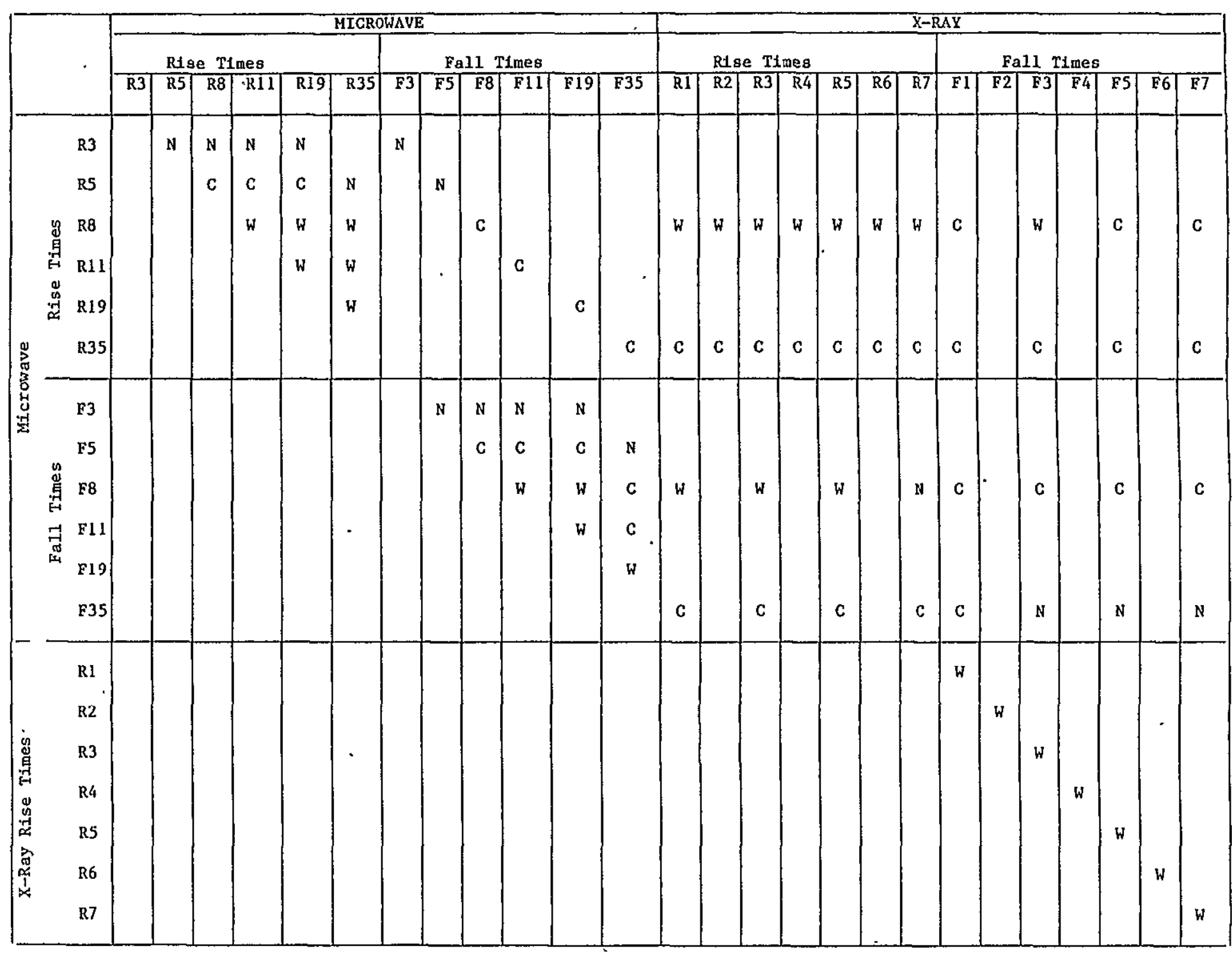

" $R$ " Indicates rise time, "F" Indicates fall time. For the microwave times", the numbers refer to frequency in CHz; for the X-ray times, the numbers indicate channel number. The goodness of the correlation is indicated by " $N$ " for pairs which are not correlated $(P>40 \%)$, by " $C$ " for correlated pairs $(5 \%<P<40 \%$ ) and by "W" for well correlated pairs $(\mathrm{P}<5 \%)$. 
The microwave bursts exhibit a less significant correlation between rise and fall times, and there is evidence for a second component at low frequencies which is not correlated with the bursts at high frequencies.

We have searched for correlations between other measured and derived parameters and summarize below the most important findings of the correlation analysis.

The microwave rise and fall times at all frequencies are not correlated with the corresponding maximum flux at any particular frequency. We also computed the rate of increase RI, defined as the maximum microwave flux at a given frequency divided by the rise time at that frequency, and the rate of decrease $\mathrm{RD}$, defined as the maximum flux at a given frequency divided by the fall time at that frequency. It was found that the values of RI, are well correlated with the values of $\mathrm{RD}$, at all microwave frequencies except at $3 \mathrm{GHz}$. The hard $\mathrm{X}$-ray peak $\mathrm{flux}$ is not correlated with the microwave flux at the peak frequency or with the maximum flux at $11 \mathrm{GHz}$. The transition frequency, $f_{t}$, is correlated with the peak frequency, confirming a result found by Guidice and Castelli (1975). However, the high frequency cutoff, $f_{h}$, is not correlated with the peak frequency. Also, a measure of the width of the microwave spectrum, $f_{h}-f_{t}$, is not correlated with $f_{\text {peak }}$. As mentioned in $\$ 4$, at $t_{\mu \max }$ a power-law function was an acceptable representation of the X-ray spectrum for only 7 of the events. However, the best fit $\gamma$ can be used to parameterize the hardness of the spectrum even when the fit is not acceptable, provided that the values of $\gamma$ are regarded only as rough indicators of the spectral hardness. If the microwave source electrons are the same as (or closely related to) the X-ray source electrons, some relation between $\gamma$ and the microwave spectral parameters is expected. The ex- 
cellent microwave and $X$-ray spectral coverage available in this study enabled us to search for such correlations for the first time. It was found that, at $t_{\mu \max }$, the values of $\gamma$ and $f_{\text {peak }}$ are correlated at the $6 \%$ confidence level, a correlation that has not been reported previously. The relation is $f_{\text {peak }} \propto \gamma^{-2.6}$. The expected correlation between $\gamma$ and $\alpha_{\mathrm{H}}$ is not found, however; we discuss possible explanations in $\$ 7$.

No correlation was found between $g_{x}$ and the microwave spectral parameters $f_{\text {peak }}, \alpha_{H}, \alpha_{L}$ and $s_{\text {peak, }}$, when all 13 events were considered. However, when attention was limited to events $1,2,3,8,12$ and 13 , which had $\mathrm{X}$-ray spectra that were well represented by single-temperature bremsstrahlung functions, it was found that $g_{X}$ and $\alpha_{H}$ were correlated at the $1 \%$ confidence level and that $g_{X}$ and $f_{\text {peak }}$ are correlated at the $9 \%$ confidence level. For this subset of the events, $f_{\text {peak }}$ and $\alpha_{H}$ are also correlated at the $0.6 \%$ confidence leve1.

\subsection{Mode1-dependent Parameters}

We first discuss the results of tests for correlations involving parameters derived assuming a single-temperature source. A thermal energy density, ED, defined by equation (11), was derived from each of events 1, 2, 3, 8,12 and 13 , the events for which a single-temperature bremsstrahlung function was an acceptable fit to the $x$-ray spectrum at $t_{1 \max }$. The values of ED and $g_{x}$ given by equation (31) were correlated at the $0.3 \%$ confidence leve1. We found $\mathrm{g}_{\mathrm{x}} \propto \mathrm{ED}^{-0.58}$. The parameter $\mathrm{g}_{\mathrm{X}}$ and the density, $\mathrm{n}$, were correlated at the $0.7 \%$ confidence level with $g_{x} \propto n^{-0.25}$. A peak was also correlated with $\mathrm{g}_{\mathrm{X}}$, at the $6 \%$ confidence level. Other parameters derived in a single-temperature mode1 $(B, U, B)$ are not correlated with $g_{x}$. Both ED and $A_{\text {peak }}$ were correlated with ofi to better than $1.5 \%$ con- 
fidence. There were slight correlations of $B$ and $\beta$ with of $_{\text {, }}$, to $10 \%$ and $15 \%$ confidence, respectively.

As noted in $\$ 4$, at $t_{\mu \max }$, a thermal function was not an acceptable representation of the $\mathrm{X}$-ray spectrum for 6 of the 13 events. However, the best fit $E M$ and $T$ can be used to parameterize the spectrum even when the fit is not acceptable, as long as their values are regarded only as rough indicators of the intensity and hardness of the spectrum. The curve in the EM-T plane which is generated as the spectrum evolves in time is a useful constraint on any thermal model that is proposed to explain the flare. Therefore, we studied the EM-T correlation for a11 13 events.

The 13 events can be grouped into 3 different classes as far as the EM-T correlation is concerned and typical examples of each class are shown in Figure 43. The first class exhibits a relatively flat EM-T correlation in which the emission measure remains approximately constant while the temperature first increases and then decreases (e.g. event 2). Six events exhibit such a behavior. The second class ( 4 events) shows anticorrelation between EM and T (represented by events 7 and 10 ). Such a relation was reported earlier for two events of 1972 May 18 (Wiehl et al. 1980). They ascribed this anticorrelation to expansion of the source. The third class (e.g. event, 12) can be described by a positive correlation between EM and $\mathrm{T}$ ( 3 events). The power-law index of $T$ in this correlation is different from the value $3 / 2$ which would be expected from the adiabatic model (Matzler et al. 1978). Also, these curves exhibit a considerable amount of hysteresis: $T$ remains large while the EM decreases during the decline of the event. Hence none of these events are compatible with a reversible, adiabatic process. 


\section{DISCUSSION}

\subsection{Mode1-independent Results}

We draw several conclusions about whether the same electrons produce both the hard $\mathrm{X}$-rays and microwaves. In $\$ 6.1$, the microwave temporal parameters $\left(\tau_{\mu r}, \tau_{\mu f}\right.$ and $\left.\tau_{\mu c}\right)$ were tested for correlations with their $X$-ray counter-parts. While the temporal parameters for frequencies above $5 \mathrm{GHz}$ are correlated from event to event with the X-ray parameters, the lowfrequency parameters are not. This may be evidence that the electrons responsible for the low-frequency emission are not the same as those that produce the $\mathrm{X}$ rays and high-frequency microwaves. The peaks in the time histories for $f<5 \mathrm{GHz}$ suggest that they are closely related, however. This difference in low-frequency time behavior may also indicate a different source region in space. The correlations of high-frequency parameters with X-ray parameters are consistent with a common source.

The correlation of $\gamma$ and $f_{\text {peak }}(\$ 6.1)$ is evidence that the same electrons produce the $X$ rays and the microwaves near $f_{\text {peak }}$. This is expected whether a thermal or nonthermal interpretation is made (cf. Dulk \& Marsh 1982). A correlation between $\gamma$ and $\alpha_{H}$ is to be expected if the electron spectrum dominates the microwave spectrum, but this is not found. The fact that the high-frequency temporal parameters and $\mathrm{X}$-ray temporal parameters are correlated, but the spectral parameters are not suggests that gradients in the magnetic field of the microwave emission region dominate the highfrequency portion of the microwave spectrum.

Next we consider the relationships among the time of maximum microwave flux, $t_{\mu \max }$, the time of maximum $x$-ray flux, $t_{x \max }$, and the time of 
hardest $X$-ray spectrun, $t_{x h}$. All of the $X$-ray events exhibited a softhard-soft spectral evolution, implying that a corresponding evolution of the source electron distribution occurred. The microwave emissivity of an electron distribution increases rapidly with the relative number of high-energy electrons. Hence, if the same electron distribution is the source of both hard $x$-rays and microwaves, it is to be expected that $t_{\text {maxax }}=t_{x h}$. This is often not the case (cf. Table 9). B also has a strong effect on the microwave emissivity, however (Dulk and Marsh 1982). The difference between $t_{x h}$ and $t_{\mu \max }$ can be explained by changes in $B$ during the event. On the average, $t_{\mu \max }$ is closer to $t_{x h}$ than to $t_{x \max } \cdot$.

The events that had X-ray spectra which were fit acceptably by a thermal function seem to represent a distinct class of flares in other respects. For these 6 events, $f_{\text {peak }}$ was correlated with $g_{X}$, the parameter which characterized the variation of $\tau_{x c}$ with energy. A value of $g_{X}$ near -1 corresponds to an event in which there was a large change in $\gamma$ during the event, while $g_{X} \approx-0.1$ indicated that $\gamma$ was relatively constant. The $X$-ray spectral evolution in each event followed a soft-hard-soft pattern; therefore, if $\left|g_{x}\right|$ was sma11, the $X$-ray spectrum was relatively hard for a relatively longer time during the event. The events with small $\left|g_{x}\right|$ tended to have higher $f_{\text {peak }}$, in agreement with expectations but why the correlation of $g_{X}$ and $f_{\text {peak }}$ breaks down when the spectrum does not fit a thermal function at $t_{\mu \max }$ is not apparent.

We have observed that the $\mathrm{X}$-ray characteristic time, $\tau_{x c}$, decreases with increasing $X-$ ray energy. Similar observations motivated a complex theoretical investigation of the dependence of X-ray fall time on energy, assuming a source distribution of nonthermal electrons in a trap of nonuniform density 
(Brown 1972). It should be noted, however, that in a wide variety of models the hard $\mathrm{X}$-ray spectrum evolves with a soft-hard-soft time behavior (hardest near the time of maximum flux) so that the events exhibit a decline in characteristic time with energy. A power-law dependence of the event duration with energy is, therefore, not a sensitive test for a trap model. To illustrate this, we briefly consider one of many possible models with soft-hard-soft spectral evolution resulting in a power-law dependence of characteristic time on energy: adiabatic compression. In our example, the hard X-ray emitting . plasma is heated at a constant rate from 10 to $60 \mathrm{keV}$ in 5 seconds and the emission measure reaches a peak of $10^{45} \mathrm{~cm}^{-3}$. The process is then reversed. In Figure 44 we show the fraction of maximum $f l u x\left(I(t) / I_{\max }\right)$ versus time. for five photon energies in the HXRBS range. The characteristic times, $\tau_{x c}$, also plotted in Figure 44 are determined by the same method as in our analysis of these 13 events (see Equation (4)). It is obvious from Figure 44 that $\tau_{x c}$ is shorter, the higher the energy. Comparing Fig. 44 with 29, we see that the observed energy dependence of $\tau_{x c}$ is simply a soft-hard-soft spectral behavior. All of these events do exhibit a soft-hard-soft spectral evolution. This could result from a variety of properties of the heating or acceleration mechanism, and does not point unambiguously to a trap model.

\subsection{Mode1-dependent Results}

In $\$ 5.1$ we derived source parameters for the 6 events for which a single-temperature interpretation of the X-ray spectrum was possible, obtaining values which are reasonable for the active region corona. Nonuniformities in $B$, which would then be needed to. explain the full microwave spectra, were ignored, and only $f_{\text {peak }}$ and $s\left(f_{\text {peak }}\right)$ were used in the derivation. In one instance (Event 12) the derived source area was available from a direct meas- 
urement of the source size. In the time interval from 0658:52 to 0659:02 UT which included $t_{\mu \max }, t_{x \max }$ and $t_{x h}$, an image of the region was obtained at X-ray energies from 17 to $40 \mathrm{keV}$ with the SXT instrument onboard the Hinotori spácecraft. Ohki et al. (1982) reported that the $\mathrm{X}$-rays originated in a single elliptical source with dimensions of approximately 10 arc sec by 30 arc sec. The area within the $40 \%$ peak flux contour is $1.2 \times 10^{18} \mathrm{~cm}^{2}$. This is consistent with our prediction of $1.3 \times 10^{18} \mathrm{~cm}^{2}$ for the source size at peak emission. This successful prediction of the source area from observations with no spatial resolution lends support to the hypothesis that there are impulsive flares in which a common single-temperature source produces both microwaves and hard $\mathrm{X}$-rays.

The correlations of ED (equation 11), $\mathrm{n}$ and $\mathrm{A}_{\text {peak }}$ (equation 7) with $g_{\mathrm{x}}$ (equation 31) also suggest that the single-temperature interpretation is physically significant for the flares involved. Smaller values of $\left|g_{x}\right|$, indicating that the $\mathrm{X}$-ray spectrum was relatively hard for a relatively long portion of the burst, are associated with relatively high values of energy density, ED, and vice versa.

Both the multi-thermal model of $\mathrm{DD}$ and the diverging loop model developed in $\$ 5.4$ were consistent with the properties of some events. Both models require changes in source size with frequency which can be investigated in future multi-frequency imaging observations. The effects of the geometry and nonuniformities of the magnetic field configuration on the spectra will be crucial to understanding flares. 


\section{CONCLUSION}

Our study of 13 Great Microwave Bursts and associated hard X-ray events has led to the following conclusions:

- Eight of the observed microwave spectra have a constant power-law index from the peak frequency down to $1 \mathrm{GHz}$ or less. One spectrum extends to $<0.4 \mathrm{GHz}$.

- A microwave spectral index of less than 2 below the peak frequency was observed for most of the events, implying nonuniformities in the sources.

- The time of the hardest $X$-ray spectrum is delayed for most of the events relative to the time of maximum $X$-ray flux. So is the time of maximum microwave flux.

- The low-frequency microwave spectrum is explained in less than one third of the events by the simple hemispherical geometry of Dulk and Dennis (1982).

- The temporal parameters characterizing the high-frequency microwave time histories are correlated with each other and with the corresponding hard $\mathrm{X}$-ray parameters.

- The low-frequency microwave temporal parameters are not correlated either with the corresponding high-frequency microwave parameters or with the hard X-ray parameters.

- For 6 events in which a single-temperature interpretation of the X-ray spectrum was possible, the derived source density and energy density were found to be correlated with the independent measured parameter $g_{x}$ (equation 31). 
- The source area derived using a single-temperature model is in good agreement with the observed area obtained for the one event for which a spatially resolved hard $\mathrm{X}$-ray image is available.

- In thermal models, complex source structures are required to explain the complete microwave spectrum, both above and below the peak frequency.

- Correlation of the microwave peak frequency with the best fit X-ray spectral index implies that the bulk of microwave and hard X-ray emission is attributable to a single distribution of electrons. 


\section{ACKNOWLEDGMENTS}

It is a pleasure to thank $G$. Wharen for typing the often chaotic and constantly changing manuscript. We appreciate the help of H.E. Dennis, A.K. Tolbert, B.E. Gibson, G.S. Kennard, and A.J. Kiplinger in the data reduction. We thank H.E. Coffey, NOAA, and D.J. Forrest, .University of New Hampshire, for additional data, and G.A. Dulk, University of Colorado, and A. Magun, University. of Berne, for helpful discussions. This research was carried out while one of the authors (HJW) was a visiting scientist at the Laboratory for Astronomy and Solar Physics of the NASA Goddard Space Flight Center with a fellowship from the Swiss National Science Foundation. The hospitality of C.J. Crannell and K.J. Frost, SMM Project Scientist, is gratefully acknowledged. 


\section{REFERENCES}

Batchelor, D.A., Benz, A.0., and Wiehl, H.J. 1983, in preparation.

Bevington, P.R. 1969, Data Reduction and Error Analysis for the Physical Sciences (New York: McGraw-Hi11).

Brown, J.C. 1971, Solax Phys., 18, 489.

Brown, J.C. 1972, Solar Phys., 25, 158-77.

Brown, J.C. 1974, in Coronal Disturbances, G. Newkirk, ed., IAU Symp. 57, 395.

Brown, J.C. 1975, in Solar Gamma, X-, and EUV Radiation, S.R. Kane, ed., IAU Symp. 68, p. 245 .

Brown, J.C., Melrose, D.B., and Spicer, D.S. 1979, Ap.J., 228, 592.

Brown, J.C., Craig, I.J.D., and Karpen, J.T. 1980, Solar Phys., 67, 143.

Chubb, T.A., Kreplin, R.W., and Friedman, H. 1966, J. Geophys. Res., 71, 3611.

Coffey, E. 1980, SGD Comp. 433; 1980, SGD Comp. 436; 1981, SGD Comp. 437.

Coffey, H. 1981, SGD Comp. 441; 1981, SGD Comp 446; 1982, SGD Comp. $\underline{\underline{450}}$; 1982, SGD Comp. 454 .

Coffey, H. 1982, SGD Prompt., 455.

Colgate, S. 1978, Ap.J., 221, 1068.

Cranne11, C., Frost, K., Matzler, C., Ohki, K., Saba, J. 1978, Ap.J., 223, 620-37.

Datlowe, D.W. 1975, Space Sci. Inst., $\underline{\underline{1}}, 389$.

Datlowe, D.W. 1977, Nuc. Inst. \& Meth., 145, 365.

Dennis, B.R., Frost, K.J., Orwig, L.E., Kiplinger, A., Dennis, H.E., Gibson, B.E., Kennard, G.S., and Tolbert, A.K. 1983, NASA Tech Memo. 84998 .

Duijveman, A., Hoyng, P., and Machado, M.E. 1982, Solar Phys., 81, 137.

Dulk, G. and Marsh, K. 1982, Ap.J., 259, 350-8.

Dulk, G. and Dennis, B. 1982, Ap.J., 260, 875-84.

E1can, M. 1978, Ap.J. , 226, L99.

Frost, K.J. 1969, Ap.J., 158, L159. 
Ginzburg, V.L. and Syrovatskii, S.I. 1965, Ann. Rev. Astron. Astrophys., 297.

Groenschild, E.H.B.M. and Mewe, R. 1978, Astron. Astrophys. Suppl., Ser. $\underline{\underline{32}}, 283$.

Guidice, D. and Castelli, J. 1975, Solar Phys., 44, 155-72.

Hachenberg, 0. and Wallis, G. 1961, Z. Astrophys., 52, 42.

Holt, S. and Ramaty, R. 1969, Sol. Phys., 8, 119.

Hoyng, P., Machado, M.E., Duijveman, A., Boelee, A., de Jager, C., Fryer, R., Galama, M., Hoekstra, R., Imhof, J., Lafleur, H., Maseland, H.V.A.M., Mels, W.A., Schadee, A., Schrijver, J., Simnett,.G.M., Svestka, Z., van Beek, H.F., van Tend, W., van der Laan, J.J.M., van Rens, P., Werkhoven, F., Willmore, A.P., Wilson, J.W.G., and Zandee, W. 1981a, Ap.J., 244, L153.

Hoyng; P., Duijveman, A., Machado, M.E., Rust, D.M., Svestka, Z., Boelee, A., de Jager, C., Frost, K.J., Lafleur, H., Simnett, G.M., van Beek, H.F. and Woodgate, B.E. 1981b, Ap.J., 246, L155.

Hoyng, P., Marsh, K.A., Zirin, H., and Dennis B.R. 1983, Ap.J., 268, 865.

Kane, S. and Anderson, A.K. 1970, Ap.J., 162, 1003.

Kane, S.: 1972, Space Sci. Rev., $\underline{\underline{13}}, 822$.

Kane, S.R., Cranne11, C.J., Datlowe, D., Feldman, U., Gabrie1, A., Hudson, H.S., Kundu, M.R., Matzler, C., Neidig, D., Petrosian, V., Sheeley, N.R., Jr. 1980, Solar Flares, P.A. Sturrock, Ed.

Kane, S., 1982 Proceedings of the Hinotori Symp. on Solar Flares, 89-91, Jan. 27-29, Japan

Kaplan, S.A., Pikel'ner, S.B., and Tsytovich, V.N. 1974, Phys. Reports C, $\underline{\underline{15}}(1), 1$.

Kawabata, K., Ogawa, H., Takakura, T., Tsuneta, S., Ohki, K., Yoshimori, M., Okudaira, K., Hirashima, Y., Kondo, I. 1982, Proceedings of the Hinotori Symp. on Solar Flares, 168-185, Jan. 27-29, Japan.

Kundu, M.R. 1961, J. Geophys. Res., 66, 4308.

Kundu, M.R., Schmah1, E.J., and Velusamy, T. 1982, Ap.J., 253, 963.

Kundu, M.R. and Vlahos, L., 1982, Space Sci. Rev., 32, 405.

McKenzie, D. 1972, Ap.J., 175, 481-92.

Magun, A., Fuhrer, M., Kampfer, N., Stahli, M., Schochlin, W., Wieh1, H. 1981, Solar Observations Report No. 46, University of Berne. 
Marsh, K.A., Hurford, G.J., Zirin, H., Dulk, G.A., Dennis, B.R., Frost, K.J., Orwig, L.E., 1981, Ap.J., 251 797.

Marsh, K. and Hurford, G. 1982, Ann. Rev. Astron. Astrophys., 497.

Matteson, J.L. 1971, Ph. D. Thesis, UCSD, San Diego, CA.

Matzler, C., Bai, T., Crannell, C., Frost, K. 1978, Ap.J., 223, 1058-71.

Matzler, C. 1978, Astron. Astrophys., 70, 181-88.

Ohki, K., Tsuneta, S., Takakura, T., Nitta, N., Makishima, K., Murakami, T., Ogawara, Y., Oda, M., Miyamoto, S. 1982, Proceedings of the Hinotori Symp. on Solar Flares, 102-119, Jan. 27-29, Japan.

Orwig, L.E., Frost, K.J. and Dennis, B.R. 1980, Solar Phys., $\underline{\underline{65}}, 25$.

Peterson, L.E. and Winkler, J.R. 1959, J. Geophys. Res., 64, 697.

Ramaty, R., Petrosian, V. 1972, Ap.J., 178, 241.

Rust, D.M., Nelson, J.J., Pryor, L.H., Frank, Z.A., and Boggess, A.E. 1982, Solar Maximum Year Flare List, SMM Data Analysis Center Document.

Schochlin, W. and Magun, A. 1979, Solar Phys., 64, 349.

Smith, D. and Lilliequist, C. 1979, Ap.J., 232, 582-9.

Smith, D. 1980, Solar Phys., $\underline{\underline{66}}, 135$.

Spicer, D. and Brown, J. 1981, in The Sun as a Star, S. Jordan (ed.), NASA SP-450.

Spruit, H.C. 1981, in The Sun as a Star, S. Jordan, (ed.), NASA SP-450.

SGD, Explanation of data reports Feb. 1982, No. 450 Supplement.

Takakura, T. 1972, Solar Phys., 26, 151.

Takakura, T. and Kai, K. 1966, Pub1. Astron. Soc. Japan, 18, 57.

Takakura, T.: 1975, Solar Gamma, $-X$, and EUV Radiation, S.R. Kane, (ed.), . IAU Symp. 68, p. 245 .

Takakura, T., Ohki, K., Tsuneta, S., Nitta, N., Makishima, K., Murakami, T., Ogawara, Y., and Oda, M. 1982, Proceedings of the Hinotori Symp. on Solar Flares, 142-161, Jan. 27-29, Japan.

Tsuneta, S., Ohki, K., Takakura, T., Nitta, N., Makishima, K., Murakami, T., Ogawara, Y., Odo, M., and Kondo, I. 1982, Proceedings of the Hinotori. Symp. on Solar Flares, 142-161, Jan 27-29, Japan. 
Wieh1, H. 1980, Dissertation, University of Berne.

Wiehl, H., Schochlin, W., Magun, A. 1980, Astron.\& Astrophys., 92, 260-66.

Wiehl, H. and Desai, U. 1983, to appear in July Ap.J.

Wieh1, H. and Matzler, C. 1980, Astron. Astrophys., 82, 93.

Zolcinski, M.C., Forrest, D.J., Ryan, J.M., Chupp, E.I., Kanbach, G., Reppin, C., Rieger, E., and Share, G.E. 1982, presented at the 24th Plenary Meeting of COSPAR, Ottawa, Canada. 
NAMES AND ADDRESSES OF AUTHORS

D.A. Batchelor, C.J. Crannell, B.R. Dennis, and P.N. Price Code 684,

NASA Goddard Space Flight Center

Greenbe1t, MD 20771.

H.J. Wieh1

Institute for Astronomy

ETH

8092 Zurich

Switzerland 


\section{FIGURE CAPTIONS}

Figures 1 to 13

Time histories of the Great Microwave Bursts at three microwave frequencies, of the $\mathrm{X}$-ray bursts at two energies and of the best fit temperature, computed from the X-ray spectra.

\section{Figure 14}

Microwave characteristic times.as a function of frequency for all 13 events. Straight lines indicate cases in which a power-1aw approximation is acceptable. The numbers designate the individual events.

Figures 15 to 27

Microwave spectra at the time of maximum microwave, emission. Open circles - indicate Berne data while closed circles are additional data from SGD Comprehensive Reports (Coffey, 1980-82).

\section{Figure 28}

Polarization as a function of frequency as observed with the Berne instruments. $L$ and $R$ indicate left handed and right handed polarization, respectively. The numbers next to the points designate the individual events.

\section{Figure 29}

Hard X-ray characteristic times as a function of energy, determined using channels 1 to 9 . Straight lines indicate acceptable power-law fits. The numbers designate the individual events. Data points and fits for events 3,8 and 12 are multiplied by factors of 100,10 and 0.1 , respectively, to separate them from the results of other events.

$$
-64-
$$


Figures 30 to 42

Hard X-ray spectra at the time of maximum microwave intensity for both power-1aw and thermal assumptions of the incident spectra. Horizontal bars through the points in these plots indicate the channel widths and vertical bars indicate the statistical uncertainties.

\section{Figure 43}

Correlation diagrams between emission measure (EM) and temperature (T) for four events. The dashed dotted lines indicate the general behaviour of the correlation. In each case the event begins at the point with the lowest temperature.

\section{Figure 44}

Plots of the rising part of the X-ray time profile computed for several different energies assuming adiabatic compression. The time history for each energy is normalized to its peak value. The following part of the time history is the reverse of the rising part for a reversible adiabatic process. The inset shows the characteristic times, $\tau_{\mathrm{xc}}$, computed from the indicated time profiles plotted as a function of energy in the HXRBS range. 
Figure 1

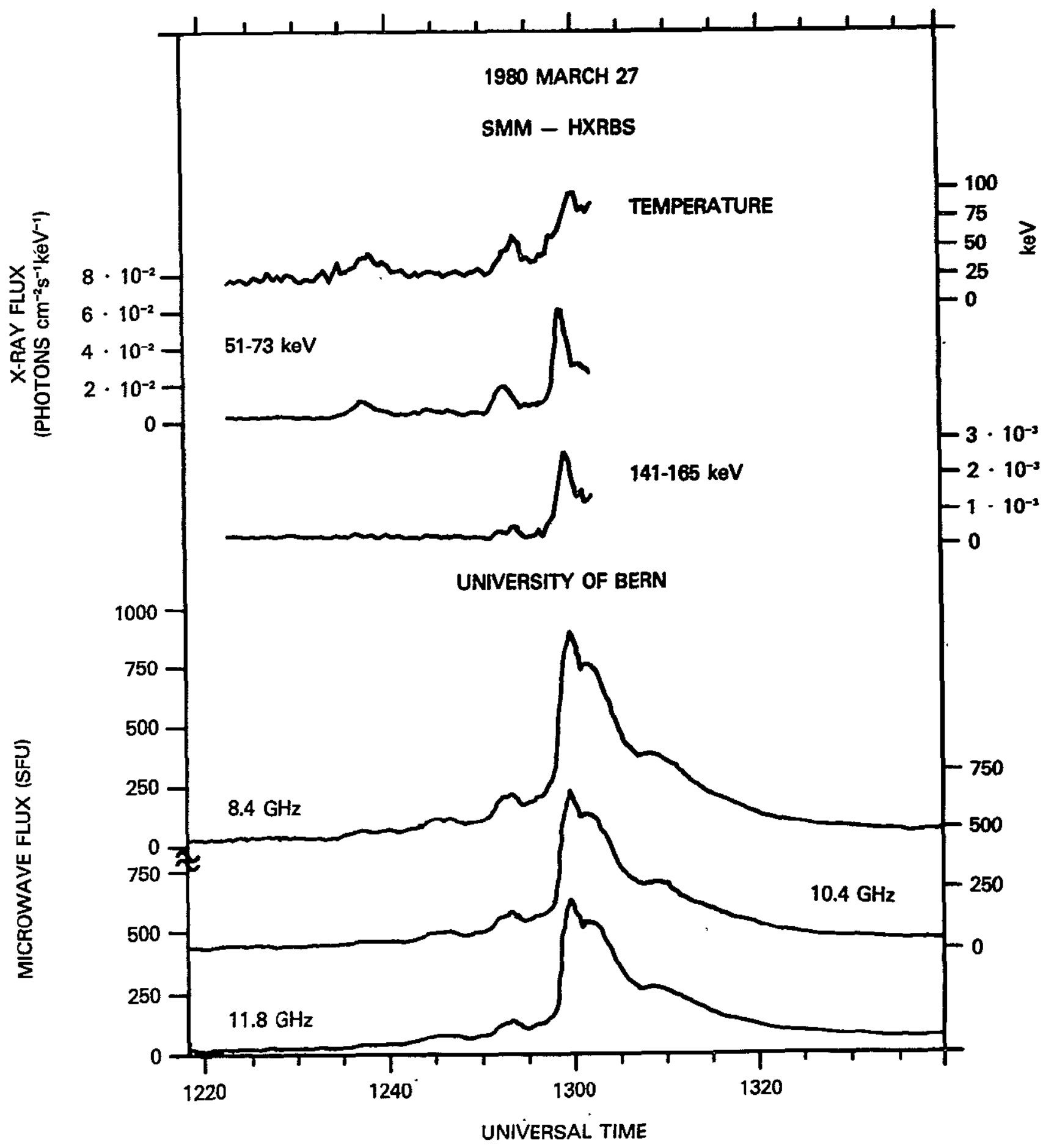


Figure 2

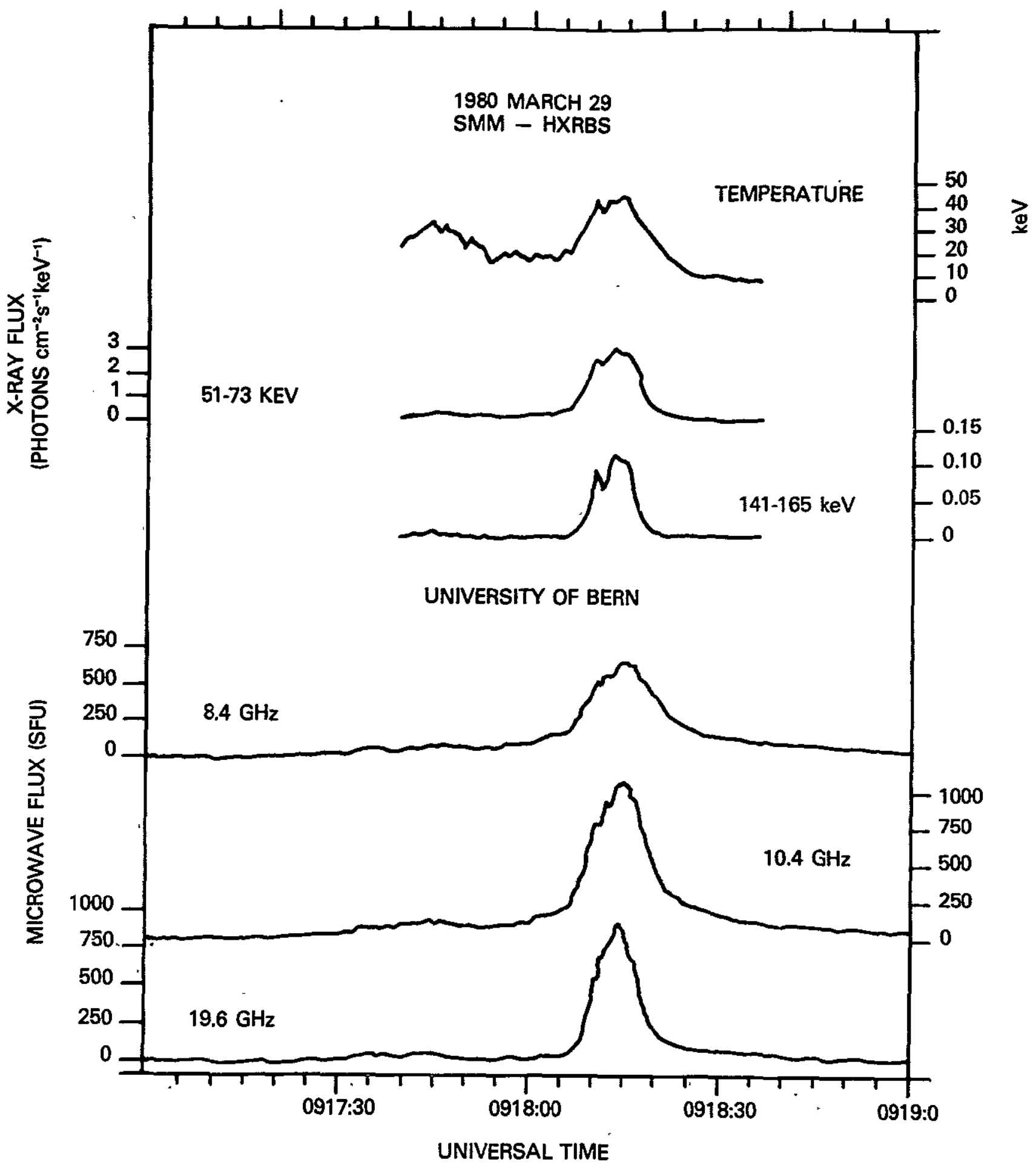


Figure 3

ORIGINAL PAO른 OF POOR QUALITY

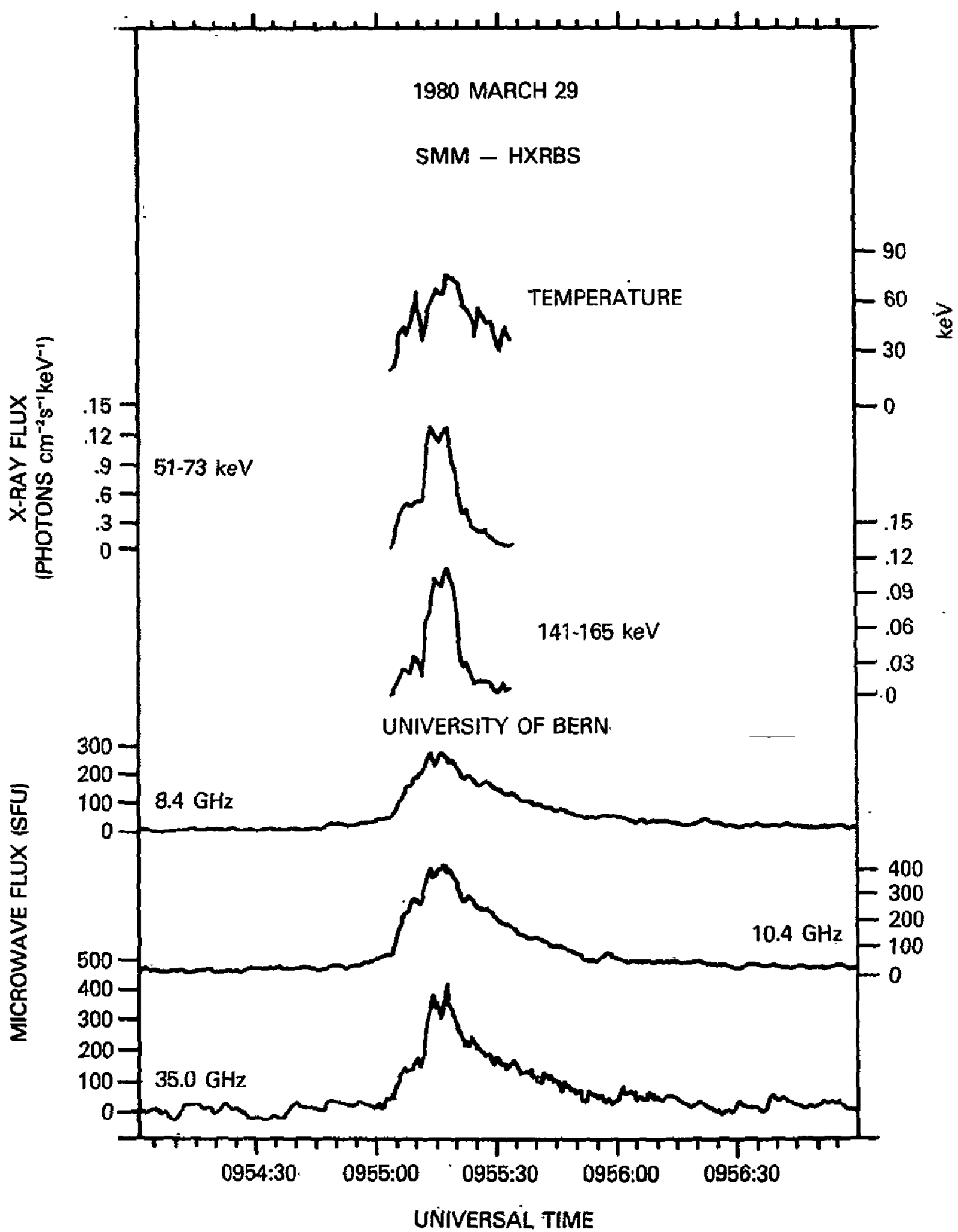




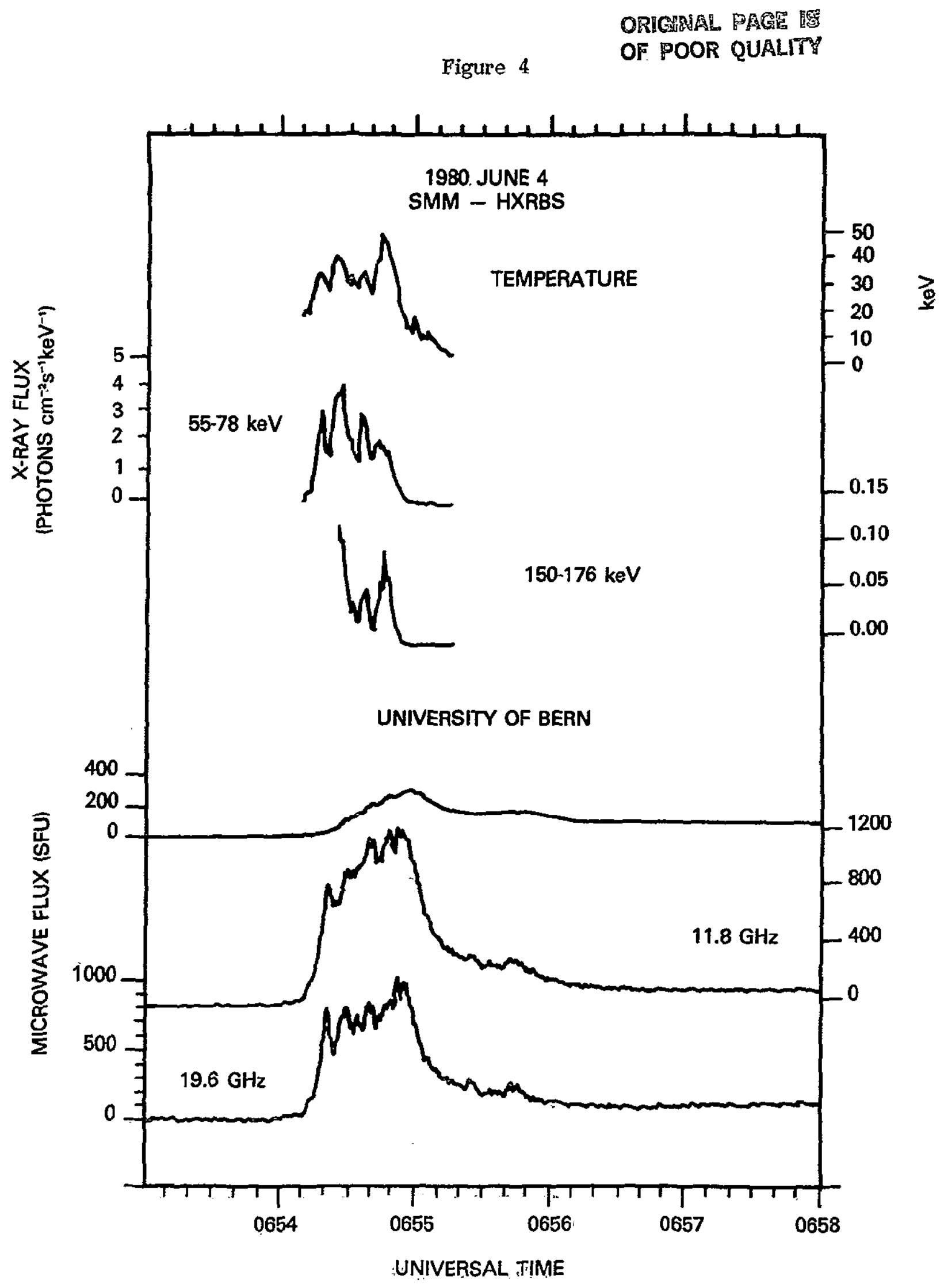


Figure 5

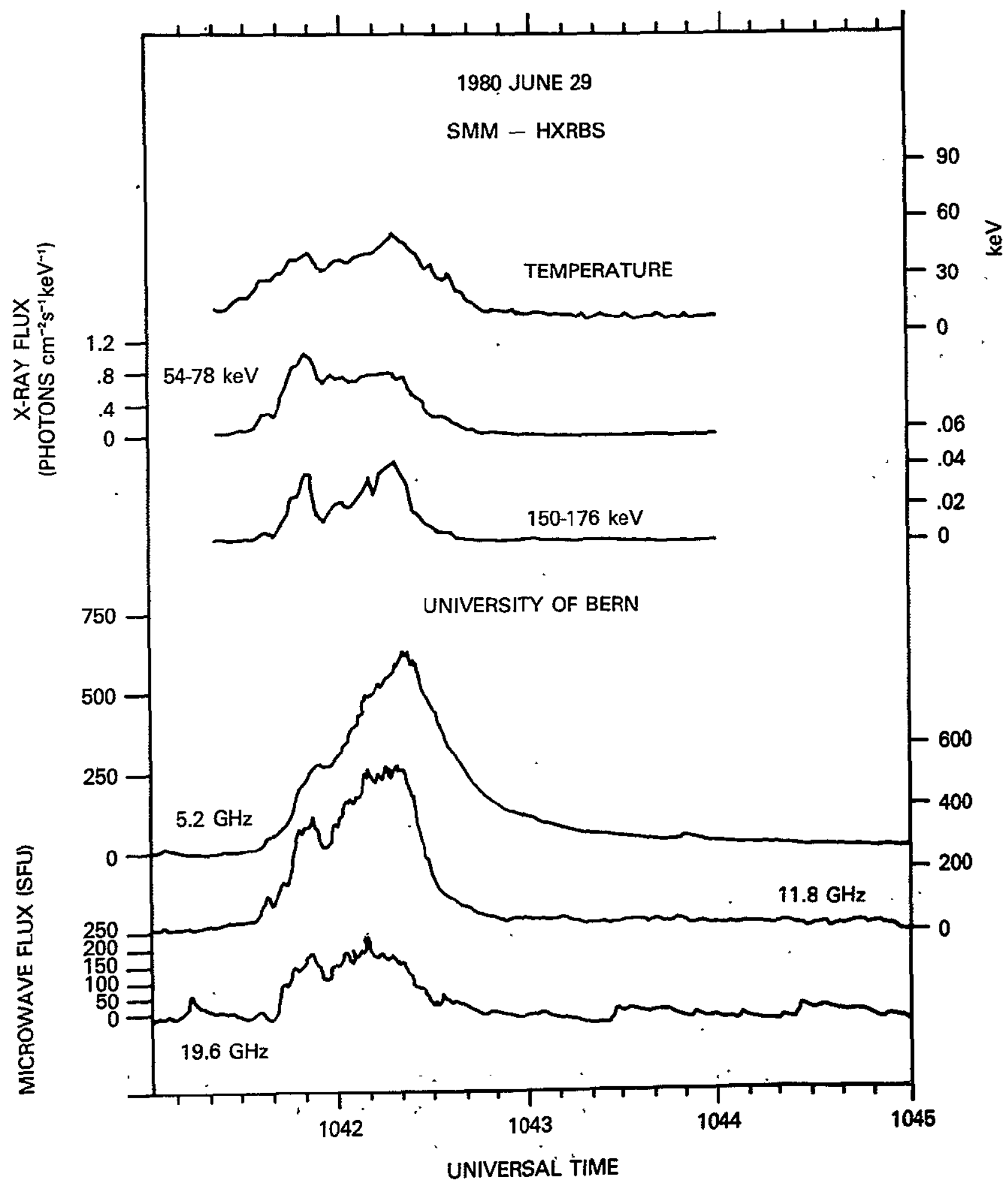


Figure 6

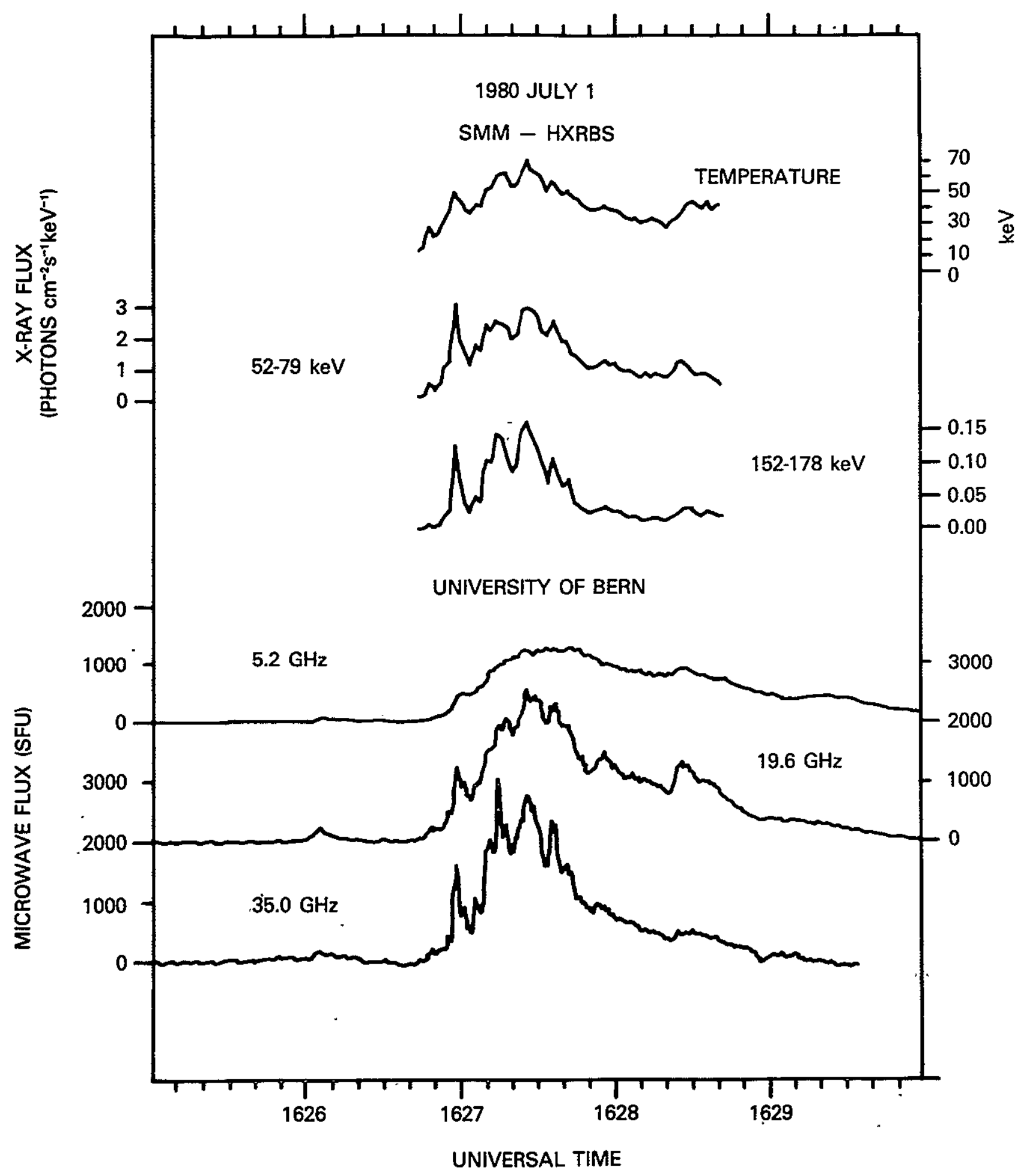




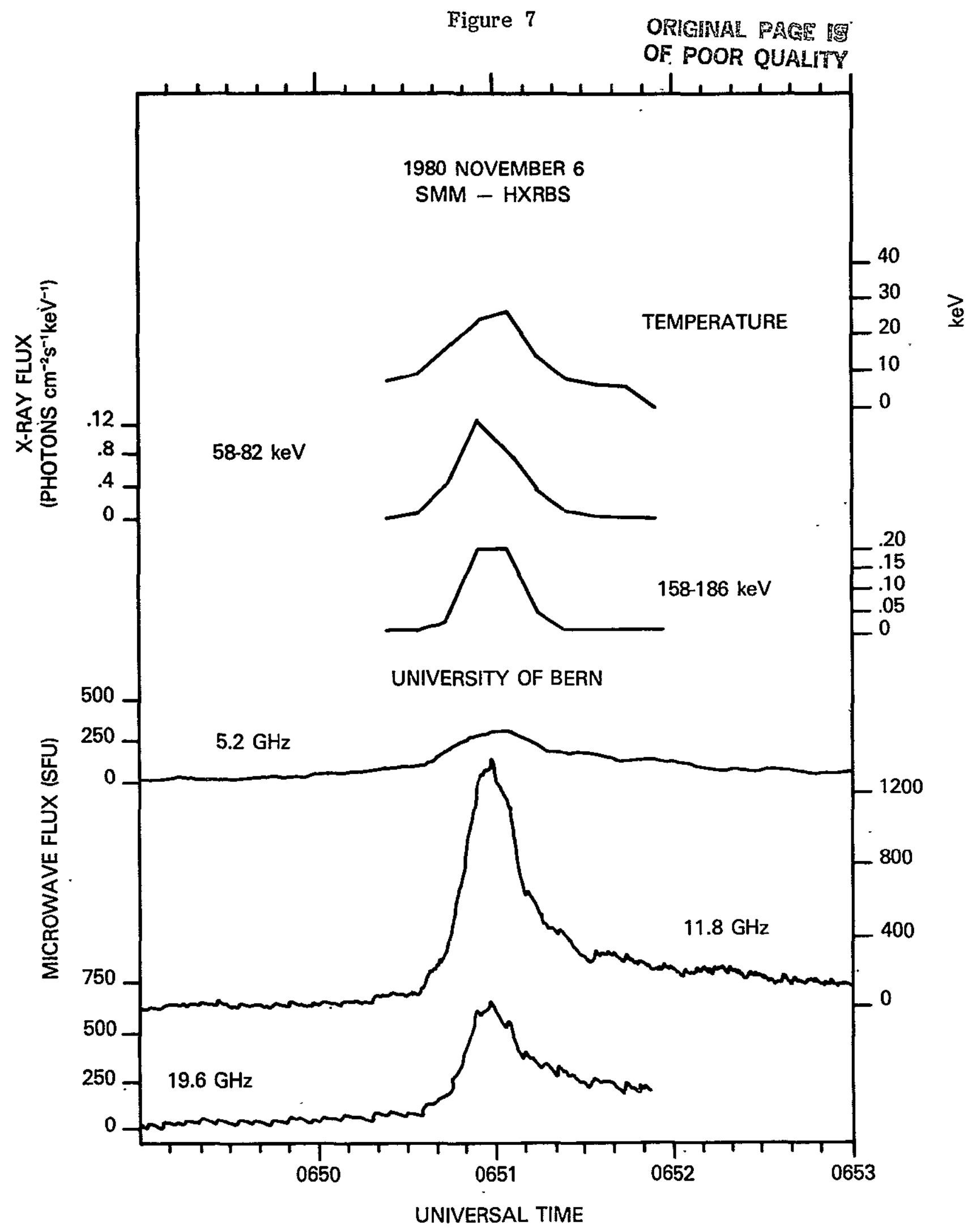




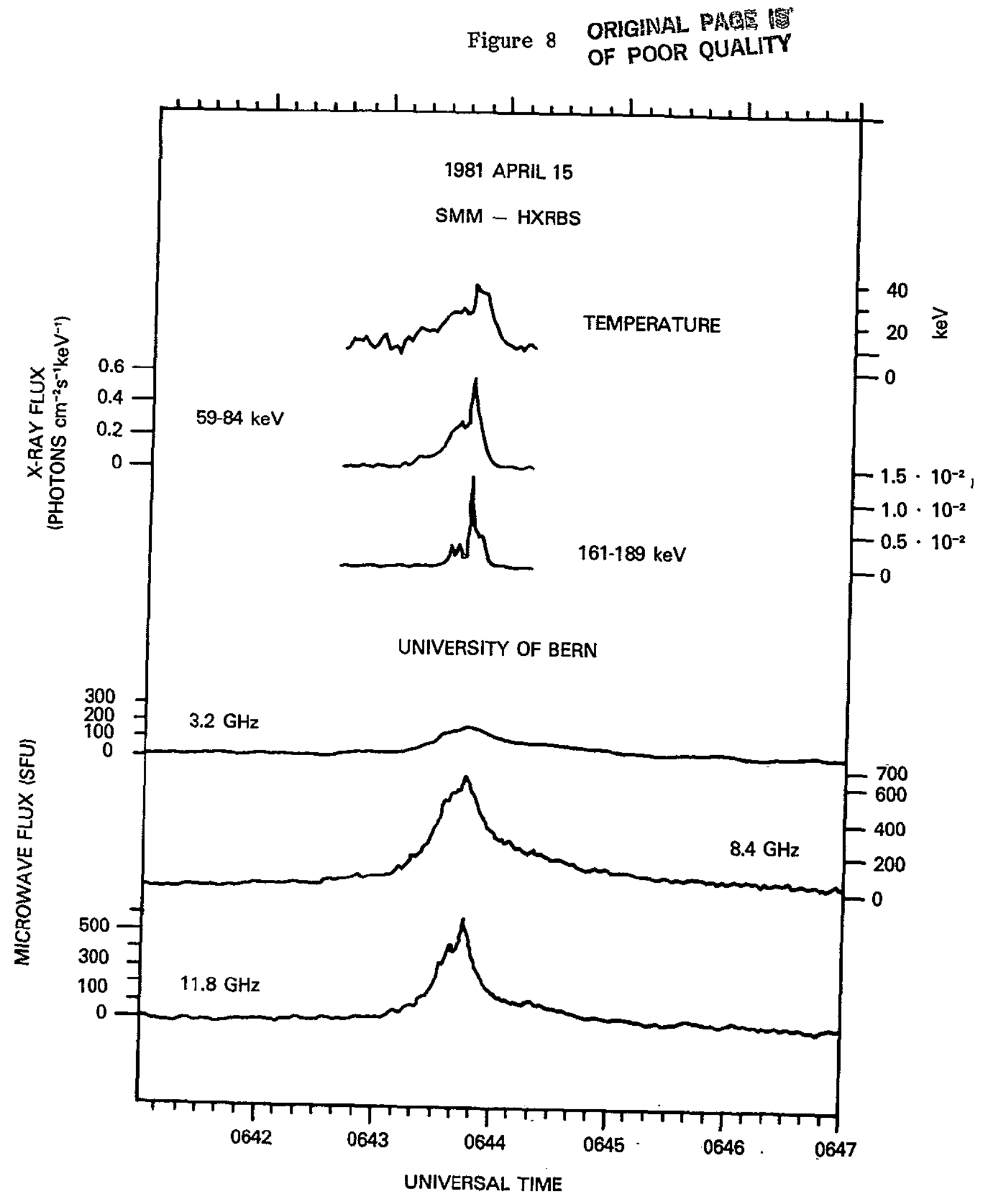




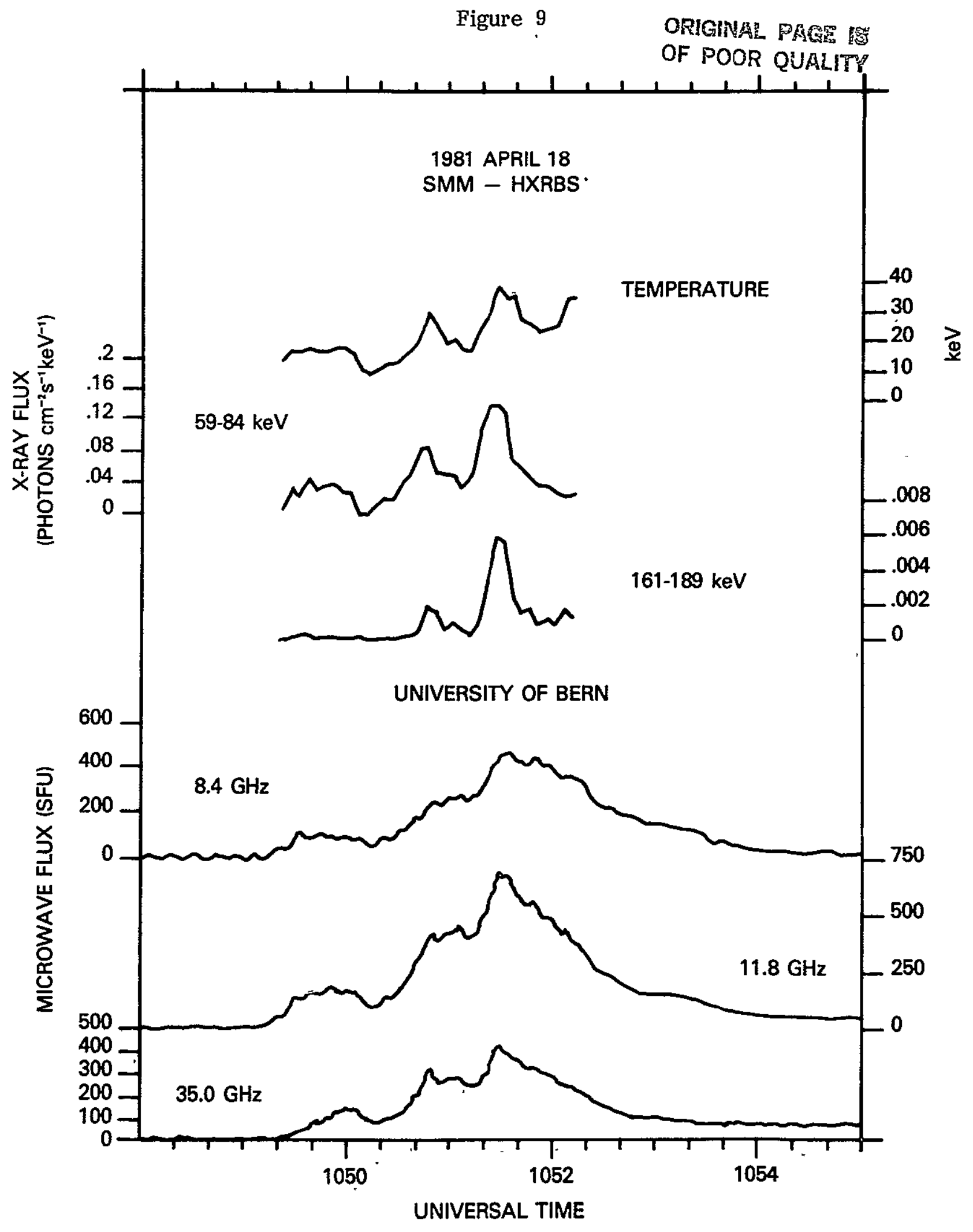




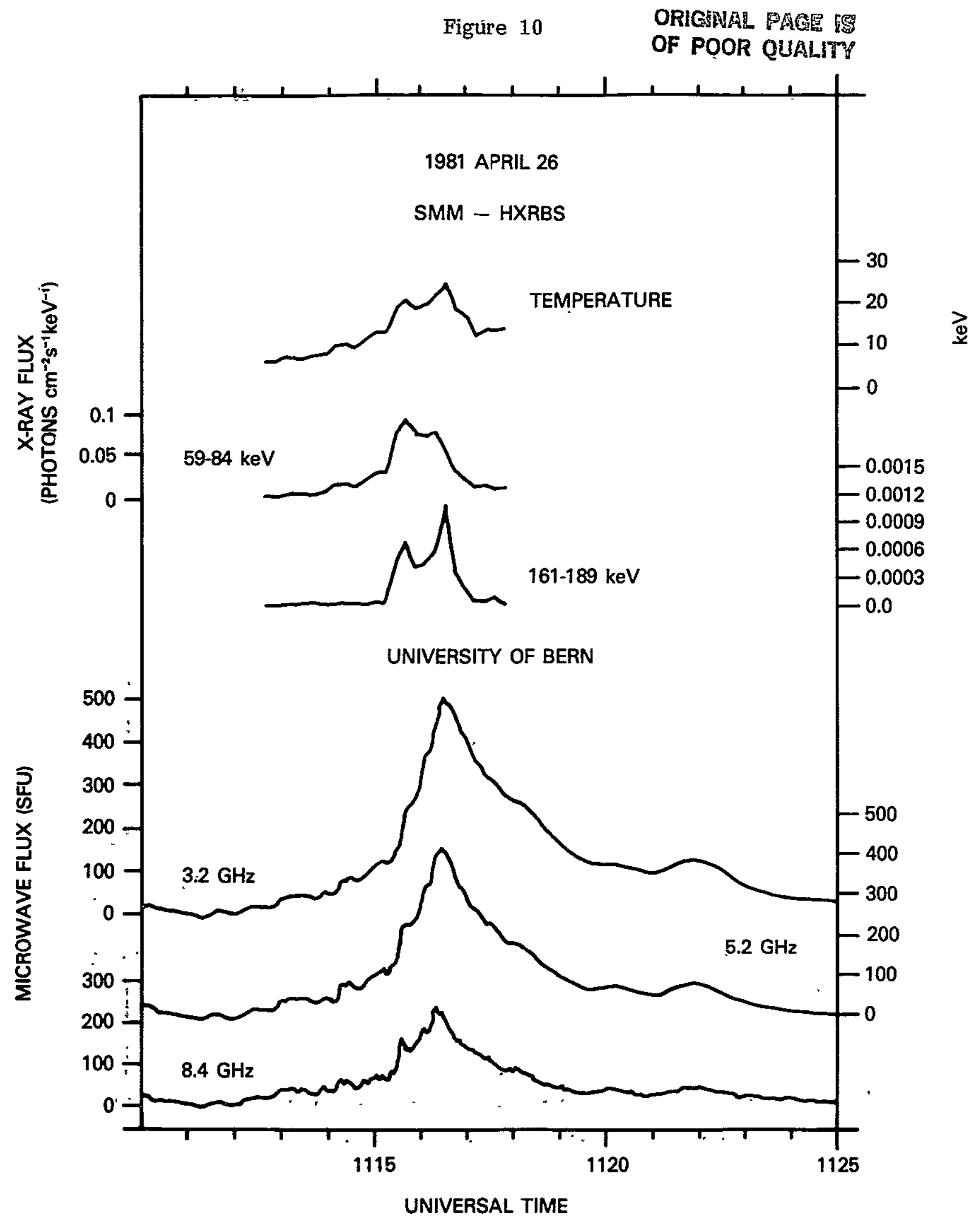




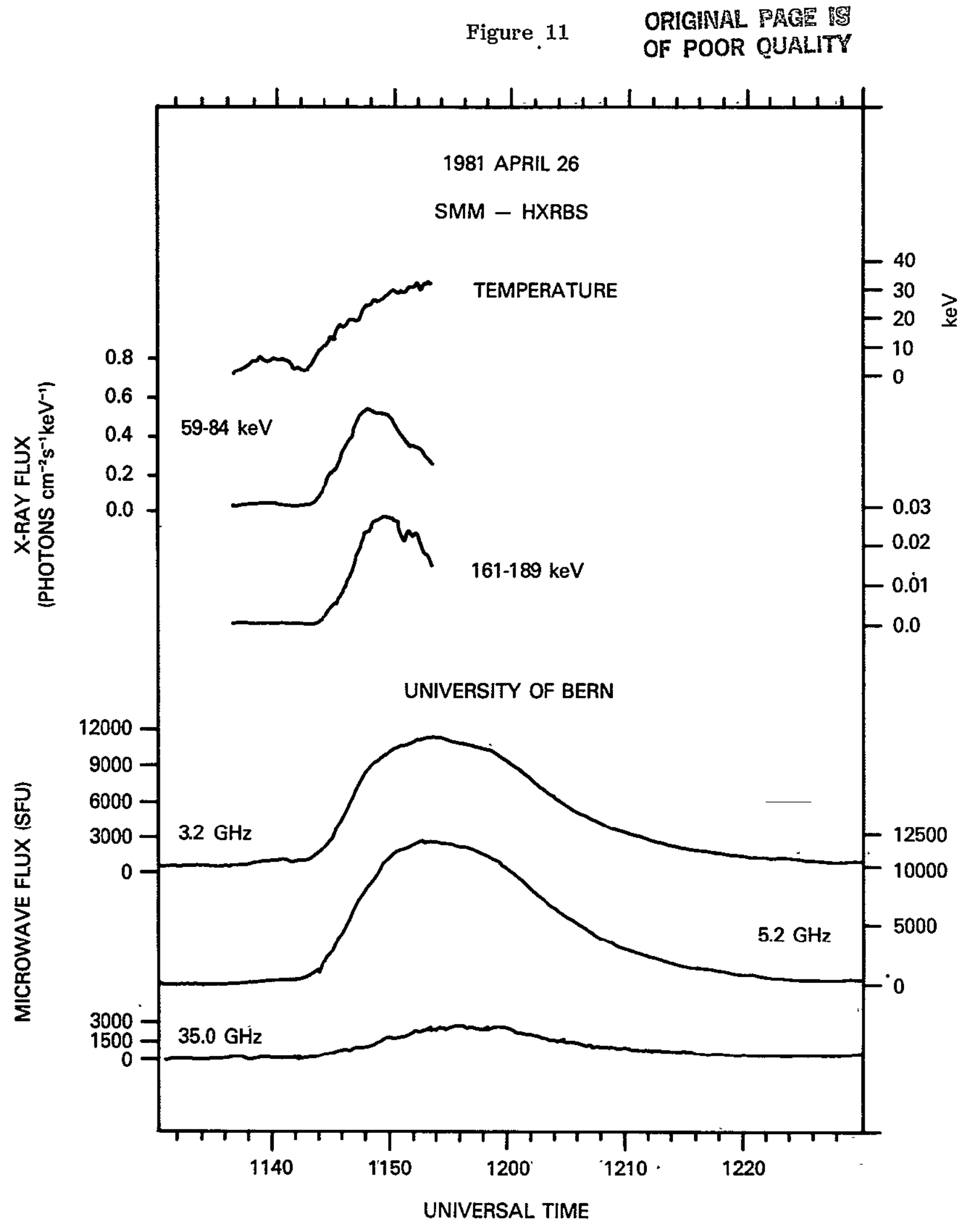


$\begin{array}{ll}\text { ORIGINAL PAOE } & \text { OF } \\ \text { Figure. } 12 & \text { OF POOR QUALITY }\end{array}$

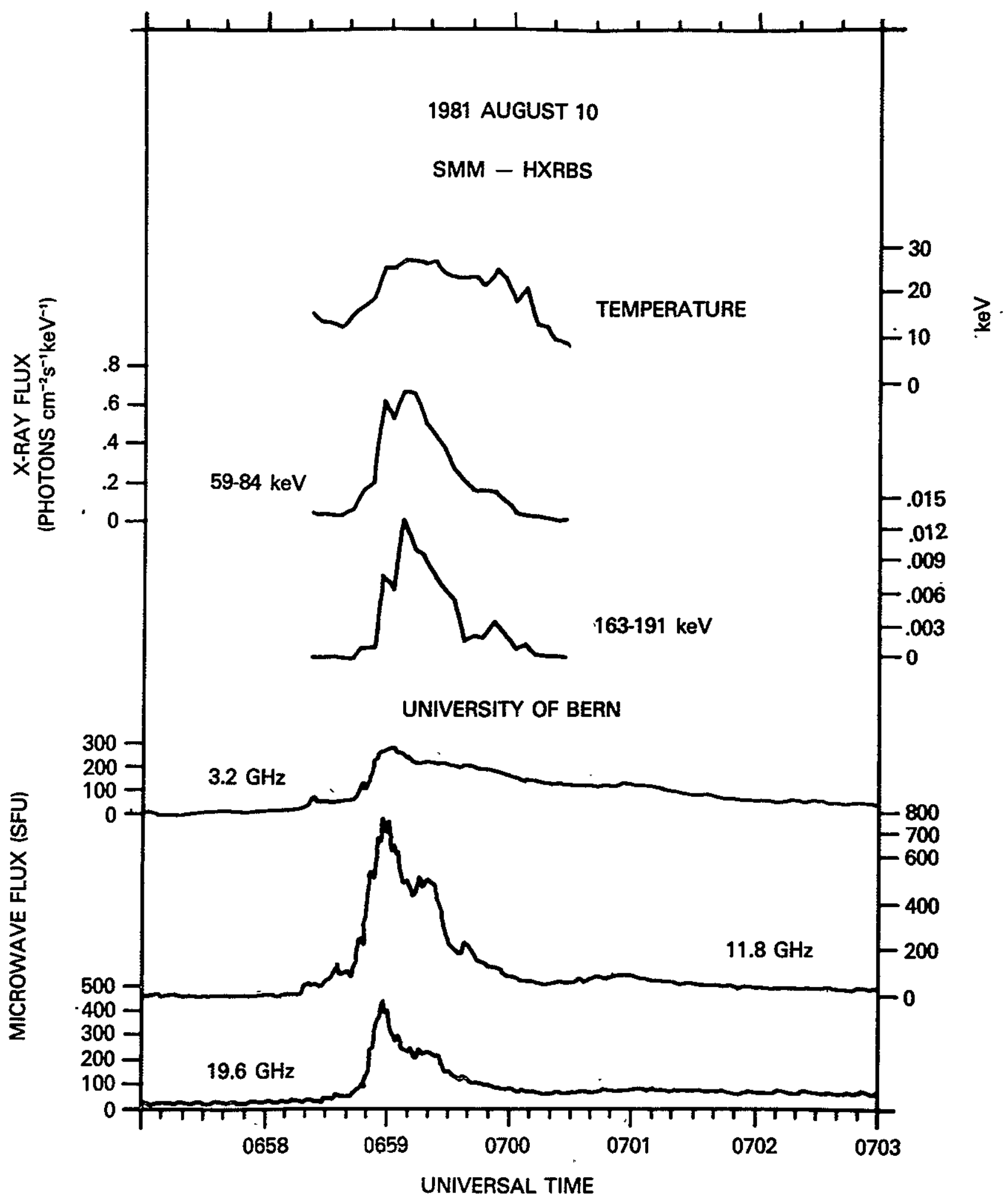


Figure 13

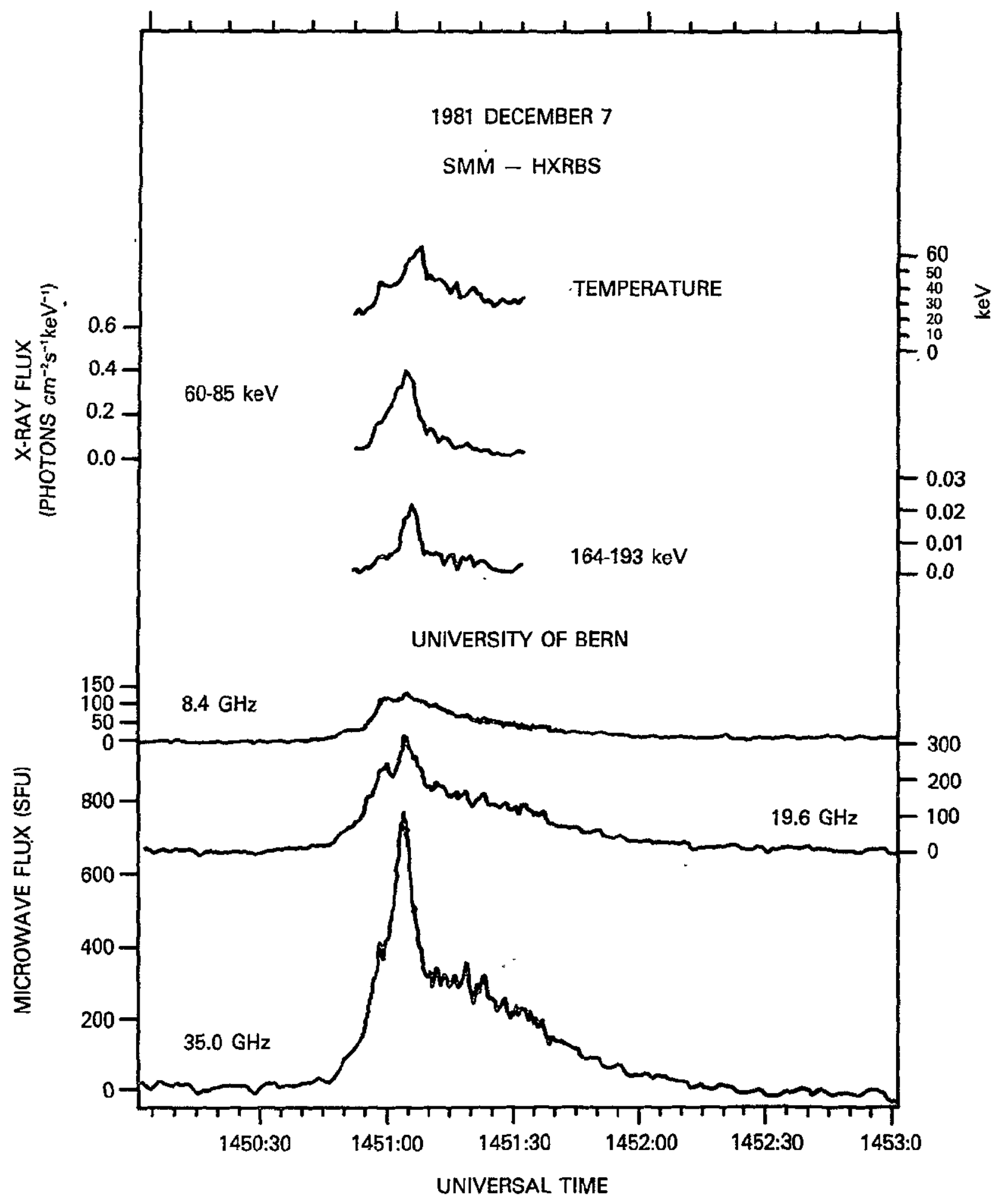




\section{MICROWAVE CHARACTERISTIC TIME}

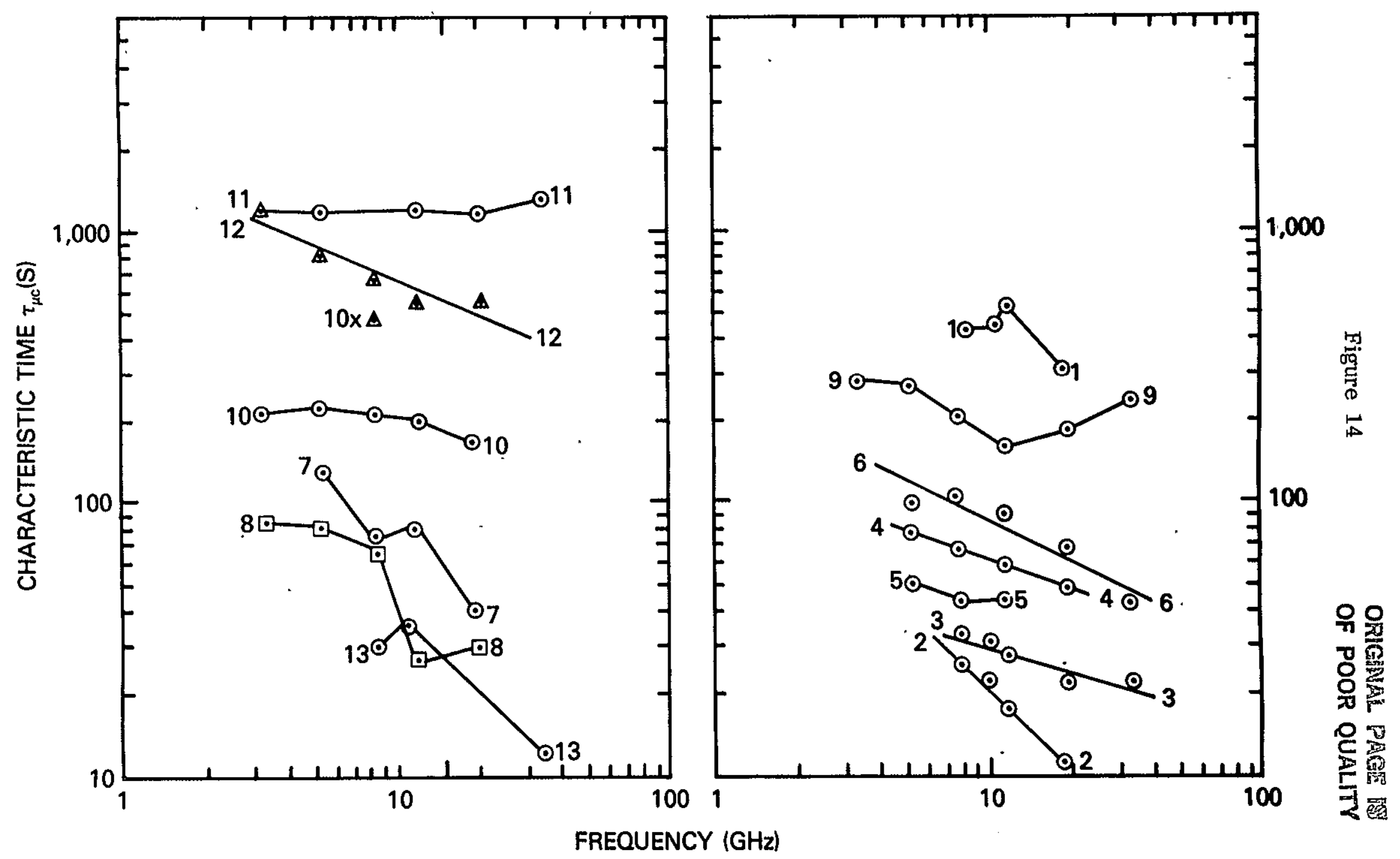




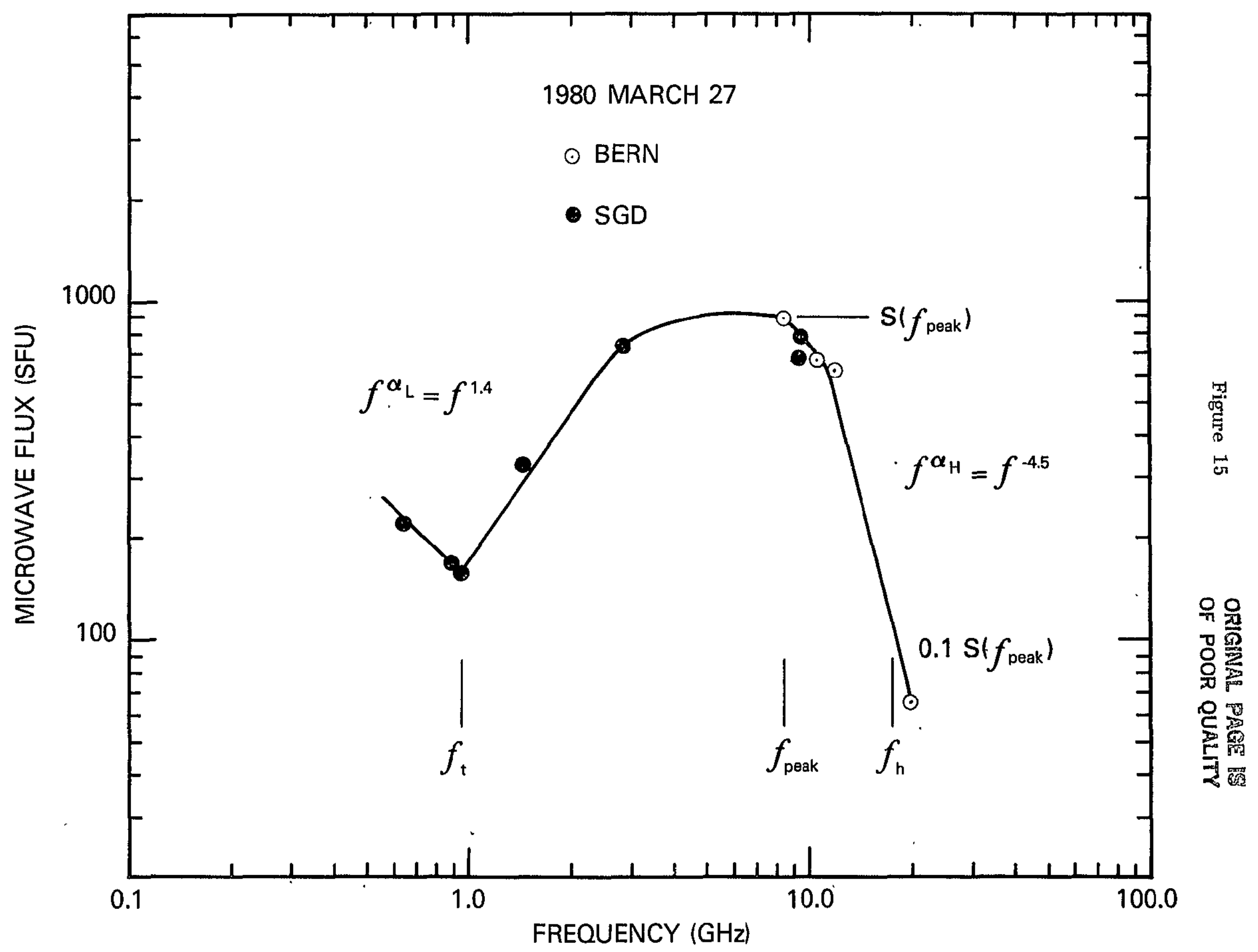




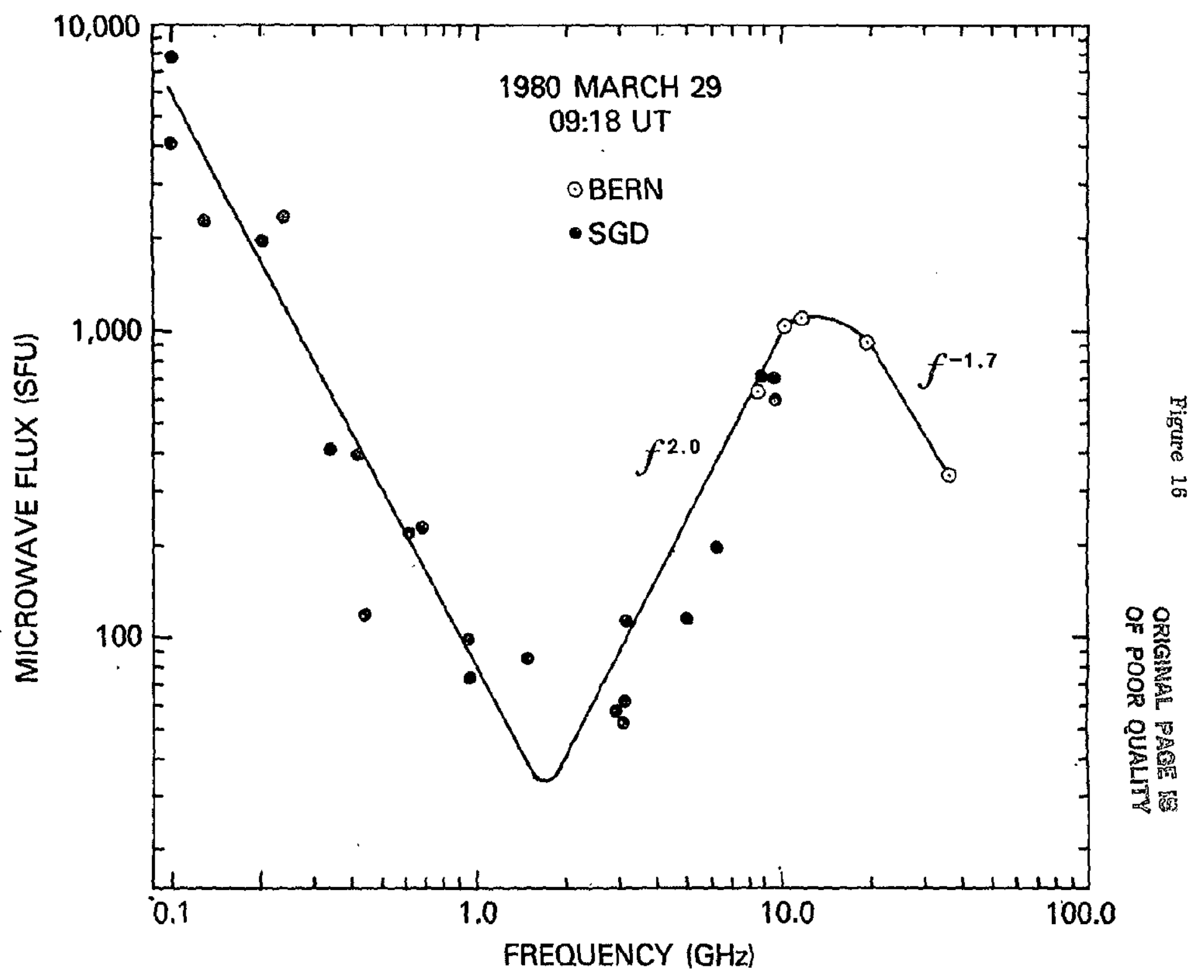




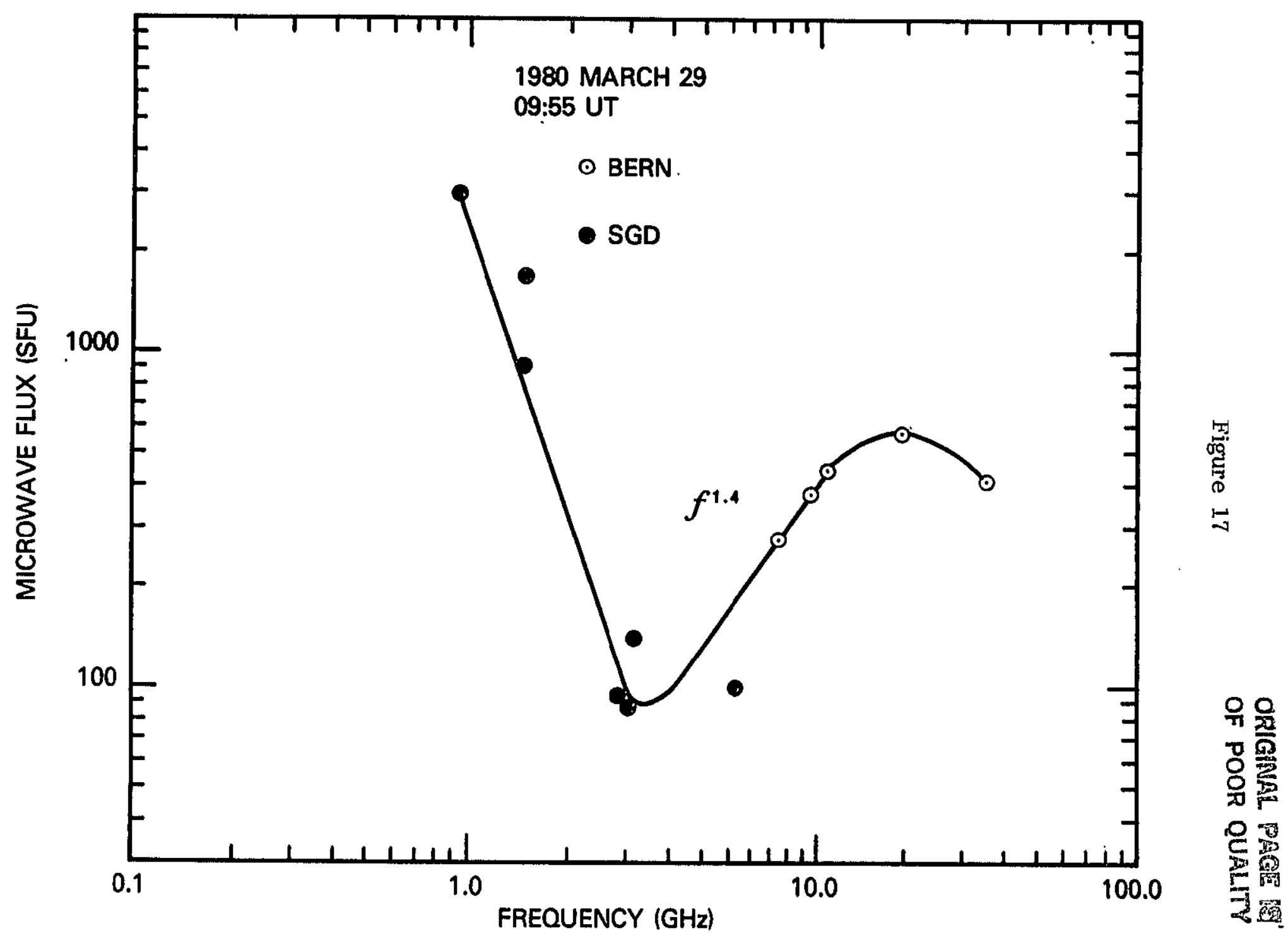

Fin 40000 pog 3 diak MCS.23 MASA BSAtaz EW 


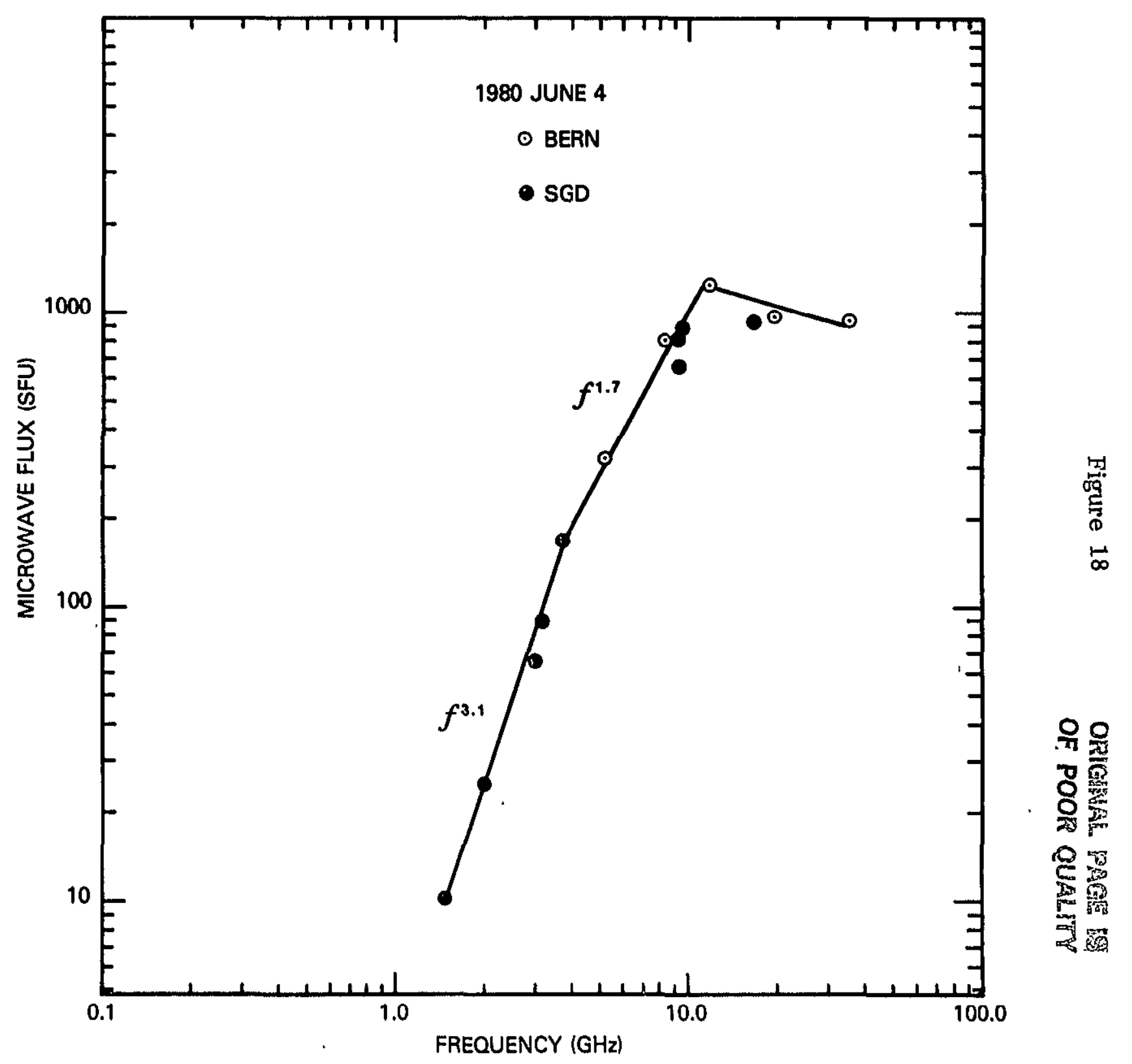




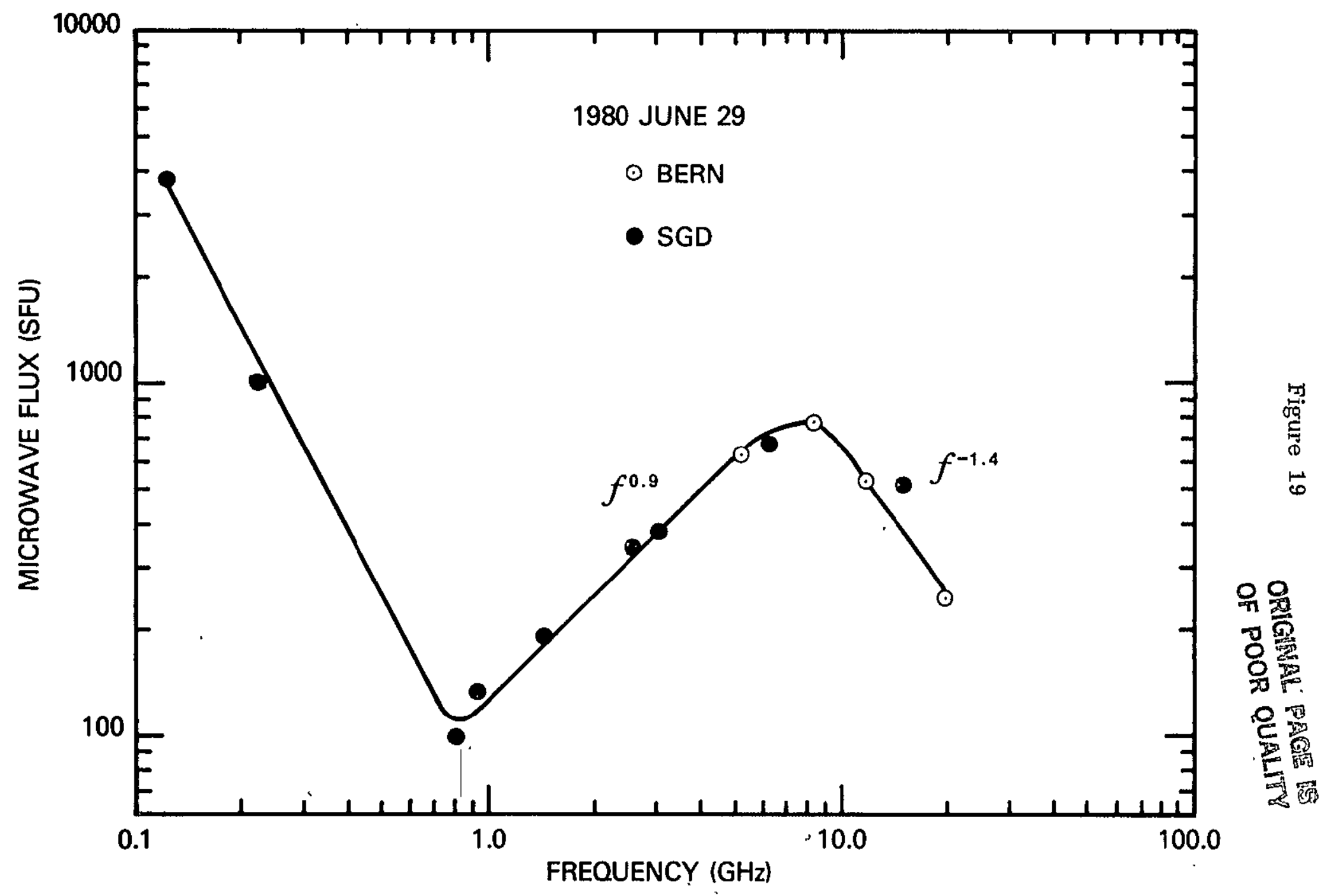

hie 46900 Do 2 das MCS 23 MASA 89 gR EW 


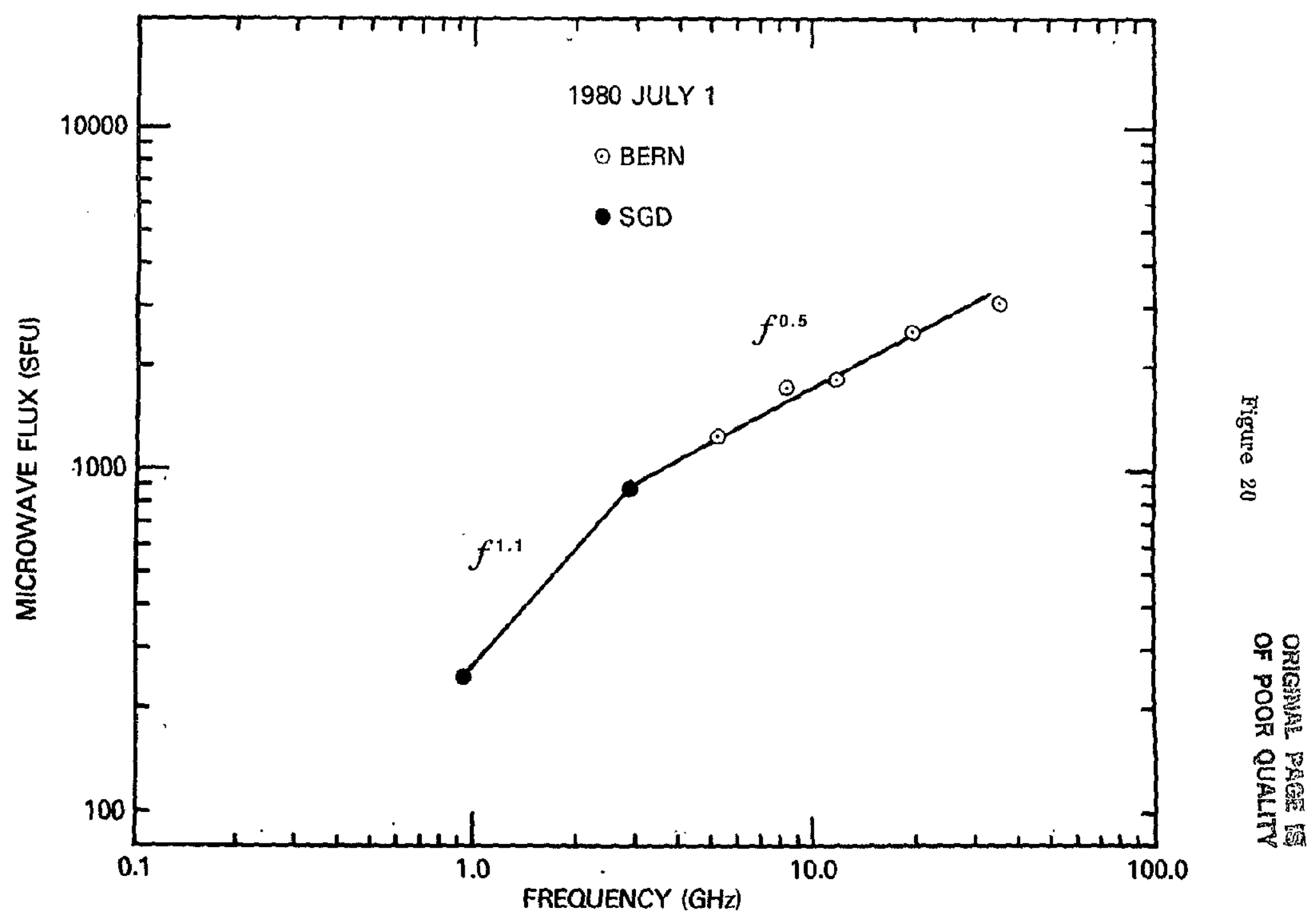


Figure 21

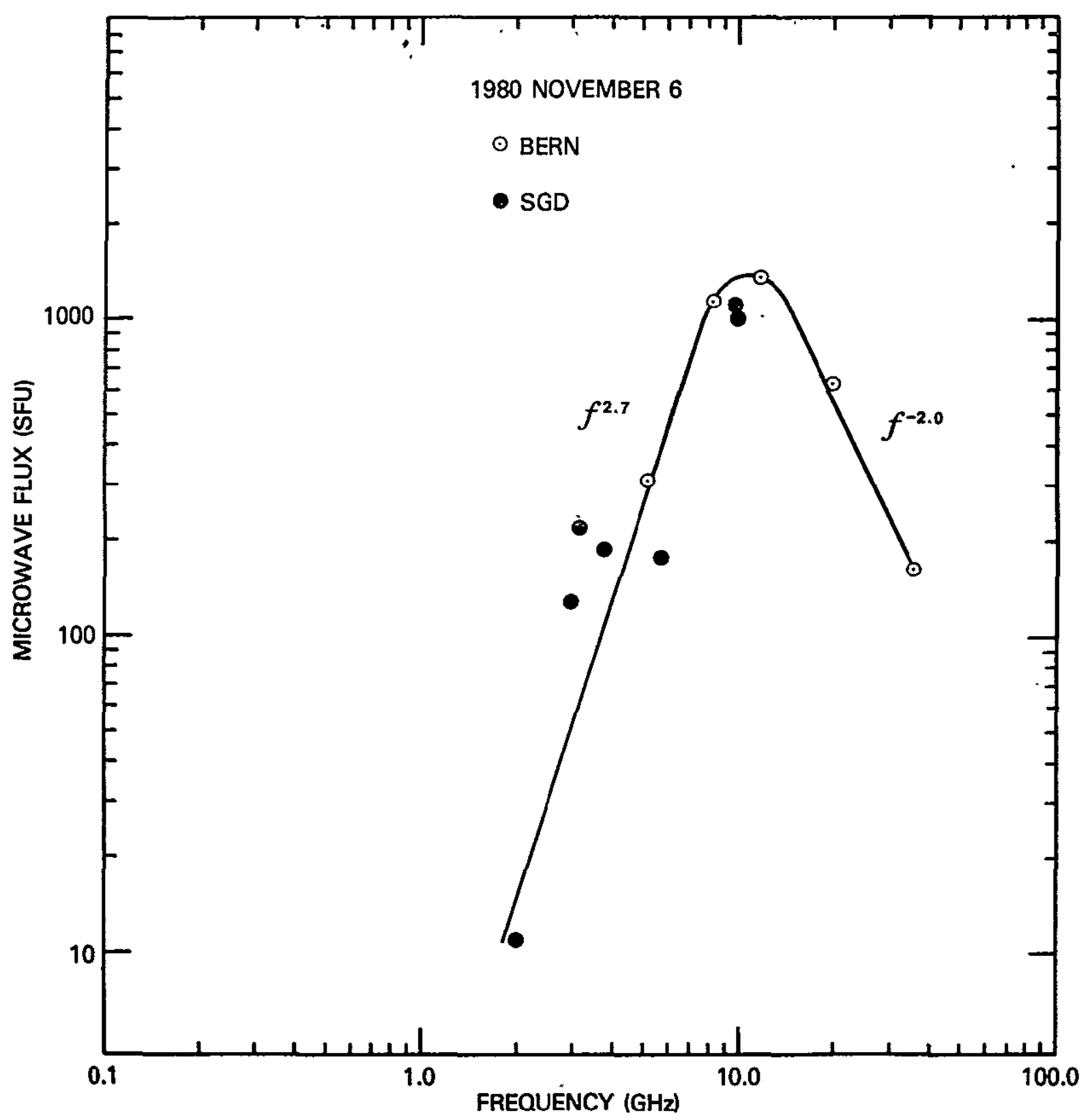




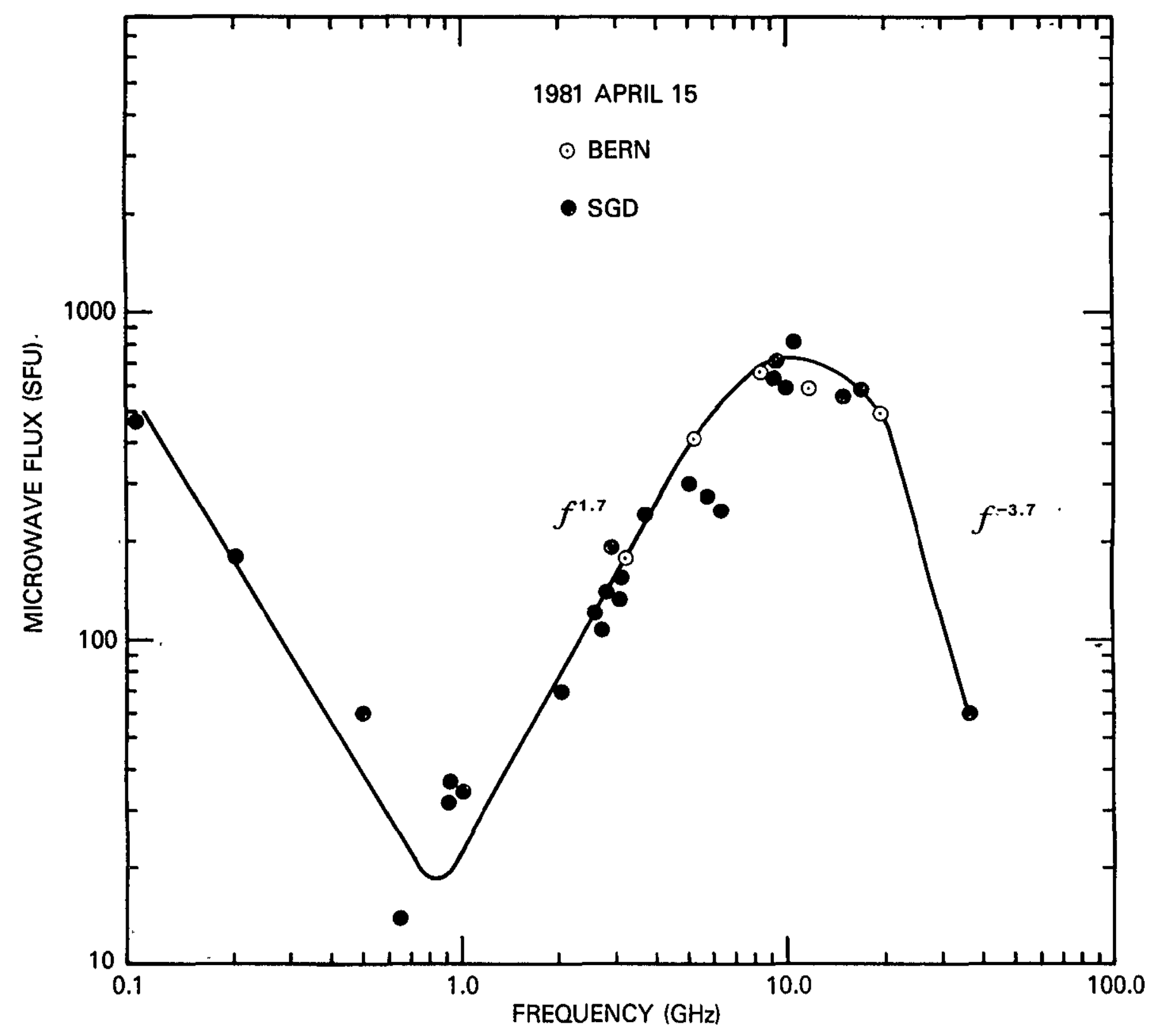

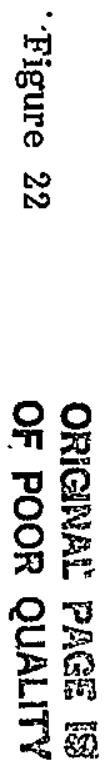




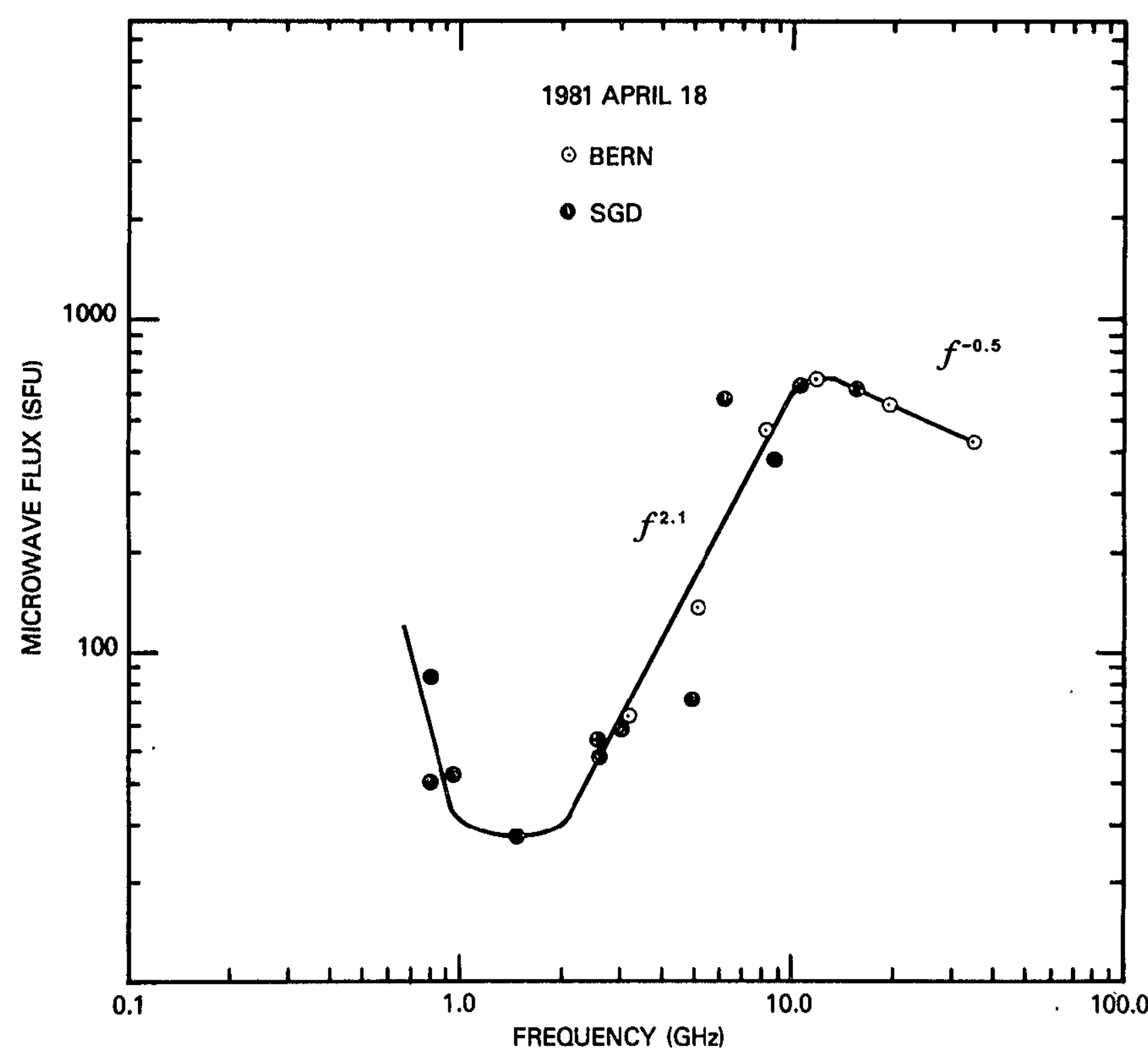

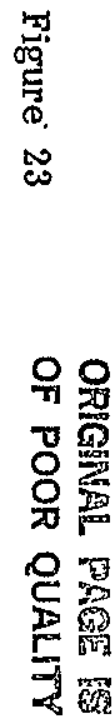

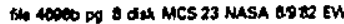




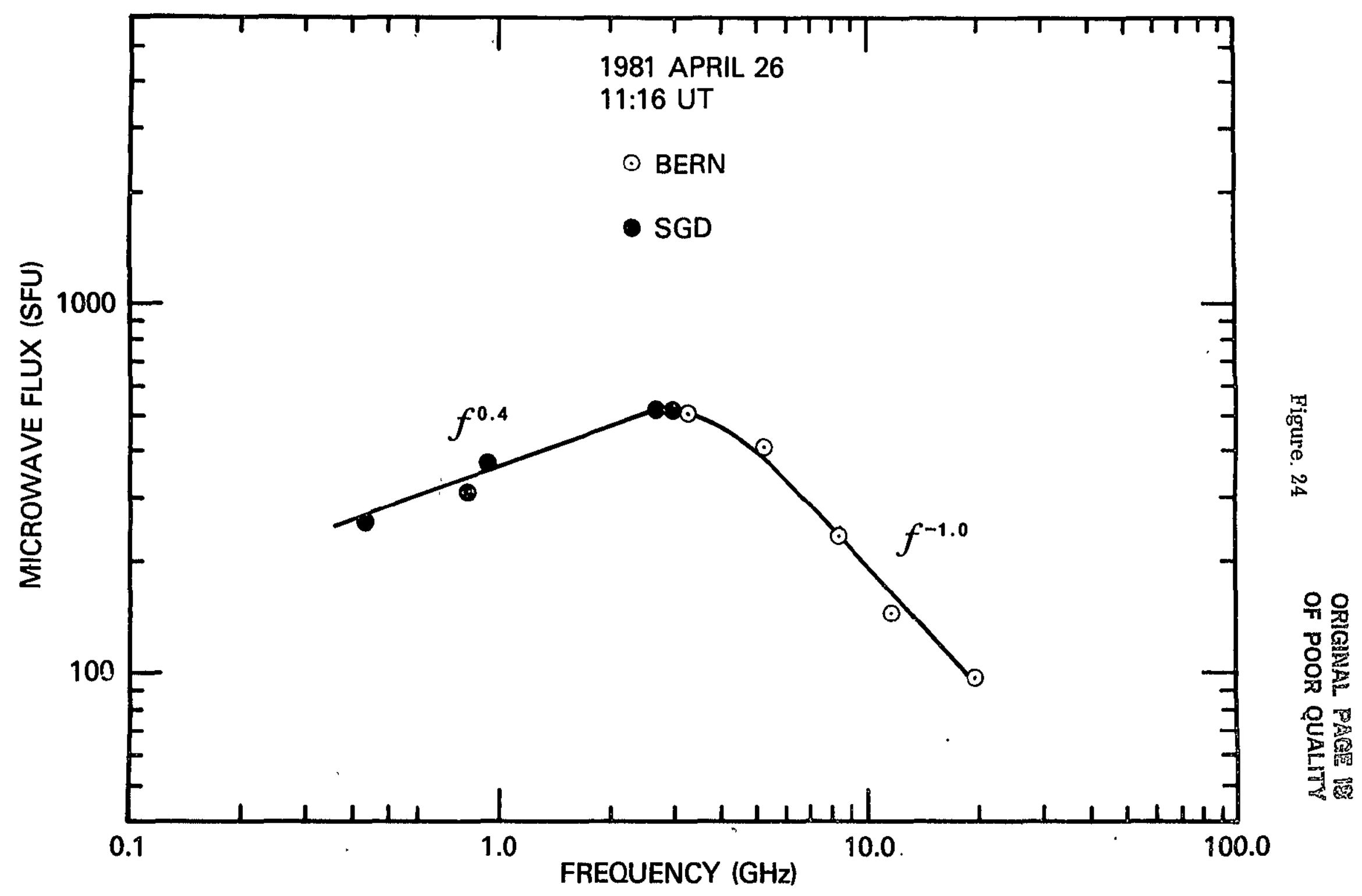




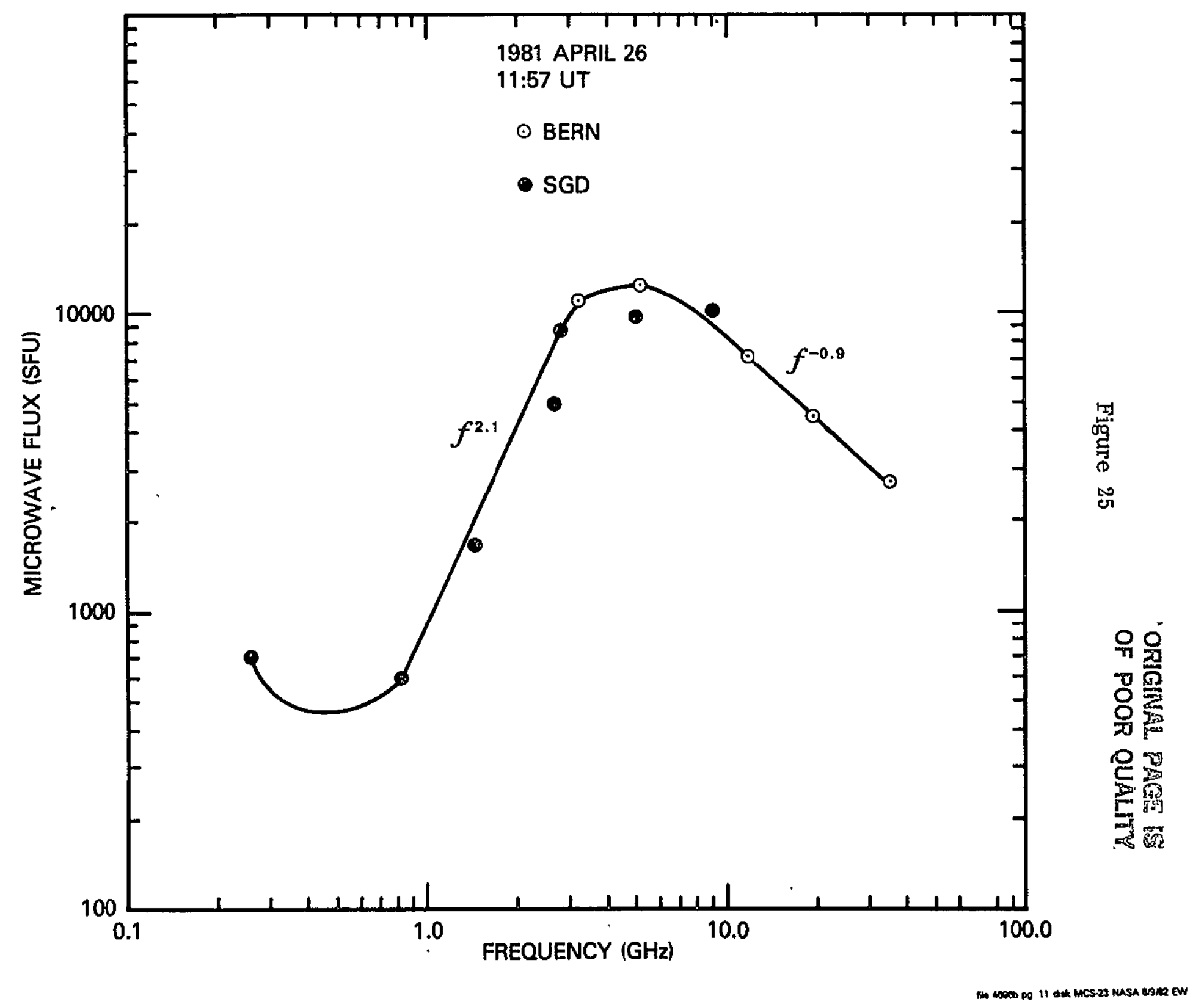




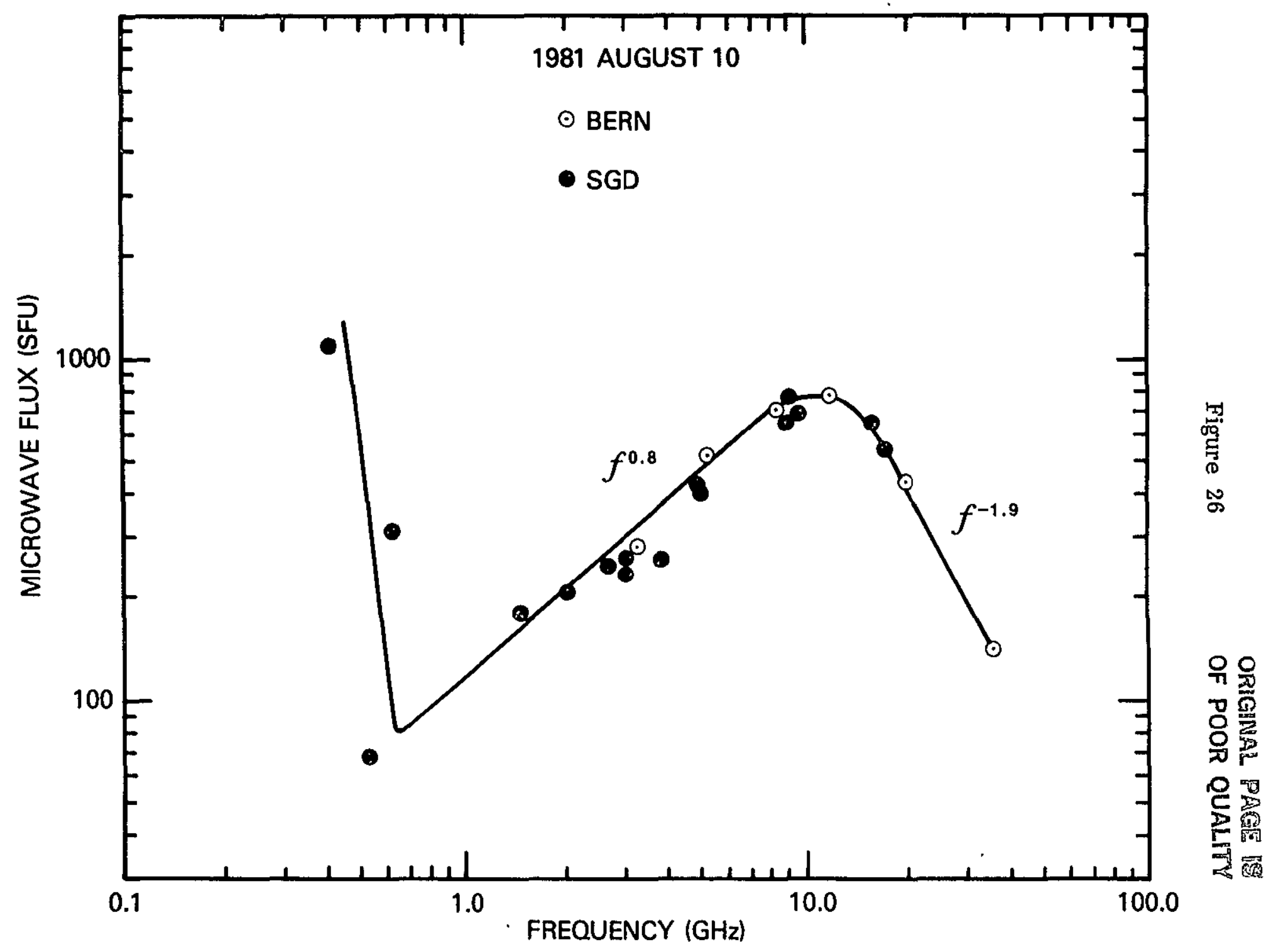

Fif 46980 pg 12 disk MCS 23 NASA B98 EW 


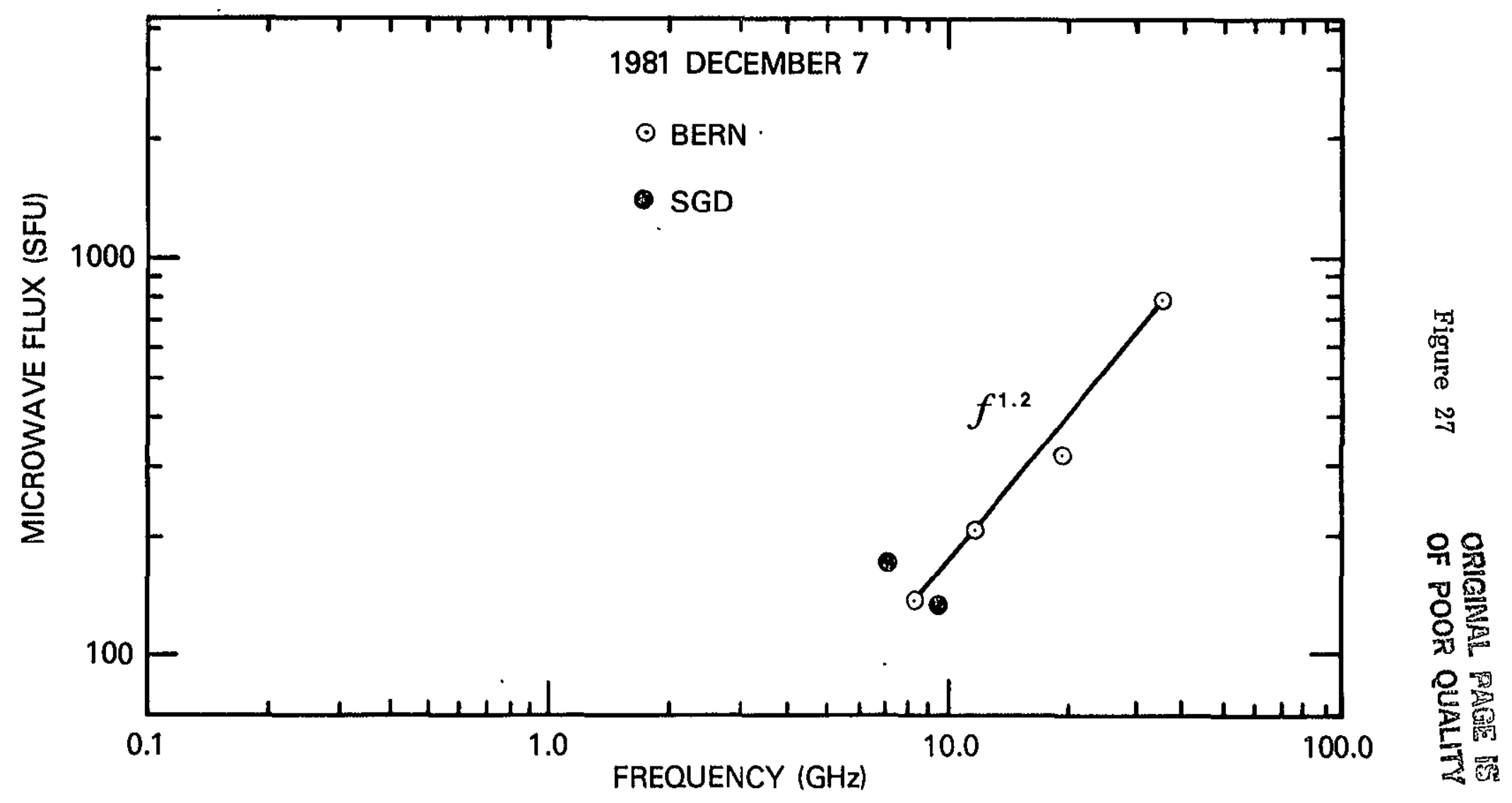




\section{MICROWAVE POLARIZATION}

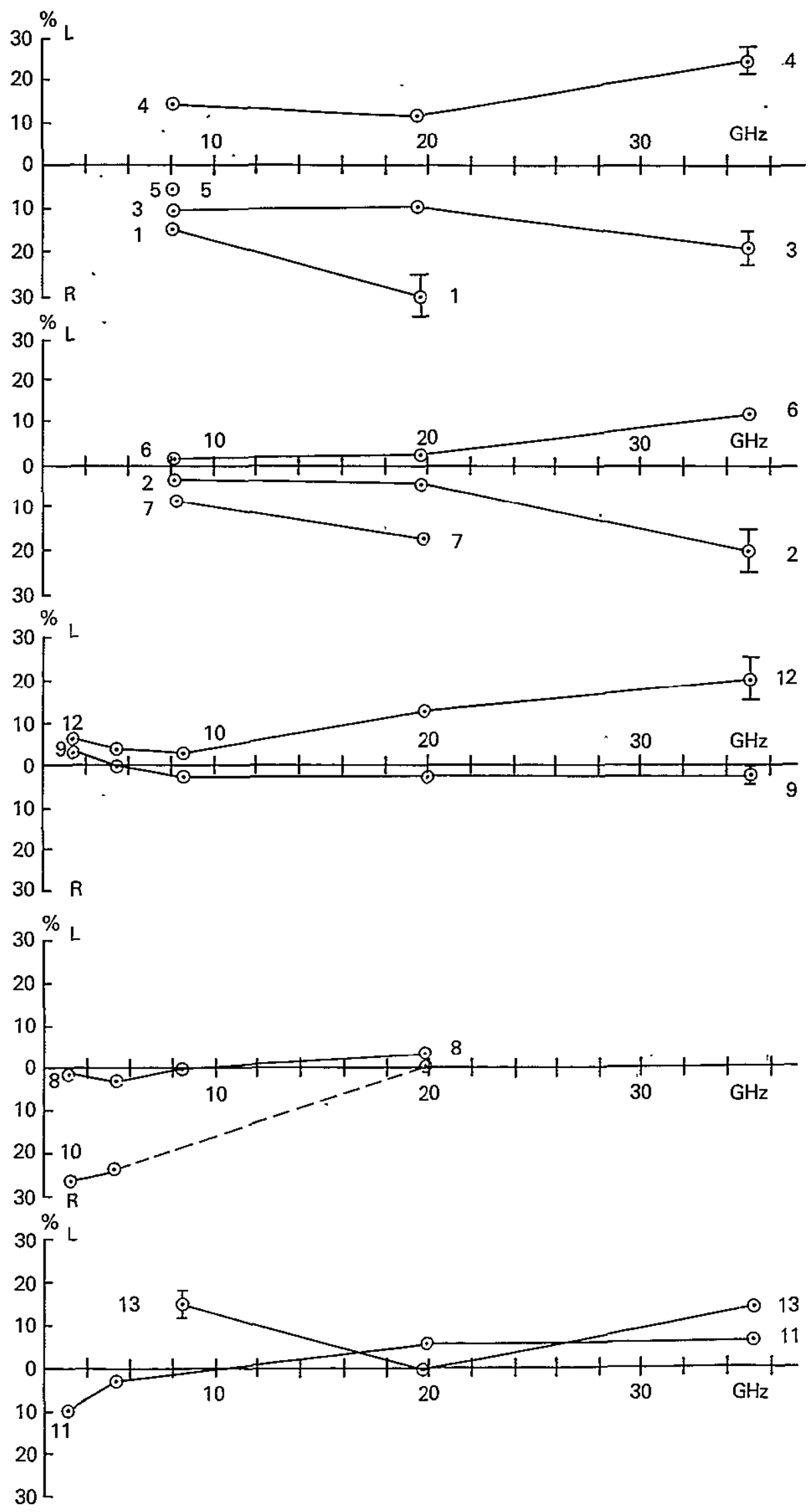




\section{HARD X-RAY CHARACTERISTIC TIMES}
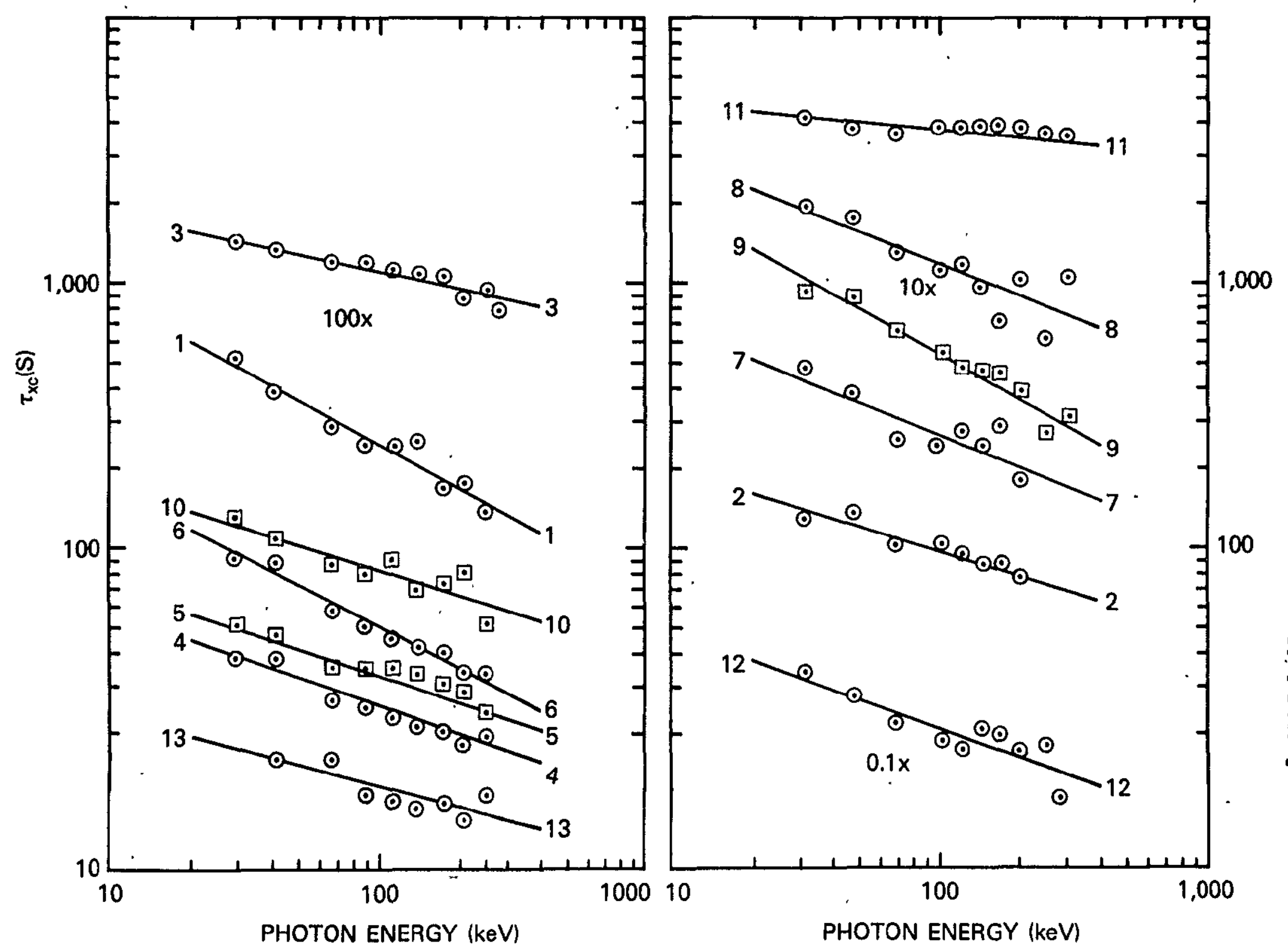


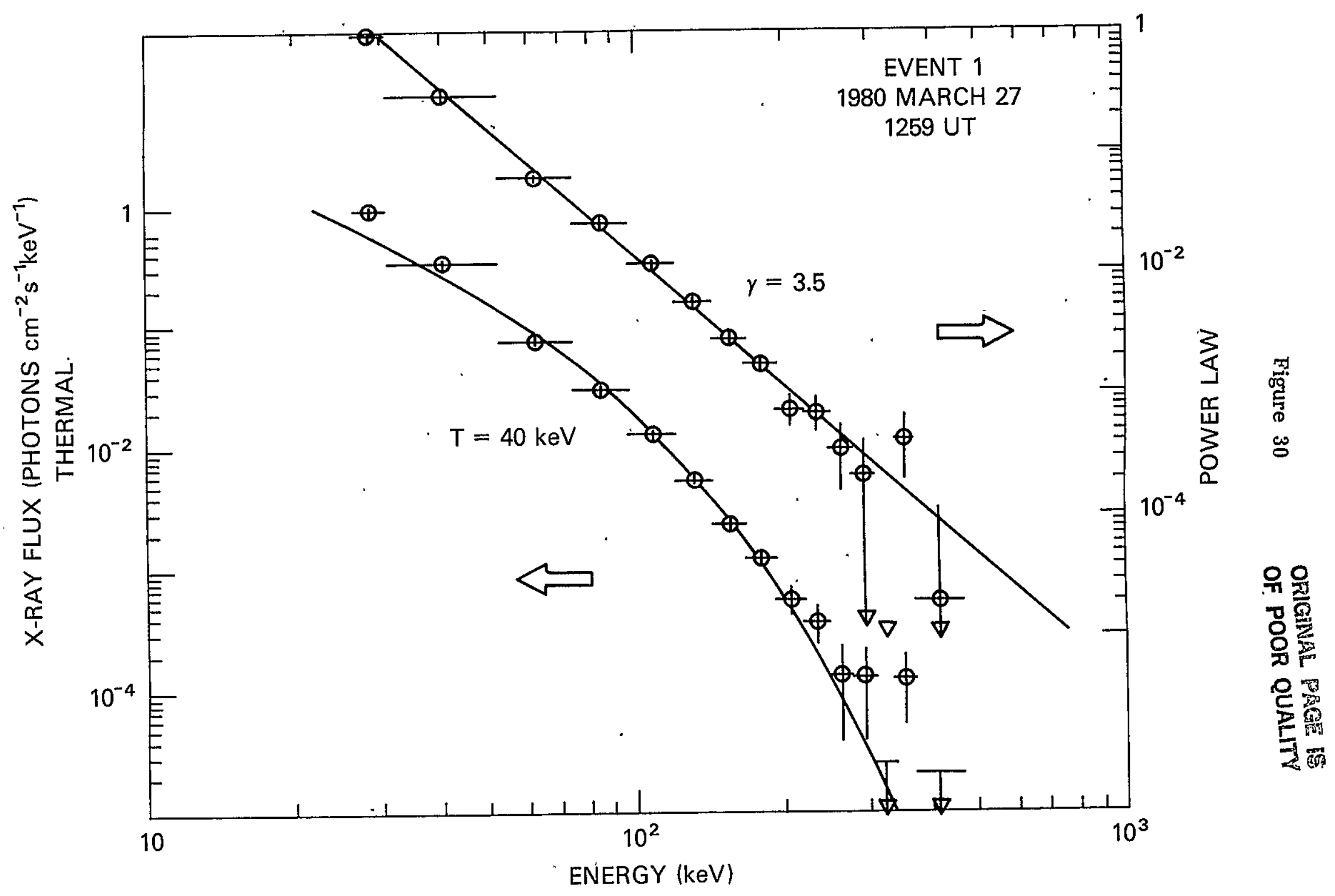




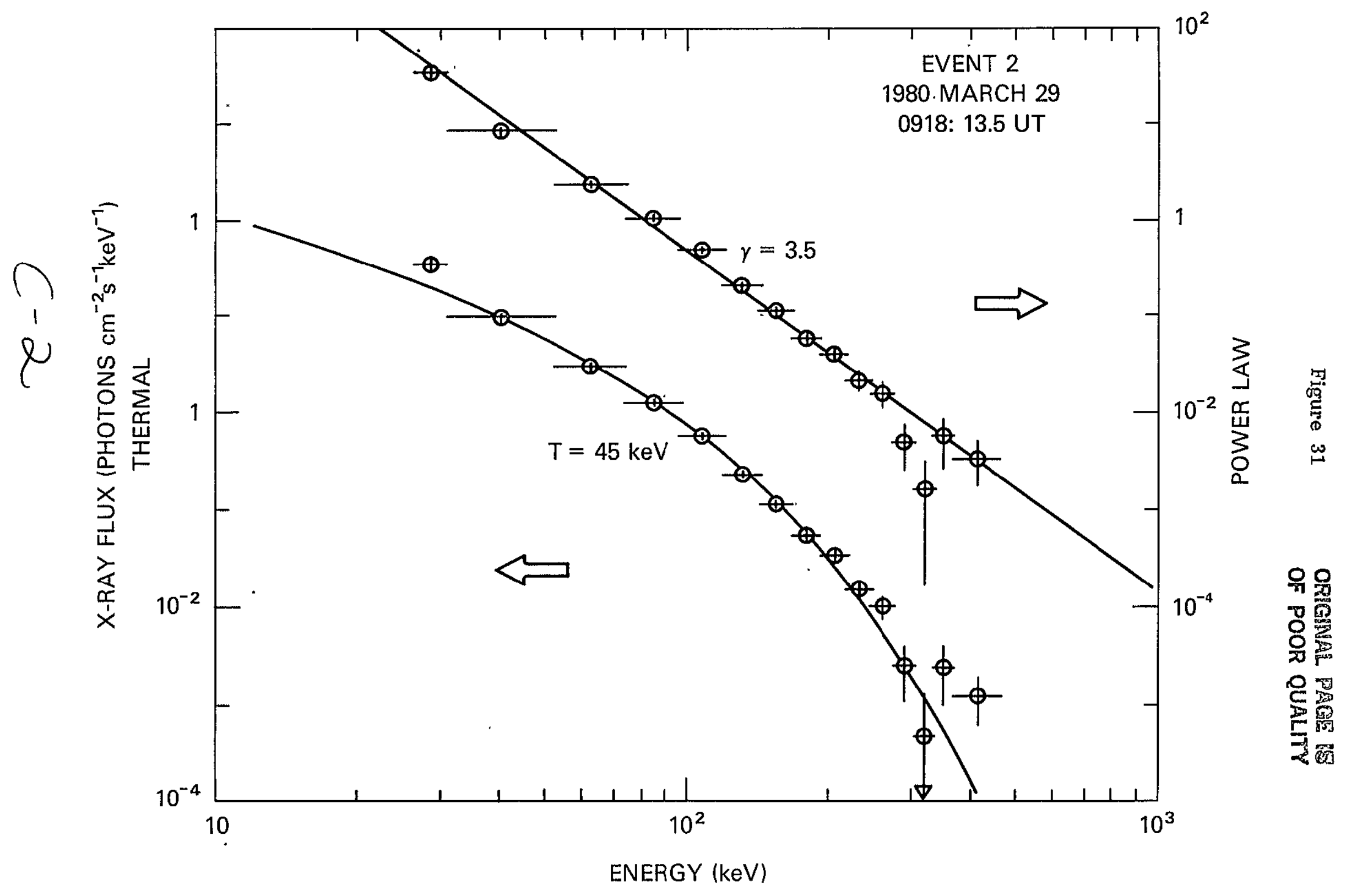




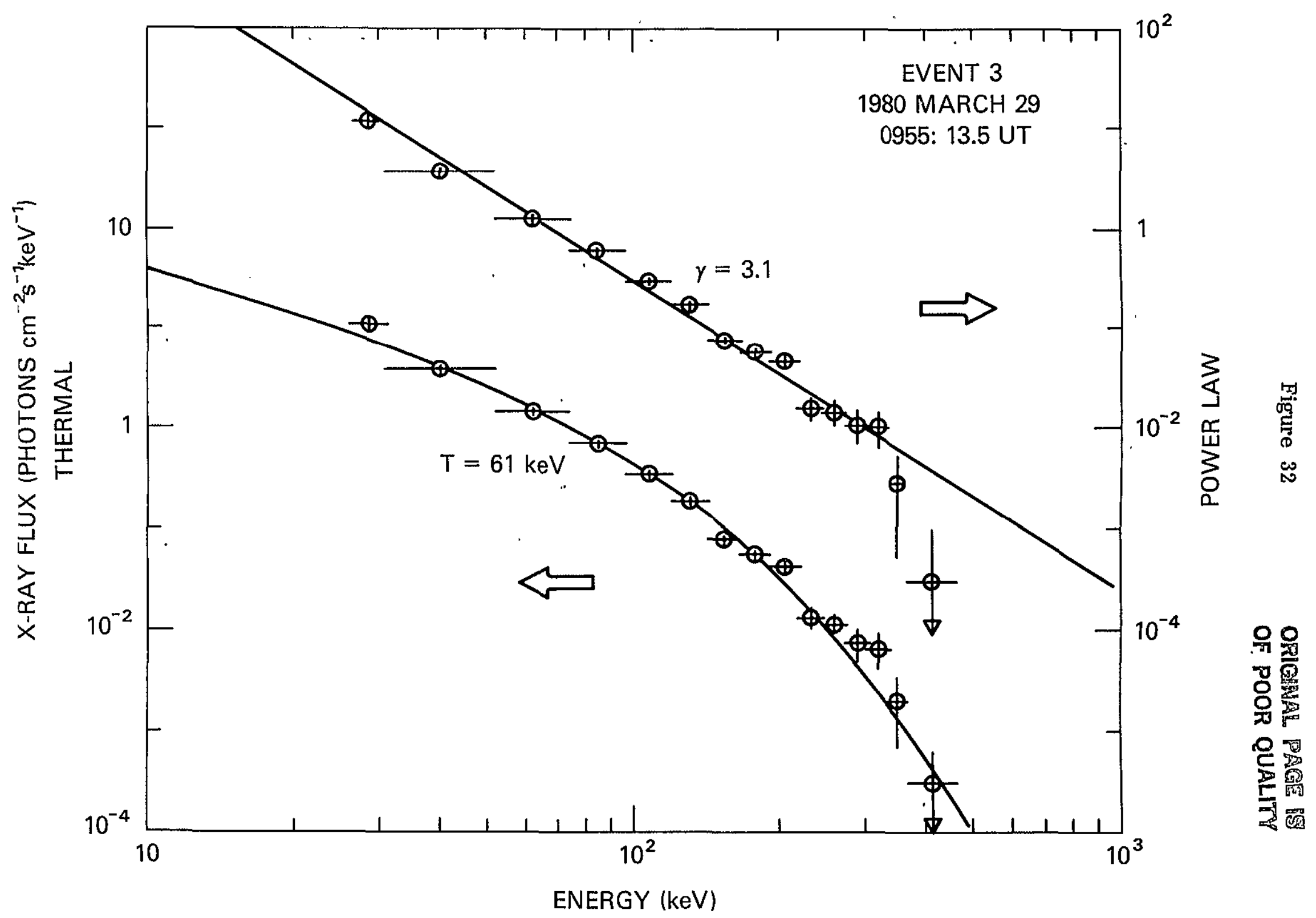




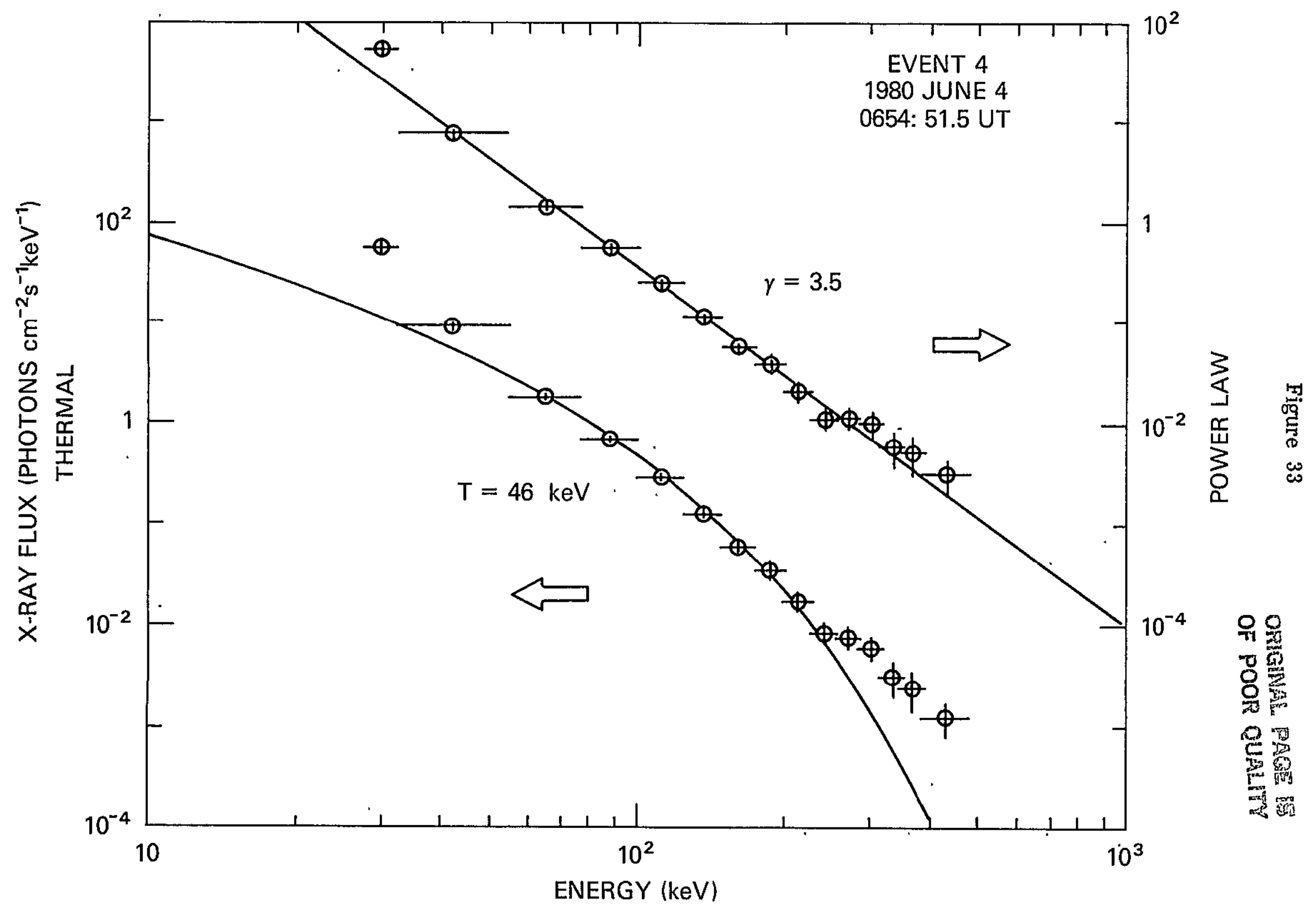




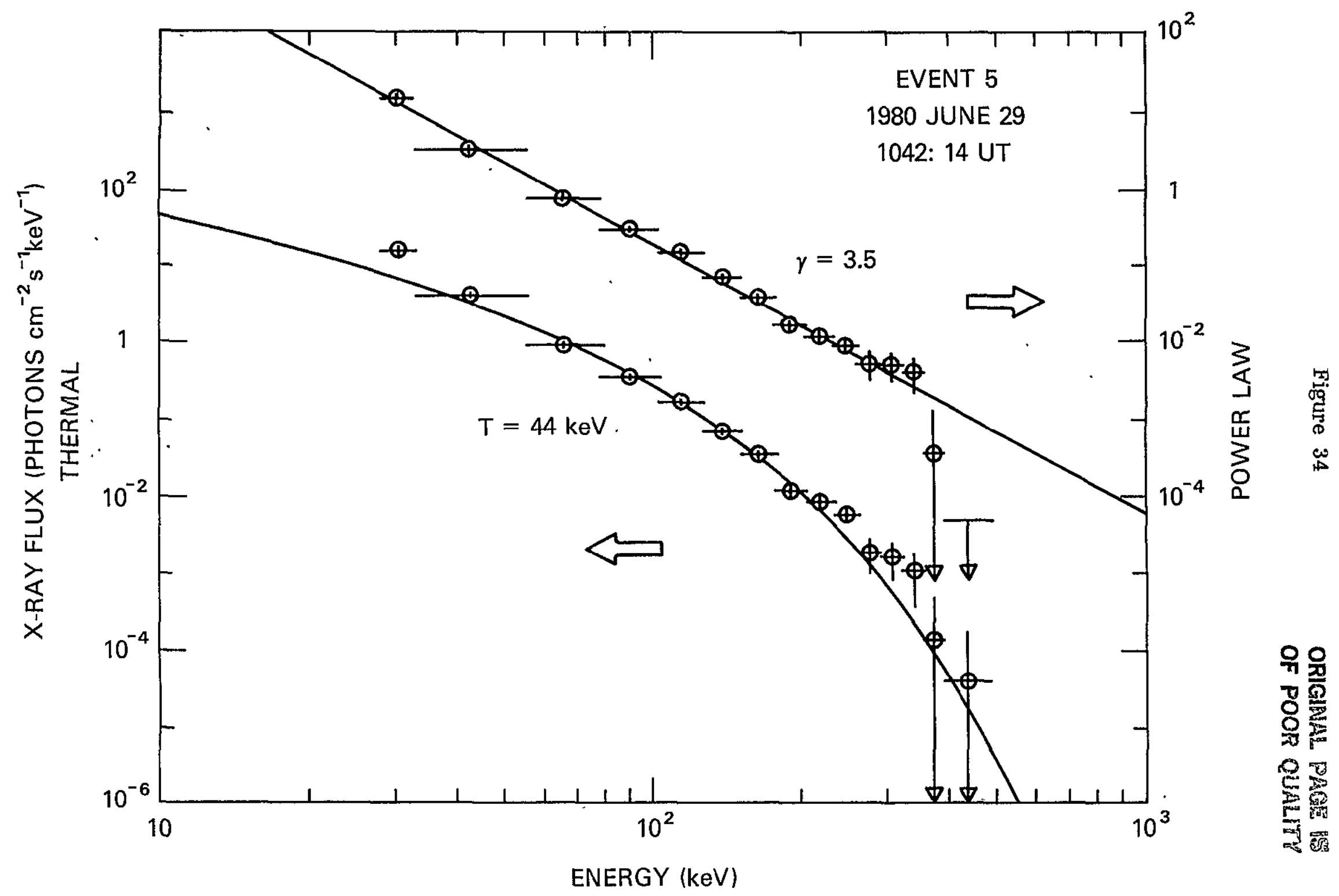




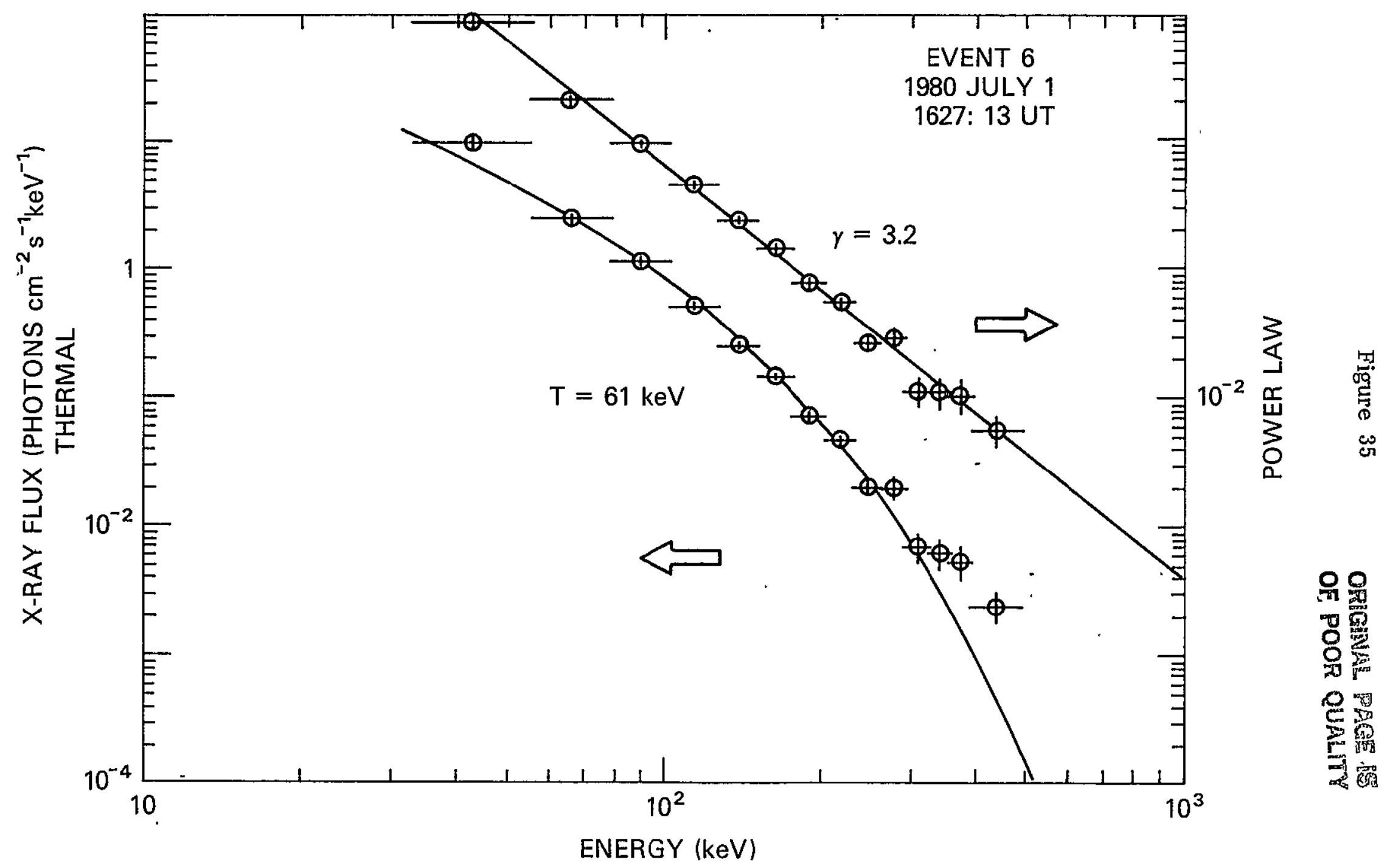




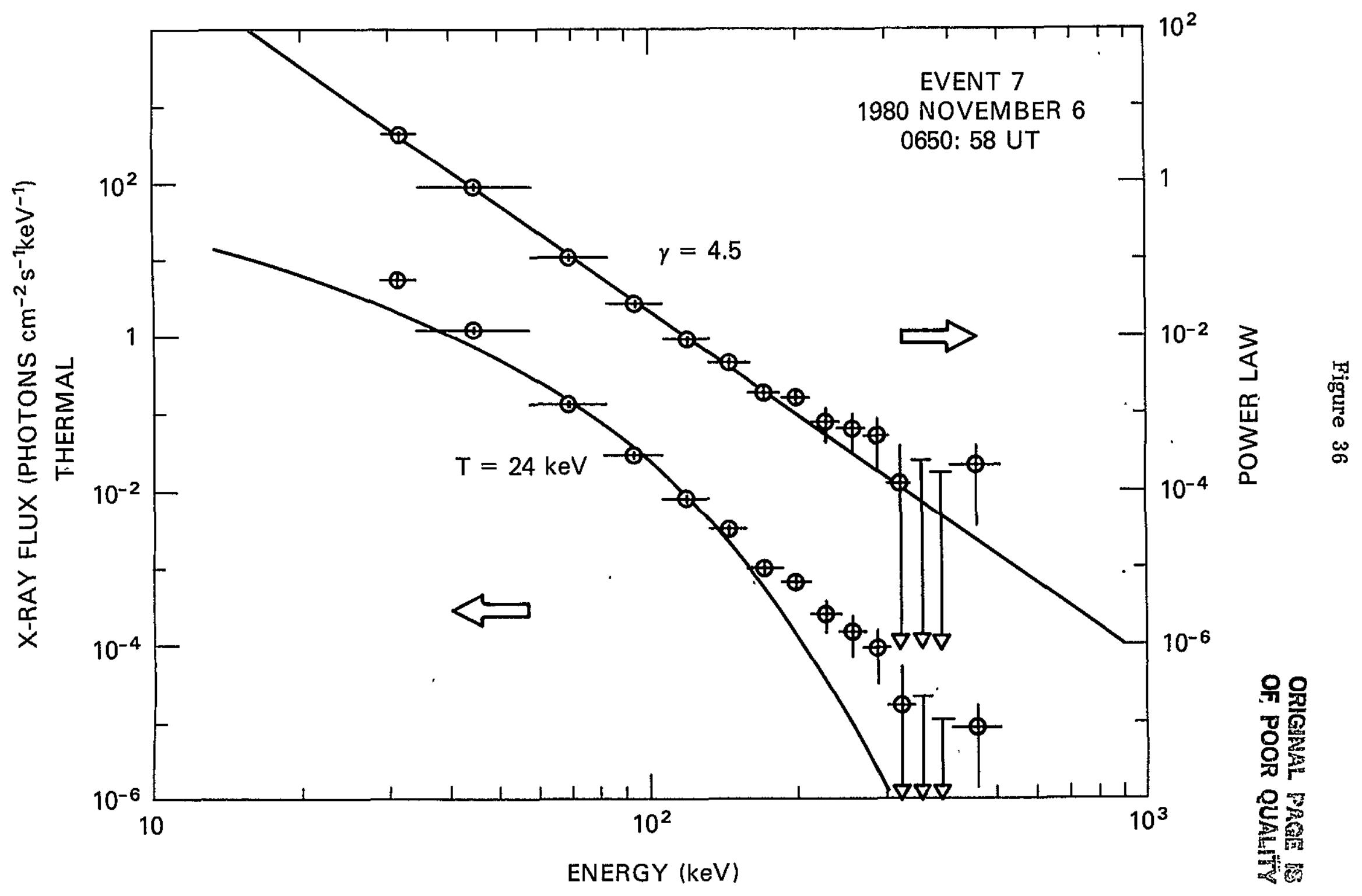




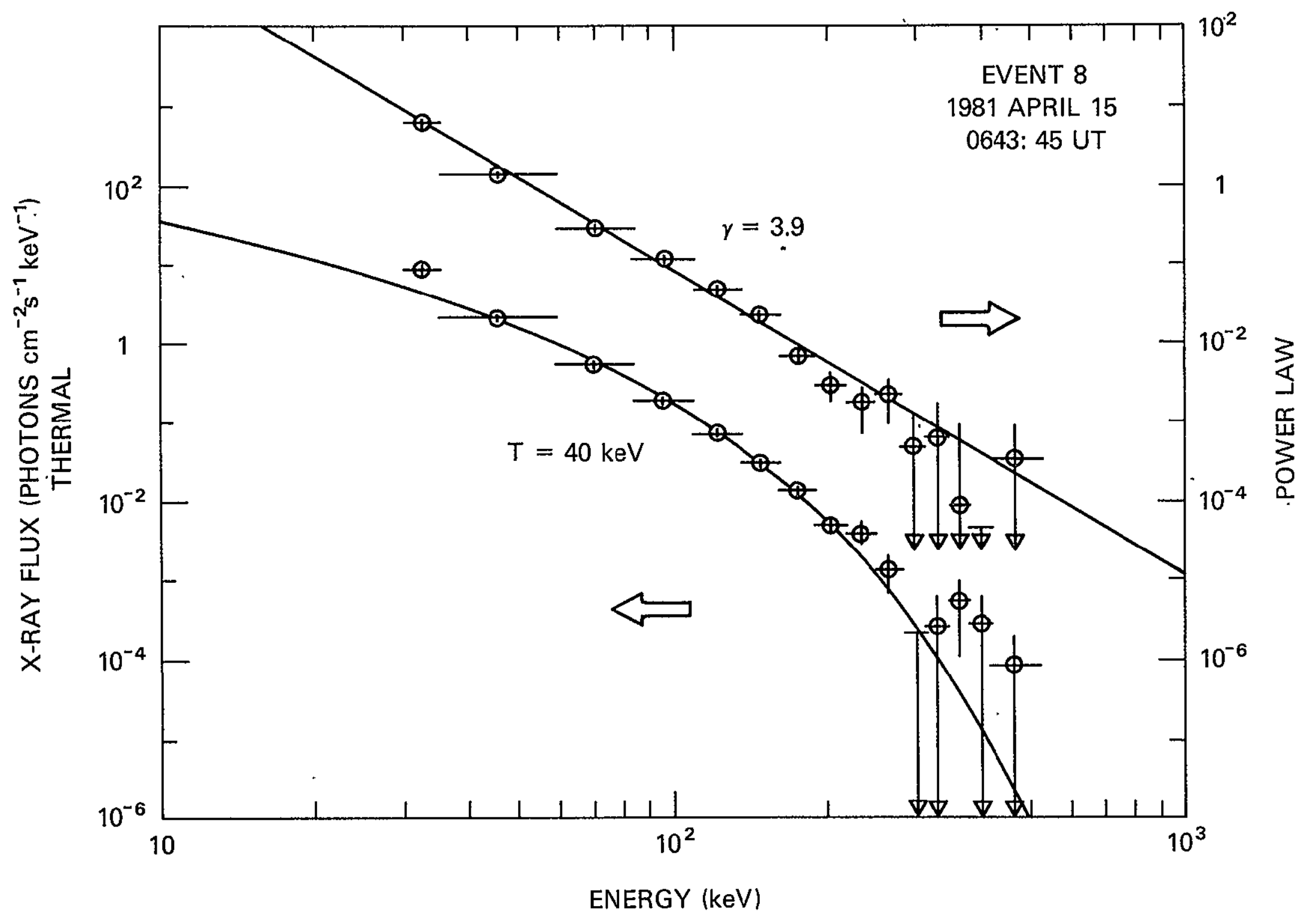

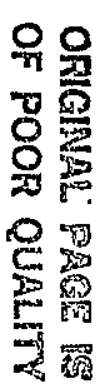




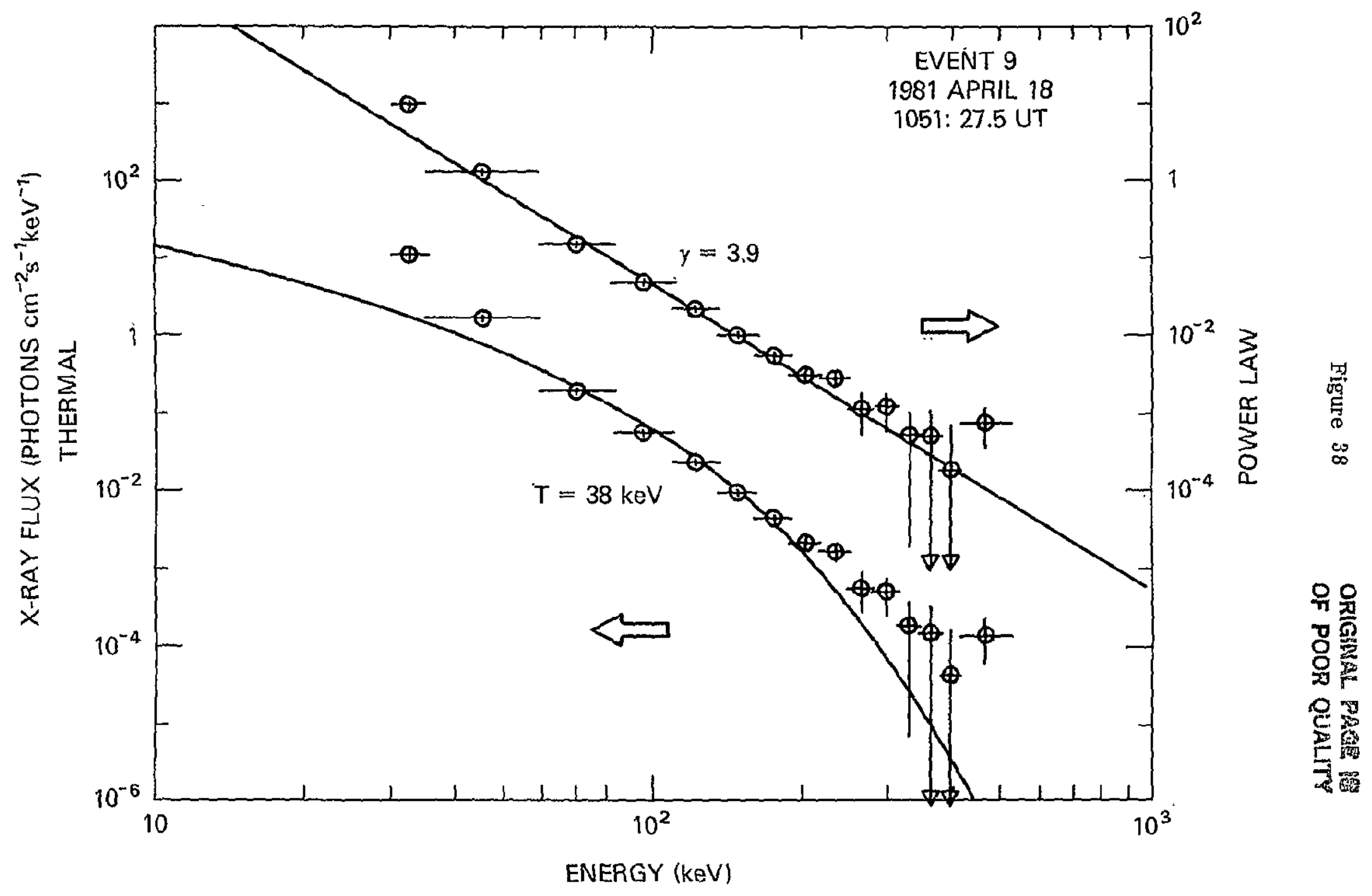




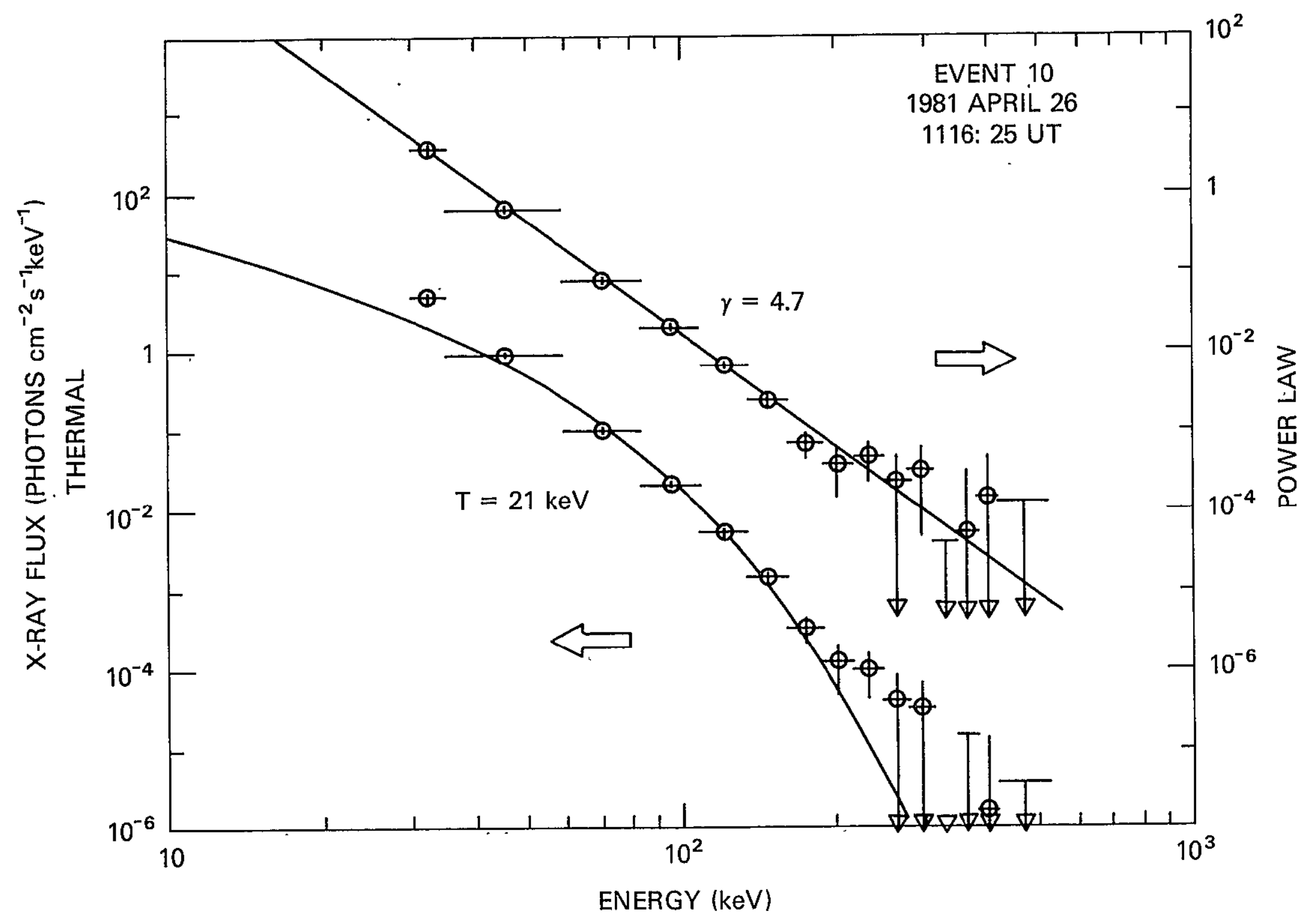




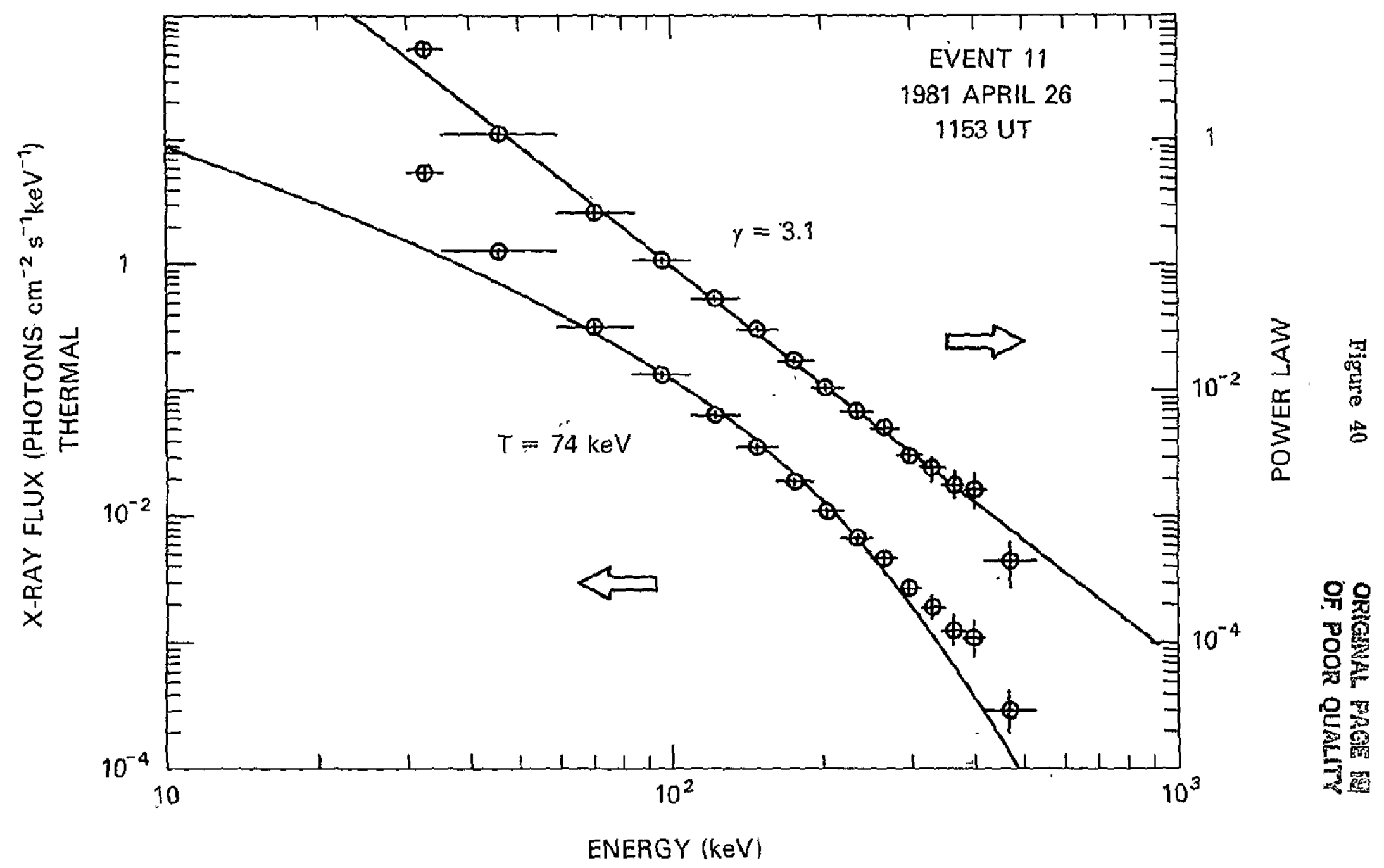




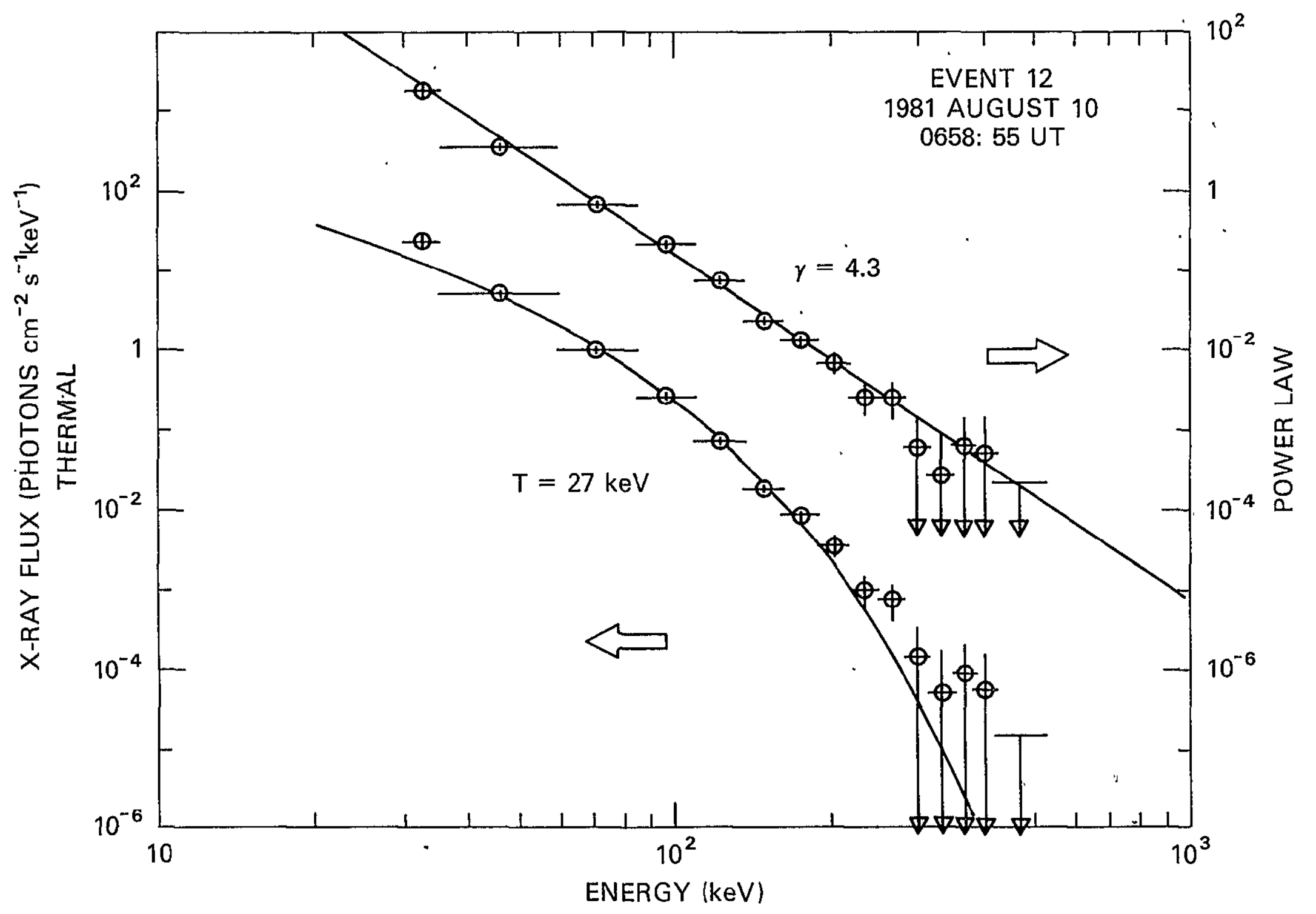

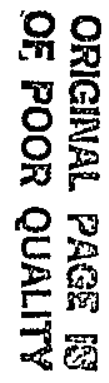




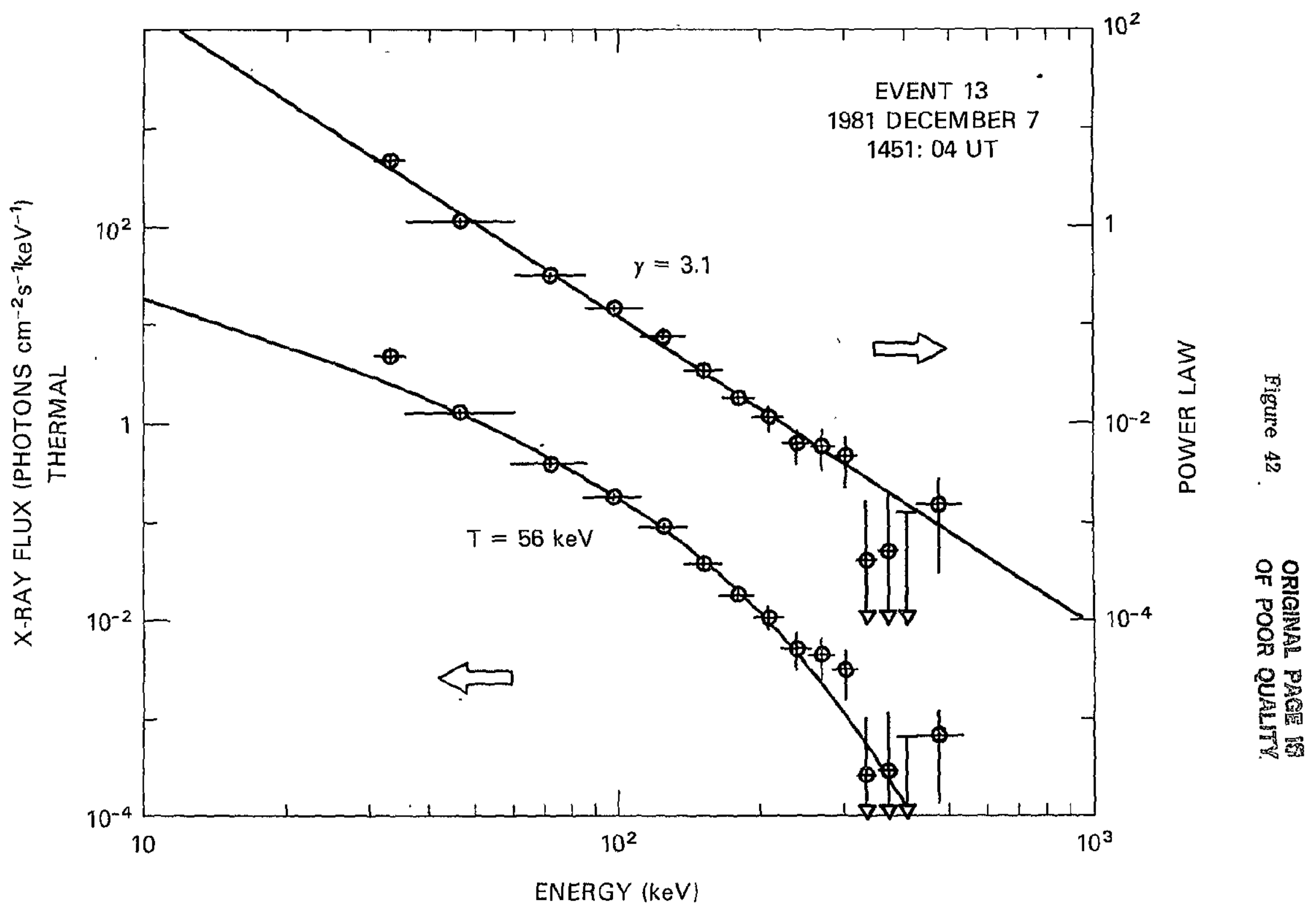



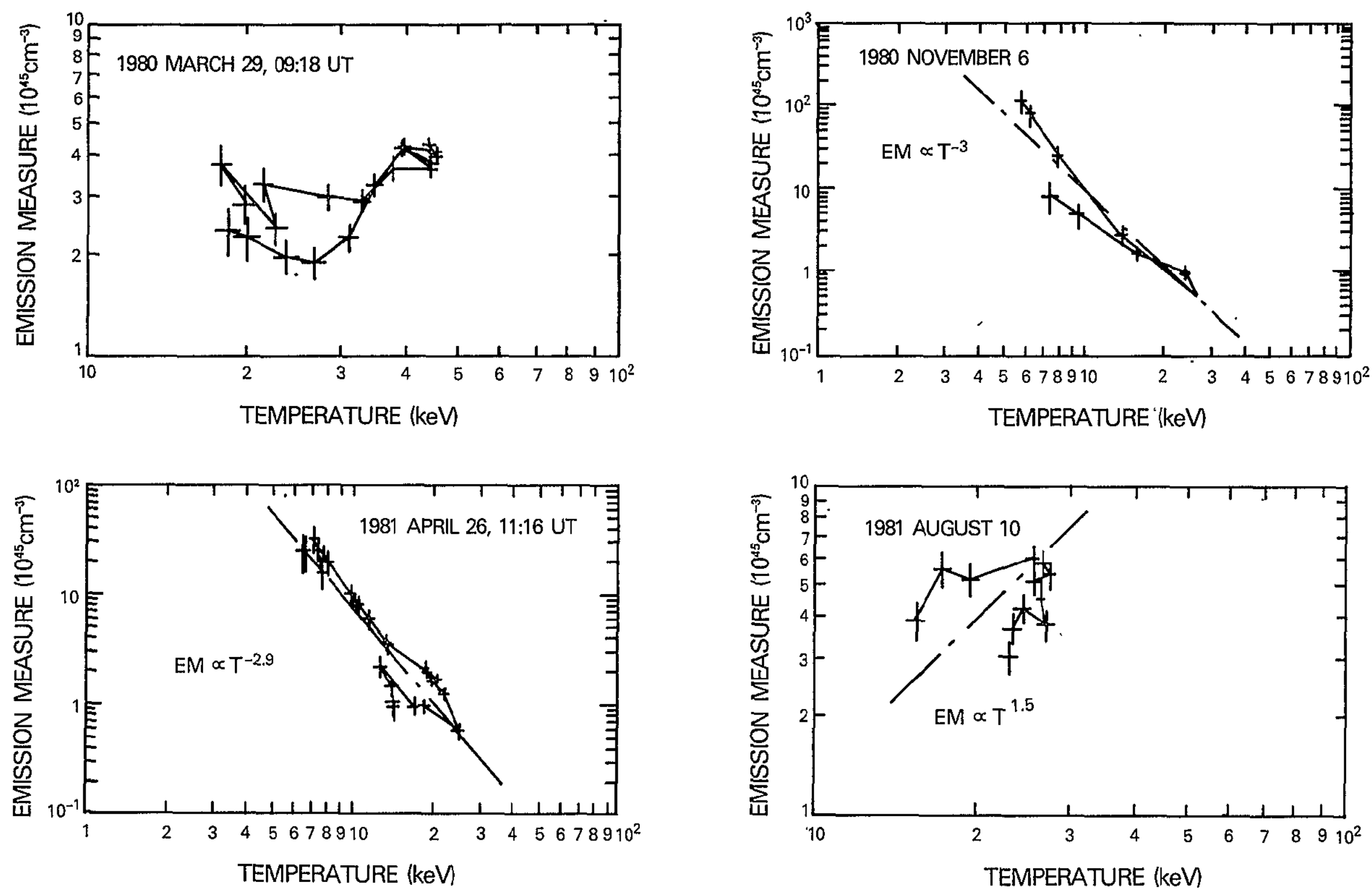

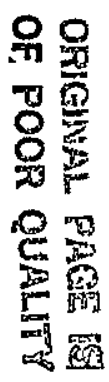




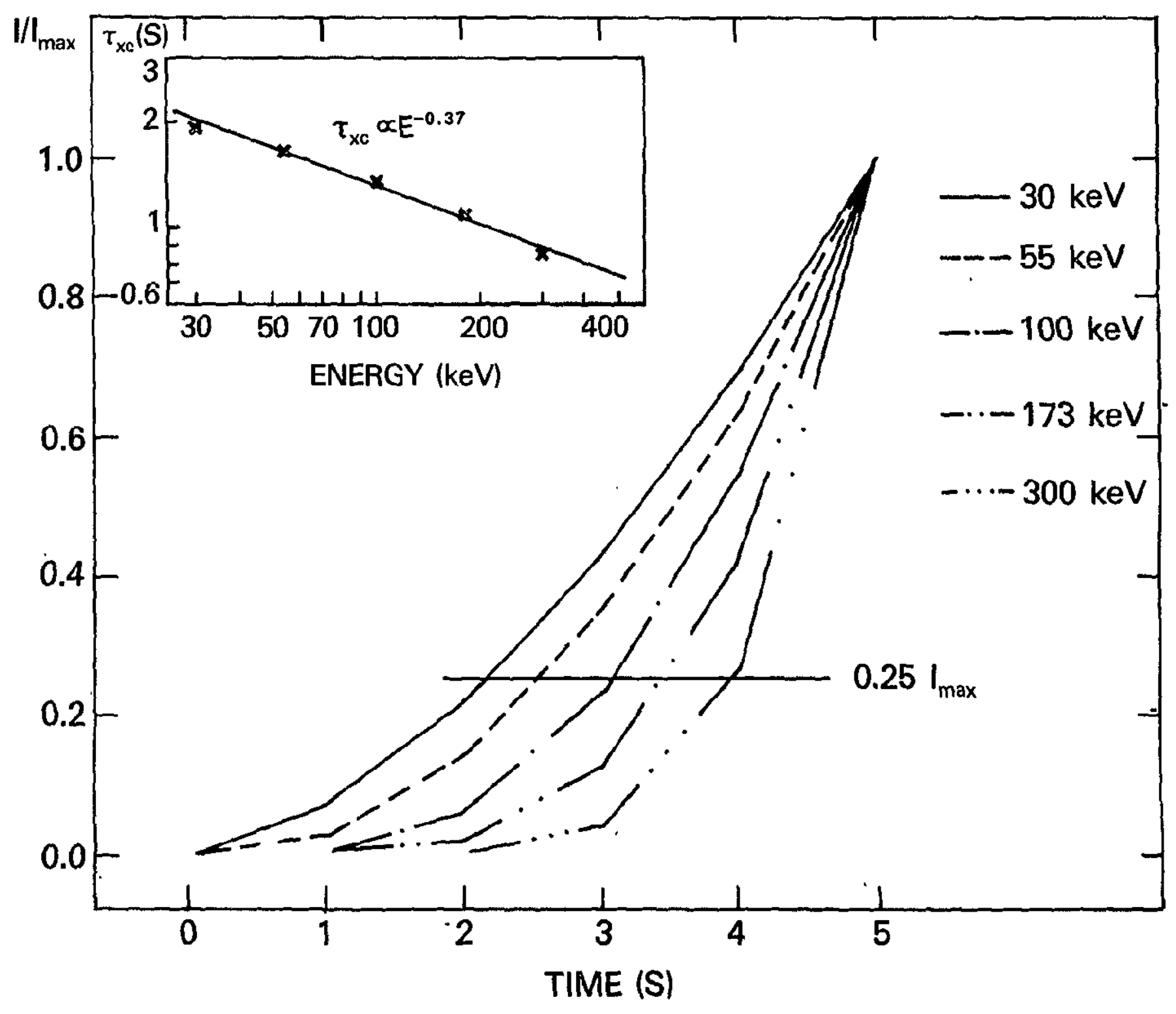

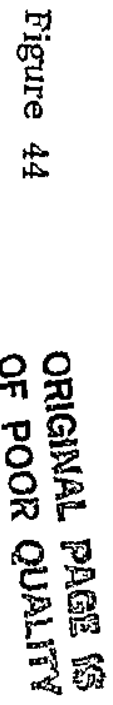




\section{BIBLIOGRAPHIC DATA SHEET}

\begin{tabular}{|c|c|}
\hline $\begin{array}{l}\text { 1. Report No. } \\
\text { TM } 85052\end{array}$ & 3. Recipient's Catalog No. \\
\hline 4. Title and Subtitle & 5. Report Date \\
\hline \multirow{2}{*}{$\begin{array}{l}\text { Great Microwave Bursts and Hard X Rays } \\
\text { from Solar Flares }\end{array}$} & June 1983 \\
\hline & $\begin{array}{l}\text { 6. Performing Organization Code } \\
684\end{array}$ \\
\hline $\begin{array}{l}\text { 7. Author(s) H. J. Wiehl, D. A. Batchelor, C. J. } \\
\text { Cranne11, B. R. Dennis and Phillip. N. Price }\end{array}$ & 8. Performing Organization Report No. \\
\hline \multirow{3}{*}{$\begin{array}{l}\text { 9. Performing Organization Name and Address } \\
\text { NASA-Goddard Space. Flight Center } \\
\text { Greenbelt, Maryland } 20771\end{array}$} & 10. Work Unit No. \\
\hline & 11. Contract or Grant No. \\
\hline & 13. Type of Report and Period Covered \\
\hline \multirow[t]{2}{*}{ 12. Sponsoring Agency Name and Address } & \\
\hline & 14. Sponsoring Agency Code \\
\hline
\end{tabular}

15. Supplementary Notes

16. Abstract.

The micröwave and hard X-ray characteristics of 13 solar flares that produced microwave fluxes greater than 500 Solar Flux Units have been analyzed. Thes Great Microwave Bursts were observed in the frequency range from 3 to $35 \mathrm{GHz}$ at Berne, and simultaneous hard $X$-ray observations were made in the energy range from 30 to $500 \mathrm{keV}$ with the Hard X-Ray Burst Spectrometer on the Solar Maximum Mission spacecraft. The principal aim of this analysis is to determine whether or not the same distribution of energetic electrons can explain both emissions. Correlations were found between respective temporal characteristics and, for the first time, between microwave and hard X-ray.spectral characteristics. A single-temperature and a multi-temperature model from the literature were tested for consistency with the coincident $X$-ray and microwave spectra at microwave burst maximum. Four events are inconsistent with both of the models tested, and neither of the models attempts to explain the high-frequency part of the microwave spectrum. We propose a model in which the emissions above and below the peak frequency originate in two different parts of a diverging magnetic loop. With this model we explain the entire microwave spectrum of all but 1 of the events.

17. Key Words (Selected by Author(s))

Plasmas - Sun: Bursts - Sun:

Microwaves - Sun: X-Rays
18. Distribution Statement

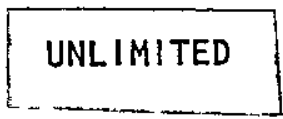

19. Security Classif. (of this report) UNCLASSIFIED

\section{Security Classif. (of this page) UNCLASSIFIED}

21. No. of Pages

22. Price $^{*}$ 